\title{
WestVirginiaUniversity
}

THE RESEARCH REPOSITORY @ WVU

Graduate Theses, Dissertations, and Problem Reports

2014

\section{Assessment strategies for mixing in very viscous gas-liquid two- phase flows}

Sharad Chand Ravinuthala

West Virginia University

Follow this and additional works at: https://researchrepository.wvu.edu/etd

\section{Recommended Citation}

Ravinuthala, Sharad Chand, "Assessment strategies for mixing in very viscous gas-liquid two-phase flows" (2014). Graduate Theses, Dissertations, and Problem Reports. 395.

https://researchrepository.wvu.edu/etd/395

This Thesis is protected by copyright and/or related rights. It has been brought to you by the The Research Repository @ WVU with permission from the rights-holder(s). You are free to use this Thesis in any way that is permitted by the copyright and related rights legislation that applies to your use. For other uses you must obtain permission from the rights-holder(s) directly, unless additional rights are indicated by a Creative Commons license in the record and/ or on the work itself. This Thesis has been accepted for inclusion in WVU Graduate Theses, Dissertations, and Problem Reports collection by an authorized administrator of The Research Repository @ WVU. For more information, please contact researchrepository@mail.wvu.edu. 


\title{
ASSESSMENT STRATEGIES FOR MIXING IN VERY VISCOUS GAS-LIQUID TWO-PHASE FLOWS
}

\author{
Sharad Chand Ravinuthala \\ Thesis submitted to the \\ Benjamin M. Statler College of Engineering and \\ Mineral Resources \\ at West Virginia University \\ in partial fulfillment of the requirements \\ for the degree of \\ Master of Science \\ in \\ Mechanical Engineering \\ Ismail B. Celik, PhD, Chair \\ John M. Kuhlman, PhD. \\ Wade W. Huebsch, PhD. \\ Department of Mechanical and Aerospace Engineering \\ Morgantown, West Virginia \\ 2014
}

Keywords: Dispersed two-phase flow; Bubbly flow; Mixing; Lagrangian particle tracking; Bubble column experiments; Computational Fluid Dynamics (CFD);

Two-way coupling; Eulerian - Eulerian; Gas-Liquid Flow.

Copyright 2014 Sharad Chand Ravinuthala 


\section{ABSTRACT \\ Assessment strategies for mixing in very viscous gas-liquid two phase flows. \\ Sharad Chand Ravinuthala}

Bottom heating approach for glass melting offers potential benefits of higher efficiency and lower emissions compared to the conventional surface fired melters with burners above the bath surface. Recent advances in the enabling technologies such as burners, controls, heat recovery and refractive materials have led to successful demonstration of bottom heating Submerged Combustion Melting (SCM) of glass. In the reactor, combustion products of natural gas combustion are bubbled through the three phase re-circulating tank reactor. The turbulence generated by the rising bubble column causes rapid heating and mixing of the charge resulting in fast melting and homogeneous composition of the product. Detailed understanding of such two-phase gas liquid flows is imperative for developing efficient multi-phase reactors through precise control of mixing and reaction kinetics. The bubble column, is a good apparatus for an elementary experimental study and numerical modeling of such flows. In this study, the hydrodynamics of the bubble column are investigated to develop strategies for assessment of mixing in the system. For the numerical part two approaches are used:

i) Using a commercial software ANSYS FLUENT with an Eulerian-Eulerian approach to model the bubble and the continuous phase and

ii) Using an in house LES based Navier-Stokes solver with the Eulerian-Lagrangian method which uses the Particle-in-Ball approach for the Lagrangian particle tracking of the discrete phase (bubbles).

The efficacy of these methods in predicting the plume oscillation period (POP) over a wide range of superficial gas velocities is studied. An attempt is made to simulate the effect of viscosity on such flows. An unheated laboratory scale model with a very viscous primary phase is used for experiments, to better understand the effect of viscosity on the hydrodynamics of the bubbles rising and by extension the mixing obtained in the system. 
To My Godfather 'TheMamaiMama', the best and the wisest of men I've ever known.

\&

To Sir Isaac Newton for consistently blowing my mind. 


\section{Acknowledgements}

I wish to express my heartfelt gratitude to the wonderful teachers I have had during the course of my education, the list of whom would always be lead by my father Sri. Ramakoteswara Rao. My success, if any, lies in direct correlation with their kind, nurturing and feeding my curiosity.

I wish to thank Dr.Nithi Sivaneri for welcoming me to Morgantown, WV with a generous TA offer, my advisor Dr.Ismail Celik for his financial and academic support and Dr.Robin Hensel for the financial support during my initial days at WVU.

This work would have never attained completion without the staunch support of my girlfriend Sucharitha Koleti and my brother Shailend Chand, the luxury of their unconditional love I always had. I wish to thank my friends Harsha Chand Kolli, Meghana Ramakumar and others for ensuring I always had a family this far from home.

Then there are people who played a major role in making my academic life at WVU memorable. I would like to extend my heartfelt gratitude to the following friends starting with Dr.Raju Pakalapati who helped me find my feet at the CFD \& AMP centre; Dr. Steve Rowan for his assistance with experiments; Dr. Gusheng $\mathrm{Hu}$ for his timely correspondence on matters concerning the DREAM code, Sergio Escobar for his genuine helping nature that I found infectious; Jose Escobar for his wonderful insights on issues that gave me a hard time and whose level of rigour I always found inspiring; Hayri Sezer whose 'Beautiful Mind' I had the good fortune to experience, with him I could discuss everything from evolution to philology and from quantum computing to computational finance; Gennaro Campitelli, in depth discussions with him about aerodynamics and Formula 1, and the daily technical banter helped me remind myself, during trying times, the primary reason why I had signed up to be a researcher in this field and why I was staying up late. Lastly, I would like to thank my committee members Dr.Wade Huebsch and Dr. John Kuhlman, a special thanks to Dr. Kuhlman for his famous "Introduction to Turbulence" course, attending which I consider one of the high points of my graduate life. 


\section{Contents}

$\begin{array}{lll}1 & \text { Introduction and Background } & 7\end{array}$

1.1 Modelling of Glass Melters . . . . . . . . . . . . . . . . . 7

1.2 CFD modelling: . . . . . . . . . . . . . . . . . . . . . . 12

1.3 Benefits of Bottom Melting . . . . . . . . . . . . 13

1.4 Objective ............................. 14

2 Modeling Strategies for Simulations $\quad 15$

2.1 The Bubble Column . . . . . . . . . . . . . . . . . 15

2.2 Numerical Modelling . . . . . . . . . . . . . . . . . . 17

2.2.1 The Governing Equations for Fluid Flow . . . . . . . . . . 17

2.2.2 Strategies for Turbulent Flows . . . . . . . . . . . . . . . 21

3 Formulation of Multiphase-Flow Models 30

3.1 Hydrodynamic Forces on a rising bubble . . . . . . . . . . . . . 30

3.1.1 The non-dimensional groups . . . . . . . . . . . . . 31

3.1 .2 The Forces . . . . . . . . . . . . . . . . . . . . . . . 33

3.2 Eulerian Multiphase-Flow Models . . . . . . . . . . . . . . 39

3.2.1 The Mixture Model . . . . . . . . . . . . . . . . . . . . . . . 39

3.2.2 Multi Fluid Eulerian Model . . . . . . . . . . . . . . . . 42

3.3 The Eulerian Lagrangian Method . . . . . . . . . . . . . . . . . . 44

4 Results and Discussion $\quad 48$

4.1 Eulerian-Eulerian Simulations . . . . . . . . . . . . . . . . 48

4.2 Eulerian-Lagrangian Simulations _. . . . . . . . . . . . . . 60

4.3 Experiments . . . . . . . . . . . . . . . . 68

4.3 .1 Very Viscous Case . . . . . . . . . . . . . . . 68 
4.3.2 Results and Analysis . . . . . . . . . . . . . . . . 73

5 Conclusions and Future Work $\quad 90$

5.0 .3 Conclusions . . . . . . . . . . . . . . . . . . 90

5.0 .4 Future Work . . . . . . . . . . . . . . . . . . . 92

Appendix A User defined function for the drag law . . . . . . . . . . . . 99

Appendix B Bubble size distribution in $\mathrm{cm}$. . . . . . . . . . . . . . . 102 


\section{List of Figures}

1.1 Schematics for Submerged Combustion Melting; (a) by (Ross \& Tincher, 2004) (b) by David Rue . . . . . . . . . . . . . . . . 10

3.1 Particle near wall . . . . . . . . . . . . . . . . . . . . 37

3.2 The Coanda effect . . . . . . . . . . . . . . . . . . . 37

4.1 (a)Dimensions of the setup used by (Diaz et al. , 2008) (b) the coarse grid (c) the refined mesh . . . . . . . . . . . . . . . 52

4.2 Calculated liquid velocity and the corresponding power spectral density function at different flow rates compared with results of (Diaz et al. , 2008), reproduced with permission. . . . . . . . . . 54

4.2 (Concluded) Calculated liquid velocity and the corresponding power spectral density function at different flow rates compared with results of (Diaz et al. , 2008), reproduced with permission. . . . . . . 55

4.3 Comparison between calculated and experimental plume oscillation period, for E-E simulations and experiments by (Diaz et al. , 2008) 56

4.4 Contours of volume fraction of air in water at different $U_{g}$. (a,d,g) experimental (Diaz et al. , 2008);(b,e,h) computational (Diaz et al. , 2008); (c,f,i) computational present work . . . . . . . . . . 59

4.5 Grid used in the Eulerian-Lagrangian simulations . . . . . . . . . . 60

4.6 Comparison between calculated and experimental plume oscillation period, for E-L simulations and experiments by (Diaz et al. , 2008) 63

4.7 The Uniform Grid used for the simulation of a viscous case. $74 \times 36 \times 1664$

4.8 Calculation of liquid velocity and the corresponding power spectral density . . . . . . . . . . . . . . . . . . . 6 . 65

4.9 Gas Holdup vs Time . . . . . . . . . . . . . . . . . . 67 
4.10 The experimental setup . . . . . . . . . . . . . . . . . . 69

4.11 Digital photographs of gas pockets in the viscous liquid media, The binary images obtained through edge detection and image processing from image $[1 \mathrm{SCFH}] \ldots$. . . . . . . . . . . . . . 74

4.11 (Continued) Digital photographs of gas pockets in the viscous liquid media, The binary images obtained through edge detection and image processing from image $[2-6 \mathrm{SCFH}] \ldots \ldots$. . . . . . 75

4.11 (Continued) Digital photographs of gas pockets in the viscous liquid media, The binary images obtained through edge detection and image processing from image $[8-10 \mathrm{SCFH}] \ldots \ldots . . . .76$

4.11 (Continued) Digital photographs of gas pockets in the viscous liquid media, The binary images obtained through edge detection and image processing from image $\mathrm{J}[11-14 \mathrm{SCFH}] \ldots \ldots . . . . .77$

4.11 (Concluded) Digital photographs of gas pockets in the viscous liquid media, The binary images obtained through edge detection and image processing from image $[16-18 \mathrm{SCFH}] \ldots \ldots . . .78$

4.12 Variation of the Harmonic Mean Diameter . . . . . . . . . . . . . 79

4.13 Variation of small bubble distribution . . . . . . . . . . . . . 79

4.14 Variation of the Sauter Mean Diameter . . . . . . . . . . . . . 81

4.15 Particle size (in pixels) distribution for[2-14 SCFH] from the image analysis using ImageJ. . . . . . . . . . . . . . . . . . 82

4.15 Particle size (in pixels) distribution for [18-20 $\mathrm{SCFH}]$ from the image analysis using ImageJ. . . . . . . . . . . . . . . . . 83

4.16 Analysis of the differential pressure signal and its corresponding power spectral density $[2-8 \mathrm{SCFH}] \ldots \ldots$. . . . . . . . . 85

4.16 (Continued) Analysis of the differential pressure signal and the power spectral density[10-16 SCFH $] \ldots \ldots$. . . . . . . . 86

4.16 (Concluded) Analysis of the differential pressure signal and its corresponding power spectral density $[20 \mathrm{SCFH}] \ldots \ldots$. . . . 87

4.17 Variation of Global Gas Holdup with $U_{G S}(\mathrm{~m} / \mathrm{sec}) \ldots \ldots \ldots . . .87$

4.18 Regime mapping on the chart by (Clift et al. , 1988) based on equivalent diameter (de) for Silicone Fluid and Glycerin. . . . . . . . 88 
1 Particle size (in $\mathrm{cm}$ ) distribution for[1-2 SCFH] from the image analysis using ImageJ. . . . . . . . . . . . . . . . . . . . . . . 102

1 Particle size (in $\mathrm{cm}$ ) distribution for [6-10 SCFH] from the image analysis using ImageJ. . . . . . . . . . . . . . . . . . . . . 103

1 (Concluded)Particle size (in $\mathrm{cm}$ ) distribution for[11-18 $\mathrm{SCFH}]$ from the image analysis using ImageJ. . . . . . . . . . . . . . . . . . 104 


\section{List of Tables}

4.1 Inlet velocity values for a given superficial gas velocity. . . . . . . . 50

4.2 Solutions methods, spatial and temporal discretizations . . . . . . . 51

4.3 Turbulence Model Parameters . . . . . . . . . . . . . . . . . . 51

4.4 Comparision of calculated frequencies with (Diaz et al. , 2008) . . . 53

4.5 Scaled Diameter for E-L Simulation . . . . . . . . . . . . . . . . 62

4.6 Pure Silicone Fluid (Polydimethylsiloxane) Data . . . . . . . . . . . 70

4.7 Conversion of flow rate from SCFH to $U_{G S} \ldots \ldots$. . . . . . . . . . . 80 


\section{Chapter 1}

\section{Introduction and Background}

\subsection{Modelling of Glass Melters}

For decades tank melters have been used to produced most of the industrial glass. In spite of improvements in the concerned technology, such as burners, controls, and heat recovery the core process has by far remained the same. Recently, several efforts by researchers and glass companies have been made to replace the tank melter. They have been well documented in the 2004 report Glass Melting Technology : A technical Assessment (Ross \& Tincher, 2004) commissioned by the U.S. Department of Energy (USDOE), which provides a history of these efforts, what follows is an excerpt from the same. One such approach discussed is, PPG's P-10 process. This glass formation process consists of four distinct processing devices: batch preheater ; glass melter (primary melter) ; glass dissolver (secondary melter) , vacuum refiner. The raw materials here are preheated using the waste heat from the melting phase and de-carbonated in the rotary kilns. The products are then fed into a rotary melting pot that holds them on the walls through the centrifugal force, thus eliminating the need for a refractory lining. A central torch heats and melts the batch off the wall, which flows through the bottom of the pot. The dissolution of the batch happens in a shallow canal heated through electrodes or a series of compartments heated through submerged oxy-hydrogen burners. By decreasing the pressure above the glass to 20-40 millitorr, refining of the glass can be achieved. At this low pressure the trapped gases bubble out easily thus resulting in bubbling and foam. Rapid pressure changes, burners and direct water injection 
are used to break up this layer of foam. The P-10 melting system installed at PPG facilities, showed that the system met the goals for which it was developed. At the time the project was started energy required for a flat glass furnace was about 6 million BTU/ton. The theoretical consumption was 2.2 million. Despite the technological accomplishments, the units experienced operational instability, and procurement of oxygen became more expensive than using natural gas.

In industrial glass melting processes reducing the energy consumption has become the priority. In the case of gas melting through conventional tank melters, the energy transfer is maximum in the batch blanket zone where $75-90 \%$ of the total required heat for melting is taken in by the batch in 45-60 min (Beerkens, 2004). The fining process then follows where the melt is brought to high temperatures to accommodate the removal of dissolved gases. This is followed by a re-fining process which is a controlled cooling process in which the remaining small gas bubbles are reabsorbed and then conditioning where the chemical and thermal homogenization of the melt is ensured. The aforementioned steps, where the mixing and homogenization takes place, primarily govern the residence time requirements for the furnace. The average residence time multiplied by the production rate gives a measure of the volume that sets the furnace size. The average residence times are $24 \mathrm{hrs}$ for container glass and greater than 60 hours for TV-panel glass melts. The average residence time is a good measure of the minimum residence time; which in turn characterizes the glass quality for the melt, with the minimal residence time follows the trajectory in the tank with the lowest temperatures and is thus critical. The essential steps in the conversion of batch to homogeneous glass melt are now discussed in detail (Beerkens, 2004):

\section{- Batch Heating:}

At this stage the batch behaves almost like a solid and is in a plug flow regime. Energy transfer is intense and is required to heat the batch to 1250$1300 \operatorname{deg}$ C. Tank furnaces require a strong return flow of the glass melt, since this facilitates the transfer of energy from the hottest regions of the tanks, so in a way the melt itself acts as a energy carrier. Using heating electrodes does assist, but at the expense of additional energy input. 


\section{- Sand Grain Dissolution:}

The temperature in this stage must be high enough to raise the $\mathrm{SiO}_{2}$ solubility of the melt above the actual $\mathrm{SiO}_{2}$ concentration of the melt, to enable dissolution. But this temperature has to be lower than fining onset temperature ( 1350-1400 deg C). The required minimum dissolution times largely depend on the temperature, the grain size distribution and the glass melt composition.

\section{- Gas Bubble Removal:}

Fining requires a minimum residence time of $2-3 \mathrm{~h}$ in the fining zone. Fining also needs a flow that is almost plug in nature, undisturbed and shallow in depth. Gas bubbles that are not able to make it to the surface in the primary fining should be reabsorbed by the melt since the solubility of a large number of gases decrease with an increase in glass temperature.

\section{- Homogenization}

In the absence of convection and velocity gradients, the difference in concentration of a cord compared with that of the parent glass melt is smoothed out through diffusion alone, which is an inherently slow process. It can then also be said that the process of homogenization needs strong mixing to cause the elongation of the glass inhomogeneties through stretching convection patterns. Low temperatures are required, but not too low, because the viscosity needs to be low even as the molecular diffusion needs to be high. The time required for homogenization can be reduced by a larger order if one were to introduce mechanical mixing, but it needs to be ensured that all the melt passes through close vicinity of the mixers for effective mixing. The residence time in the homogenization (due to stirrers) stage is lesser compared to the average residence time of the glass melt. This form of homogenization is prominent in making of TV, optical and float-glass melts.

A segmented approach was also proposed (R. Beerkens, 2004), known as modular melting. In this approach an attempt was made to separate the above men- 


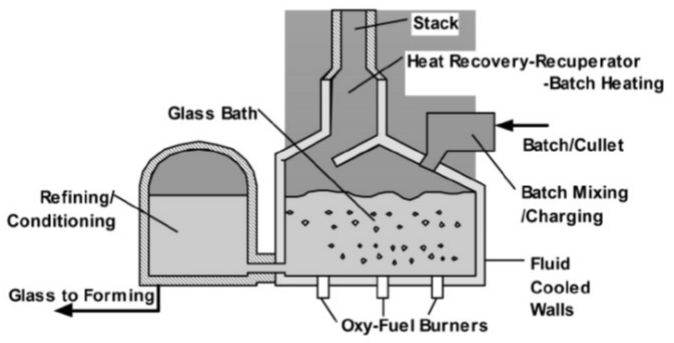

(a)

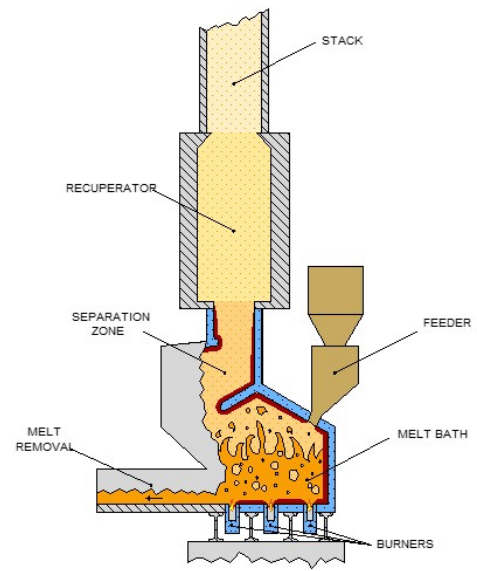

(b)

Figure 1.1: Schematics for Submerged Combustion Melting; (a) by (Ross \& Tincher, 2004) (b) by David Rue

tioned process steps physically by construction of segmented melters. This shortened the total time for melting and refining, but the additional complexity of the process was counterbalanced out by the higher efficiency and greater process flexibility.

Another approach, which is also the prime motivation of the present effort is the bottom heating for glass melting through Submerged Combustion Melting (SCM). This process uses forced convection and direct contact heat transfer to create high intensity heat transfer and rapid homogenization. Recent work by the Glass Technology Institute (GTI) and a set of consortium companies has resulted in moving this concept to the cusp of commercialization.

The Gas Institute of the National Academy of Sciences, Ukariane (GINASU) also undertook the development of SCM, an effort that included the development of highly reliable water-cooled burners and a burner configuration that cause the best possible mixing in the molten pool. The advantage in using these submerged burners results from the fact that they offer rapid heat release, but with an additional advantage of convective mixing and melt homogenization.

The GINASU team set a melt pool depth between 0.7 and $1.5 \mathrm{~m}$ to optimize heat transfer and to promote homogenization (Rue \& Brown, 2011). The melt chamber had a rectangular design 1.1a, with the batch charged at one end and the 
melt discharged at the other. SCM has a higher heat flux through the wall than a conventional melter, but that is compensated for through a smaller size; and overall it is predicted that there will be a drop of $40-50 \%$ of energy consumption on a per pull basis.

In the 1990s GTI planned to augment this technology with advanced controls and moving to oxygen-gas firing from air firing, this becoming viable due to a drop in oxygen costs world wide. The benefits of this shift seem to be multi fold; a decrease in exhaust gas volume of up to $90 \%$, eliminating nitrogen increased heat transfer rate to the melt, NOx emissions would also be lower. The melt bed agitation, however, drops greatly with oxygen firing, while still retaining enough driving force for the required high intensity mixing and melt homogenization. The convective mixing offered by these burners into the melt serve many important purposes; the mixing establishes residence time patterns in the melt and the mixing of the hot glass with cooler glass is the most important form of energy transfer in the process which results in a uniformly heated glass. However, an initial effort to sell this technology to the mineral wool industry in the US proved futile.

This technology was rejected by the industry on three counts (Rue \& Brown, 2011);

- i) issues related to the newly added cost of oxygen

- ii) Reliability of the melter (there was no history of such a process in the country)

- iii) higher capital costs involved with this technology. As all the mineral wool industries used coke-fired cupolas.

In the late 1990s, the USDOE along with glass manufacturers formed a consortium for the development of the bottom melter for transformational Next Generation Melting System (NGMS)(Ross \& Tincher, 2004)1.1b. This consortium later, actively pursued the fundamental understanding of the bottom melting concept through cold flow models, hot melt tests and the development of a 3 phase FLUENT computational fluids dynamics (CFD) model. Cold flow tests were conducted with glycerin and dye in Plexiglass models with air jets, which showed the burner pattern and burner spacing both played an important role in obtaining optimal 
convective mixing. CFD modeling went a little further to determine the optimal melter shape, temperature profiles, refractory thickness to minimize heat loss, feeder location and the position of the exhaust duct. The work of this consortium ended with the demonstration of an energy-efficient bottom metler that produced homogenous glass that contained 25-30\% void fraction of bubbles which ranged from $1 \mu-\mathrm{m}$ to several millimeters. This glass would then need refining through an appropriate refining process.

\subsection{CFD modelling:}

The variables that govern these melting processes are varied in number. It is possible to obtain a homogenous melt with very little un-melted particles, with very little volatilization and enhanced thermal efficiency if the physics is thoroughly understood and modeled. The design iterations that might be needed to arrive at the optimal parameters would include ; different chamber shapes, varying melt depth, the number of burners, the pattern of the burnerns, the design of the burners, feed, exhaust gas, melt discharge locations etc.

Since physically testing the effects these parameters would be prohibitively difficult and expensive, the aid of CFD is sought. A three phase Fluent CFD code, was developed jointly by the FLUENT, Inc. and the consortium companies (Rue \& Brown, 2011). The code must successfully handle a complex three phase flow, resolve the time scales associated with fast gas plumes, the chemistry associated with the process, and some relatively slower process like the convective mixing and the frozen layer interactions near the wall. The code must also be able to deal phenomena thats unique with the bottom melters like the movement of bubbles, the range of sizes of the bubbles, the radiation from the participating media, etc. The modelers have dealt with these through a three step approach:

Step 1: The fast combustion reactions are solved first with estimated initial conditions for a single burner. The model then carries out time averaging of volume of fluid (VOF) solutions over multiple burners; defines gas bubble regions in the bath; defines gas regions above the bath; and defines the local momentum and energy source terms.

Step 2: The inputs from step 1 are used to resolve the glass chemistry, 
bubble behaviour and bath flow. Results from this step are then used to improve the estimates for step 1. This loop is repeated until a stable solution is attained.

Step 3: The stable solution from step 1 and step 2 are used for step 3 where the bulk melter modeling is completed. It is the bulk modeling that helps determine the accounts for melter shape, particle tracking and residence times etc.

\subsection{Benefits of Bottom Melting}

SCM, by the virtue of firing directly into the melt lowers the peak flame temperatures, and has been shown to produce NOx emissions to as low as .05-.1kg/t (Rue \& Brown, 2011) while melting borosilicate glass. Bottom melting can also eliminate the use of fining agents that add cost and contribute to emissions. Bottom melting creates enough turbulence to remove the finest and the hardest of the seeds to remove, the residual from silica grains going into solution and this contributes to more rapid refining, and hence lower energy utilization. The enhanced mixing due to turbulence also accelerates the melting and homogenization process.

In traditional furnaces generally a two roll pattern of slow, convective mixing shows up. In such cases the melting process is hastened by increasing the convective mixing by placement of additional electric heating elements beneath the melter or through bubbling to stir the melt. In the case of SCM though, the massive turbulent mixing breaks up the boundary layers around the unmelted particles thus enabling their faster heating and melting. It was also observed that the SCM offsets the peak temperatures by almost $100 \mathrm{deg} \mathrm{C}$ when compared to the conventional melters.

As expected then the benefits of high intensity mixing are unsurprising, as SCM with either Oxygen or Air firing produces a homogeneous melt with a residence time of 2-4 hours. The level of mixing obtained with Oxygen is similar what is obtained with Air in spite of $80 \%$ reduction in exhaust gases. The violent mixing induced by submerged combustion then has resulted in a level of mixing never before seen in glass melting. Analysis of glasses made thus, have shown absence of cords, streaks, stones, cord sacks or remnants of silica batch. 


\subsection{Objective}

The objective and motivation of the present effort is to gain a detailed understanding of two-phase gas liquid flows, which is imperative for numerical modelling and developing efficient multi-phase reactors through precise control of mixing and reaction kinetics.

In the current study, the flat, centrally aerated bubble column is taken as a good starting point for elementary studies of such flows. The flat (rectangular) bubble column, retains the key characteristics of a cylindrical column without the accompanying computational overhead associated with modeling and discretizing a cylindrical bubble column. Two computational models based on the EulerianEulerian and the Eulerian-Lagrangian approaches are used for the simulation of unheated transient two phase air in water flows in a bubble column, and the results were validated using the experimental and computational data already published in literature.

Experiments are also conducted to assess the effect of liquid viscosity on flow dynamics inside a bubble column. Silicone fluid is used as the continuous media, and air being the dispersed phase. The objective of this effort are to use the results for a qualitative validation of the numerical simulations, and to prescribe a methodology for successful prediction. The long term goal is to further refine the CFD model for the simulation of three-phase submerged combustion and melting, such as that used in the glass-industry discussed above. 


\section{Chapter 2}

\section{Modeling Strategies for Simulations}

\subsection{The Bubble Column}

A bubble column is a an apparatus containing liquid; with a comparatively smaller dimension depthwise, and of varying types of cross sections. For the sake of our numerical simulations we consider a rectangular cross section as used by (Diaz et al. , 2008). A typical bubble column consists of gas spargers mounted on the bottom of the setup, through which the gas bubbles enter the column, and sets the initially quiescent liquid into motion. Since the bubbles that enter the setup are due to a continuous supply of air, they constitute a plume. This plume or a bubble hose (Becker et al. , 1999) tends to slowly move in the lateral direction. The immense circulating flow of the liquid phase tends to change the flow direction due to the movement of the bubble hose, which results in the plume meandering in a periodic fashion. The prediction of this dynamic behaviour is important to characterize mixing.

Based on the type of gas injection, the bubble columns could be categorized as either partially aerated or fully aerated. The current case is that of a partially aerated column, with the aeration located at the center of the column henceforth termed as centrally aerated or symmetrically aerated. Due to the simplicity of construction and study the bubble column has been a perennial favourite of the scientific community, especially the multiphase CFD community. As a result, in 
due course of literature, the bubble column has been widely studied with no dearth of reliable experimental data. It has also been often used as a standard test to validate the multiphase flow models and numerical solutions. The setup albeit simple, incorporates complexities that manifest in the wide variety of the hydrodynamic forces acting on the bubble [Sec.3.1.2] like gravity, drag, lift, buoyancy, fluid stresses, added mass forces; neglecting any of which would cause an erroneous behaviour of the numerical model.

The bubble column presents itself as a classical case of a two-way coupling model. A two-way coupling problem is when both, the effects of particles on the flow and the effect of the flow on the particles, are accounted for. And finally, this setup provides a crucial benchmark in evaluating the efficacy of the EulerianEulerian and the Eulerian-Lagrangian models in dealing with such problems. Both of the approaches shall be discussed at length in the following sections. ( $\mathrm{Hu}, 2005)$ raises and answers three important questions concerning fluid-dynamic modeling of these two phase flows.

(i) Which approach is more suitable? The E-E (Eulerian-Eulerian) or E-L (EulerianLagragian)

(ii) Which governing equations should be used ?

(iii) Must a turbulence model be included ? or would a laminar treatment suffice ?

The answers to the above questions were as follows:

i) It was noted by $(\mathrm{Hu}, 2005)$ that the majority of the simulation works in literature had taken E-E path. Few that adopted the E-L approach were [(Delnoij et al. , 1997a,b)]. In theory, if both these models accounted for similar terms with the numerical discretization being sufficiently fine, then the results must be comparable. Such a presumption then brings to light the numerical diffusion that is inherited in the case of an E-E approach; which would make the dispersion of the particle (bubble) scheme dependent. Such a scenario is completely avoided in the case of an E-L approach, but not without a trade off, and that being in the for of the computational expense that caps the number of particles that can be tracked at a given time.

ii) The governing equations for the liquid phase are decided upon by the approach chosen and the formulation of the model. For example, with the E-E approach 
a volume averaged equation or a mixed fluid equation are often seen. With the E-L approach the volume averaged equation or the Reynolds-Averaged equation is often used for the primary fluid, along with the equation of the motion for the discrete phase (bubbles) is used.

iii) In the occasion of turbulence being considered, the most widely used turbulence models are of the RANS kind, the $\kappa-\epsilon$ model finds wide applicability in these flows, the veracity and drawbacks of which, in these scenarios, has been discussed in detail by (Sokolichin \& Eigenberger, 1999). Such models formulate an eddy viscosity termed as $\mu_{t}$ where

$$
\begin{gathered}
\mu_{t}=C_{\mu} \rho_{l} \frac{\kappa^{2}}{\epsilon} \\
\mu_{e f f}=\mu_{t}+\mu_{l}
\end{gathered}
$$

It also must be noted that the $\kappa-\epsilon$ model usually induces excessive damping that results in overprediction of viscosity; such situations are negotiated with a prescription of empirical viscosities. The effects of turbulence can also be altogether ignored by an "audacious" step of neglecting the effects of turbulence and thus implicitly stating that the effective viscosity is equal to the viscosity of the fluid itself.

$$
\mu_{\text {eff }}=\mu_{l}
$$

Such an assumption though, is seldom justified. And as we shall further see, all the physics prevalent in these bubble columns are predominently turbulent in nature. Thus a 'laminar' assumption might, in most cases, lack a sound theoretical justification.

In the present effort the E-L and the E-E approaches are examined in their ability to handle the above problem. The approaches are discussed in further detail in Section 3.2.

\subsection{Numerical Modelling}

\subsubsection{The Governing Equations for Fluid Flow}

A true natural phenomena has different layers/levels of complexities and physics pertaining to each such layer. Hence a detailed representation or understanding 
of such phenomena ends up being an educated task of choosing the level of depth that must be represented and is of interest. For example, various descriptions of our physical world have been proposed from subatomic, atomic to microscopic, macroscopic and to astronomic. Depending on the nature of the problem and level of interest a mathematical "model" can be derived, with certain assumptions, and said to be representative of the true physics of it all. It is then of real interest to note the usage of the term "model" instead of "solution", the motivation for which shall become apparent with further discussion.

The laws of classical fluid dynamics have been well established, the underlying premise of which is the continuum hypothesis; which assumes that the fluid elements considered are much larger than the mean free path of the fluid molecules. They further state that during the motion of a fluid, certain properties such as mass, energy and momentum are conserved or in other words neither created nor destroyed. This then gives us a set of differential-equations (mathematical model) to work with in an effort to define and solve the dynamics of flow (the problem under consideration). These equations under special considerations constitute the well known and oft celebrated Navier-Stokes (N-S) equations, which have made themselves paramount in a solution to many fluid flow problems. What follows is a brief on the origin of these equations; for more details the reader is directed to classical texts such as (Schlichting \& Gersten, 2000) and (White, 2006).

Let $\phi$ be a generic intensive property that stands conserved in a control mass. A few examples of $\phi$ are density (mass per unit volume), velocity (momentum per unit mass) and scalars like energy, pressure and temperature. Now if we consider a corresponding extensive property $\Phi$, whose value varies in relation to the mass can be expressed as

$$
\Phi=\int_{\Omega_{C M}} \rho \phi d \Omega
$$

Where $\Omega_{C M}$ is the volume occupied by the control mass, $\rho$ is the density. If $\mathbf{V}$ is the flow velocity and $\mathbf{V}_{\mathbf{C S}}$ is the velocity with the the control surface CS moves; then the integral differential equation that describes the flow is given by

$$
\frac{d \Phi}{d t}=\frac{d}{d t} \int_{C V} \rho \phi d \Omega+\int_{C S} \rho \phi\left(\mathbf{V}-\mathbf{V}_{\mathbf{C S}}\right) \cdot \mathbf{n} d S
$$


Where $\mathbf{n}$ is the unit normal to the CS and directed outwards. A special case then arises for a stationary control volume $\left(\mathbf{V}_{C S}=0\right)$. By the virtue of the the fact that $\mathbf{V}=f(\mathbf{r}, t)$ and $\mathbf{V}_{C S}=f(t)$, the first integral on the right hand side then becomes a local time derivative from a total time derivative. Hence

$$
\frac{d \Phi}{d t}=\frac{\partial}{\partial t} \int_{C V} \rho \phi d \Omega+\int_{C S} \rho \phi(\mathbf{V}) \cdot \mathbf{n} d S
$$

If $\phi$ is taken to be unity the equation takes the form of mass conservation (continuity); if $\phi=\mathbf{V}$ then the equation takes the form of momentum conservation; if $\phi=$ energy then it becomes the energy conservation equation.

$$
\begin{gathered}
\frac{\partial}{\partial t} \int_{C V} \rho d \Omega+\int_{C S} \rho(\mathbf{V}) \cdot \mathbf{n} d S=0 \\
\frac{\partial}{\partial t} \int_{C V} \rho(\mathbf{V}) d \Omega+\int_{C S} \rho(\mathbf{V})(\mathbf{V}) \cdot \mathbf{n} d S=\sum_{l} \mathbf{f}_{l} \\
\frac{\partial}{\partial t} \int_{C V} \rho(e) d \Omega+\int_{C S} \rho(e)(\mathbf{V}) \cdot \mathbf{n} d S=S_{\phi}
\end{gathered}
$$

In the above equations the right hand side term in Eq. 2.8 represents all forces (surface forces and body forces) acting on the control volume CV; $S_{\phi}$ represents all these sources and sink terms that contribute to energy transfer rates. With the aid of the Gauss' divergence theorem the surface integral is transformed into a volume integral and the result leads to the Navier-Stokes equations:

$$
\begin{gathered}
\frac{\partial \rho}{\partial t}+\operatorname{div}(\rho \mathbf{V})=0 \\
\frac{\rho \mathbf{V}}{\partial t}+\operatorname{div}(\rho \mathbf{V} \mathbf{V})=\operatorname{div}(\sigma)+\rho \mathbf{b} \\
\frac{\rho h}{\partial t}+\operatorname{div}(\rho h \mathbf{V})=\operatorname{div}(k \nabla T)+\frac{D p}{D t}+\psi+S_{\phi}
\end{gathered}
$$

Where $\sigma$ is the stress tensor due to the surface forces, and $\mathbf{b}$ is the body force per unit volume, $\mathrm{T}$ is the temperature, $\mathrm{h}$ is the enthalpy, $\mathrm{p}$ is the pressure, $\psi$ is the viscous dissipation function which manifests in the dissipation of the mechanical energy into thermal enegy. The first term on the left of the above equations represents the local transient nature of the system, the second term on the left shows the convective or the advective effects in the system. The convective term could in other words be termed as transport due to velocity, and as we see in the case of the 
continuity equation it is transport of mass by velocity, in the momentum equation it is transport of momentum by velocity and finally in the energy equation it is the transport of energy by velocity. The first term on the right hand side represents the effect of diffusion viz. viscous and thermal effects respectively.

The Cartesian coordinate system shall be the one used henceforth here, and using the standard tensor notation the Navier-Stokes (continuity and momentum) equations take the form:

$$
\begin{gathered}
\frac{\partial \rho}{\partial t}+\frac{\partial}{\partial x_{i}}\left(\rho V_{i}\right)=0 \\
\frac{\left(\rho V_{i}\right)}{\partial t}+\frac{\partial}{\partial x_{j}}\left(\rho V_{j} V_{i}\right)=-\frac{\partial p}{\partial x_{i}}+\frac{\partial \tau_{i j}}{\partial x_{j}}+\rho b_{i} \\
\frac{\rho h}{\partial t}+\operatorname{div}(\rho h \mathbf{V})=\operatorname{div}(k \nabla T)+\frac{D p}{D t}+\psi+S_{\phi}
\end{gathered}
$$

where

$$
\begin{gathered}
\tau_{i j}=2 \mu S_{i j}-\left(\lambda \frac{\partial u_{j}}{\partial x_{j}}\right) \delta_{i j}, \lambda=\frac{2}{3} \mu \\
S_{i j}=\frac{1}{2}\left[\frac{\partial u_{i}}{\partial x j}+\frac{\partial u_{j}}{\partial x_{j}}\right]
\end{gathered}
$$

Here $\tau_{i j}$ is the viscous stress tensor, $S_{i j}$ is the strain rate tensor. The indices i and $\mathrm{j}$ represent the main and dummy index respectively; and take values from 1 through 3 representing the three spacial directions.

In the above representations, the energy equation has been left out as the current study concerns itself with isothermal flows.

It should be stressed that, in the event that the flow is incompressible in nature then the continuity equation simplifies to:

$$
\frac{\partial}{\partial x_{i}} V_{i}=0
$$

which means that the shear stress term $\frac{\partial \tau_{i j}}{\partial x_{j}}$ could be written as follows

$$
\frac{\partial \tau_{i j}}{\partial x_{j}}=\frac{\partial}{\partial x_{j}}\left(2 \mu S_{i j}\right)=\frac{\partial}{\partial x_{i}}\left(2 \mu \frac{1}{2}\left[\frac{\partial V_{i}}{\partial x j}+\frac{\partial V_{j}}{\partial x_{j}}\right]\right)
$$

invoking continuity Eq.2.18

$$
\frac{\partial}{\partial x_{j}}\left(\mu \frac{\partial V_{j}}{\partial x_{i}}\right)=\mu \frac{\partial}{\partial x_{i}}\left(\mu \frac{\partial V_{j}}{\partial x_{j}}\right)=0
$$


That gives us the final form of the momentum equation that we shall largely concern ourselves with in due course of this study

$$
\frac{\left(\rho V_{i}\right)}{\partial t}+\frac{\partial}{\partial x_{j}}\left(\rho V_{j} V_{i}\right)=-\frac{\partial p}{\partial x_{i}}+\frac{\partial}{\partial x_{j}}\left(\mu \frac{\partial V_{i}}{\partial x_{j}}\right)+\rho b_{i}
$$

\subsubsection{Strategies for Turbulent Flows}

It is worth noting that the aforementioned equations apply to all forms of flows, but due to computational constraints one would need special approaches if turbulence has to be dealt with. Therefore, inorder to deal with or accommodate turbulent flows some special formulations are needed; an overview of these is presented in this section.

\section{Characteristics of turbulence}

Since an all encompassing definition for turbulence is difficult to find, some characteristics of turbulence are discussed below.

1. Turbulence is/ appears to be random and chaotic i.e the system does not repeat itself in time, except for averages. What that essentially means is that the RMS (root mean square) values of fluctuations in velocity/pressure/temperature fluctuations could be repeatable in time!

2. Could be said to be consisting of 'eddies' of various length scales (sizes) and time scales (periods of existence) . The length scales (L) could be ranging from $L_{\max }=f\left(\right.$ geometryof flow) to $L_{\min }=f(\nu, R e)$

3. Is a continuous phenomena, which implies that it is still governed by the famed Navier-Stokes Equations, implying that $L_{\min }>>\lambda($ Meanfreepath $)$.

4. Is said to contain small scale, random vorticity.

5. Is a $3-\mathrm{D}$ phenomena. For, if it was $2-\mathrm{D}$, then vortex stretching couldn't be accommodated for.

6. Occurs at Reynolds Number much larger than one: $R e>>1$ 
7. Always dissipates energy at the smallest eddy scales.

8. The large scales $L_{\max }$ become independent of Reynolds Number, for $R e>>$ 1.

9. The small scales $L_{\text {min }}$ tend towards isotropy, for $R e>>1$.

10. Tends to be intermittent, for $R e>>1$.

11. Is a non-linear phenomena, due to the presence of $U_{j} \frac{\partial U_{i}}{\partial X_{j}}$ term; hence the similarity drawn to chaos.

It could then be surmised that a flow that is turbulent is highly unsteady, 3-D, with high vorticity and enhanced mixing. There is also a notable dissipation of kinetic energy to heat due to the presence of viscosity. As discussed earlier turbulence is also characterized by a range of scales, large to small (Kolmogrov). These small scales are of considerable interest and are determined by kinematic viscosity, of the fluid under consideration and the dissipation rate $\epsilon$

$$
\begin{gathered}
\eta \sim-\left(\frac{\nu^{3}}{\epsilon}\right)^{(1 / 4)} \\
u_{\eta} \sim(\epsilon \nu)^{(1 / 4)} \\
\tau_{\eta} \sim\left(\frac{\nu}{\epsilon}\right)^{(1 / 2)}
\end{gathered}
$$

where $\eta, u_{\eta}$ and $\tau_{\eta}$ denote the Kolmogrov length, velocity and time scales. The Kolmogrov length scale is defined as the smallest eddy capable of independent existence, just before it dissipates due to viscosity. An inviscid estimate relates the dissipation rate, $\epsilon$ to the largest eddies and their fluctuations, $l_{0}$ (also known as the integral scale) and $u_{0}$ respectively.$l_{0}$ is comparable to $l$ and hence to the flow geometry wheras $u^{\prime}$ is comparable to the RMS value of the velocity fluctuations. The estimate for $\epsilon$ is given by:

$$
\epsilon \sim \frac{u^{\prime 3}}{L_{\max }}
$$

With the help of these estimates one can be lead to the appreciation of the need to avoid or promote the accommodation of turbulence in engineering applications. Not surprisingly the, extensive research has been done towards developing numerical methods for turbulence; all of which could be classified broadly into three categories as below 
1. The Reynolds Averged Navier-Stokes (RANS) Equation Model

2. Large Eddy Simulation (LES)

3. Direct Numerical Simulation (DNS)

The Reynolds Averaged Navier-Stokes (RANS) Model This approach primarily deals with the mean flow and the effects of turbulence on it,it begins with the concept of Reynolds Decomposition in which the property is decomposed as the sum of its time averaged (or ensemble averaged) value and the instantaneous fluctuation it carries:

$$
\mathbf{V}=\overline{\mathbf{V}}+\mathbf{v}^{\prime}
$$

Where $\bar{V}$ is the time averaged entity and $v^{\prime}$ the fluctuation. A similar decomposition could also be applied to a generic scalar quanity $\phi$. The thus decompsed property is now substitued into the required equation and the entire equation is time averaged; such a formulation will enable one to see the effect of the fluctuation on the mean flow. Since the mean flow already obeys the continuity equation; the continuity now takes the form:

$$
\begin{aligned}
& \frac{\partial \bar{V}_{i}}{\partial x_{i}}=0 \\
& \frac{\partial v_{i}^{\prime}}{\partial x_{i}}=0
\end{aligned}
$$

Applying the same and time averaging the momentum equation one would have:

$$
\frac{\partial \bar{V}_{i}}{\partial t}+V_{j} \frac{\partial \bar{V}_{i}}{\partial x_{j}}=\frac{1}{\rho} \frac{\partial}{\partial x_{j}}[-\bar{P} \delta_{i j}+\mu\left(\frac{\partial \bar{V}_{i}}{\partial x_{j}}+\frac{\partial \bar{V}_{j}}{\partial x_{i}}\right)-\underbrace{\rho \overline{v_{i}^{\prime} v_{j}^{\prime}}}_{\text {Reynolds stress tensor }}]
$$

The above equation set is termed as the famed 'Reynolds Averaged Navier Stokes Equations' or in short 'RANS'. It is now seen that a new term now arises due to Reynolds decomposition, this could be seen as turbulence exerting a stress on the mean flow! These consist of normal stresses : $\tau_{x x}=-\rho u^{\prime 2}, \tau_{y y}=-\rho v^{\prime 2}$ and $\tau_{z z}=$ $-\rho w^{\prime 2} \&$ shear stresses $\tau_{x y}=-\rho u^{\prime} v^{\prime}, \tau_{y z}=-\rho v^{\prime} w^{\prime}$ and $\tau_{z x}=-\rho w^{\prime} u^{\prime}$. Together they are termed as the Reynolds Stresses and are said to form the Reynolds Stress Tensor. However, a more correct and a physical interpretation is as follows;

$$
(\text { Reynolds Stress })_{i j}=-\rho \overline{v_{i}^{\prime} v_{j}^{\prime}}=-\underbrace{\overline{v_{i}^{\prime}}}_{\mathrm{i}} \underbrace{\left(\rho V_{j}\right)}_{\mathrm{ii}}
$$


Where the term $(i)$ represents the fluctuating momentum per unit mass in ' $\mathrm{i}$ ' direction and the $(i i)$ represents the fluctuating mass flow rate per unit area in ' $\mathrm{j}$ ' direction. Together then, they could said to be standing for instantaneous rate of flow in j-direction of fluctuating i-momentum per unit area, better known as turbulent transport,! or convective momentum transfer due to turbulent eddies. This appearance of these "new" terms is of some concern at this stage, for in order to be able to solve the equations numerically the system of equations must be closed; and that is not the case here. It is then that a need is felt to close these set of equations with an additional set of transport equations to accommodate the newborn turbulent terms. This effort is termed as turbulence modeling, and is generally done through some turbulence models, the most well known one being the $\kappa-\epsilon$ model.

The $\kappa-\epsilon$ model: This particular model focuses on the changes in the turbulent kinetic. The instantaneous kinetic energy could be defined as the sum of the mean and the fluctuating kinetic energies.

$$
K=\bar{K}+k^{\prime}
$$

Where

$$
\begin{aligned}
\bar{K} & =\frac{1}{2}\left(\bar{U}^{2}+\bar{V}^{2}+\bar{W}^{2}\right) \\
k^{\prime} & =\frac{1}{2}\left(u^{\prime 2}+v^{\prime 2}+w^{\prime 2}\right)
\end{aligned}
$$

The governing equation for mean flow energetics is obtained by multiplying $\mathrm{x}$ Reynolds Averaged momentum equation with $\mathrm{U}$, the y component with $\mathrm{V}$ and the z component with W. Summing those up and with the necessary algebraic rigor alongside the use of the divergence theorem and incompressiblility, the integrated mean flow energy equation about a closed volume can be shown as follows. (Mathieu \& Scott, 2000)

$$
\underbrace{\frac{d}{d t} \int \frac{1}{2} \bar{V}_{i} \bar{V}_{i} d v}_{\mathrm{i}}=\underbrace{\int \overline{v^{\prime} i v^{\prime} j} \frac{\partial \overline{V_{i}}}{\partial x_{j}} d v}_{\mathrm{ii}}-\underbrace{\frac{1}{2} \nu \int\left(\frac{\partial \overline{V_{i}}}{\partial x_{j}}+\frac{\partial \overline{V_{j}}}{\partial x_{i}}\right)\left(\frac{\partial \overline{V_{i}}}{\partial x_{j}}+\frac{\partial \overline{V_{j}}}{\partial x_{i}}\right) d v}_{\text {iii }}
$$

Here, the term $i$ is the rate of change of mean flow kinetic energy or $\mathrm{K}$ as denoted earlier. And the term iii represents the viscous dissipation of the mean. It is 
interesting to note is the absence of the effect of turbulence on the this term. It then essentially means that this viscous dissipation term becomes negligible as we move away from the walls. Finally, the $i$ term represents the rate of work done by the Reynolds stresses due to the fluctuations on the mean flow, and is said to be a sink of energy from the mean flow, or the energy taken away from the mean flow, which is in turn supplied to the production of the turbulent kinetic energy.

It then becomes instructive to see the energetics of the total flow. The following equation is obtained by multiplying the Navier-Stokes equation with $\mathbf{V}_{i}$, then performing the Reynolds decomposition and the time averaging one has

$$
\begin{aligned}
& \frac{d}{d t} \int \frac{1}{2} \overline{V_{i} V_{i}} d v=-\frac{1}{2} \nu \int \underbrace{\left(\frac{\partial \overline{V_{i}}}{\partial x_{j}}+\frac{\partial \overline{V_{j}}}{\partial x_{i}}\right)\left(\frac{\partial \overline{V_{i}}}{\partial x_{j}}+\frac{\partial \overline{V_{j}}}{\partial x_{i}}\right)}_{\mathrm{i}} d v+ \\
& -\frac{1}{2} \nu \int \underbrace{\left(\frac{\partial v_{i}^{\prime}}{\partial x_{j}}+\frac{\partial v_{j}^{\prime}}{\partial x_{i}}\right)\left(\frac{\partial v_{i}^{\prime}}{\partial x_{j}}+\frac{\partial v_{j}^{\prime}}{\partial x_{i}}\right)}_{\mathrm{ii}} d v
\end{aligned}
$$

Thus it is seen that, the total mean energy of the flow is dissipated in the form of the term $(i)$ that represents the mean flow viscous dissipation, and the term (ii) that represents the turbulent viscous dissipation. In general, at high Reynolds number the turbulent gradients are high, the turbulent dissipation overpowers the mean flow dissipation component. But, in the near wall region the mean dissipation gains prominence.

The term (ii) then is $\bar{\epsilon}$ or the time averaged $\epsilon$ that occurs in the $\kappa-\epsilon$ turbulence model.

$$
\bar{\epsilon}=\frac{1}{2} \nu \underbrace{\overline{\left(\frac{\partial v_{i}^{\prime}}{\partial x_{j}}+\frac{\partial v_{j}^{\prime}}{\partial x_{i}}\right)\left(\frac{\partial v_{i}^{\prime}}{\partial x_{j}}+\frac{\partial v_{j}^{\prime}}{\partial x_{i}}\right)}}_{\text {ii }}
$$

In a similar manner the equation for the turbulent kinetic energy (TKE) could be derived as follows:

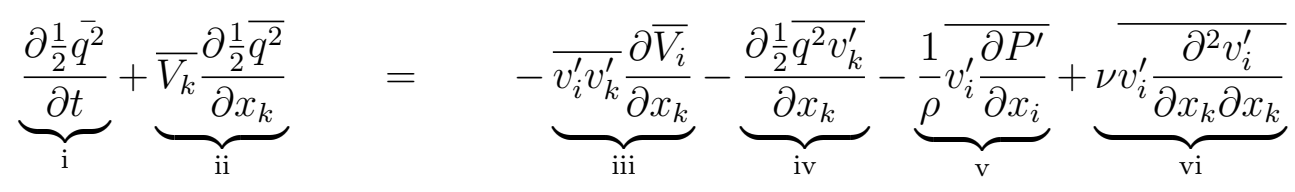


The last term (vi) could be rewritten as thus :

$$
\nu \overline{v_{i}^{\prime} \frac{\partial^{2} v_{i}^{\prime}}{\partial x_{k} \partial x_{k}}}=\underbrace{\nu \frac{\partial}{\partial x_{j}} \overline{v_{i}^{\prime}\left(\frac{\partial v_{i}^{\prime}}{\partial x_{j}}+\frac{\partial v_{j}^{\prime}}{\partial x_{i}}\right)}}_{\operatorname{vi}(a)}-\underbrace{\bar{\epsilon}}_{\text {vi }(b)}
$$

An, essential and elaborate interpretation of the terms is as follows, where $1 / 2 q^{2}$ stands for the TKE $\kappa$ in the $\kappa-\epsilon$ model:

(i) : Represents the local unsteady rate of change of TKE per unit mass.

(ii) : The advection / convection of the TKE by the virtue of the mean flow.

(iii) : Production of TKE by interaction between the mean flow and the turbulence. It is to be noted that $\overline{v_{i}^{\prime} v_{k}^{\prime}}<0$ for cases where there is a positive gradient of the mean velocity; $\frac{\partial \overline{V_{i}}}{\partial x_{k}}>0$. Hence, the negative of that value always turns out to be $>0$; thus the term "production".

(iv) : Transport (advection) of the TKE by the fluctuations, also be looked at as turbulent transport.

(v) : Transfer TKE due to the effects of fluctuation in pressure.

(vi(a)): Viscous transfer of TKE.

(vi(b)): Dissipation of TKE.

A similar transport equation can be conceived for the turbulent dissipation term $\epsilon$ which has the units $m^{2} / s$.

Standard k-e model: The Standard k- $\epsilon$ model (Launder \& Spalding, 1972) has two sets of transport equations, one for the TKE, $\mathrm{k}$, and the other for the dissipation rate $\epsilon$. But, a velocity scale and a length scale representative of the large-scales of the turbulent flow must be determined apriori through dimensional analysis, and are as follows:

$$
\begin{gathered}
\theta=k^{\frac{1}{2}} \\
l=\frac{k^{3 / 2}}{\epsilon}
\end{gathered}
$$

Boussinesq proposed that the Reynolds Stresses might be proportional to the mean rates of deformations:

$$
-\rho \overline{v_{i}^{\prime} v_{j}^{\prime}}=\mu_{t}\left(\frac{\partial V_{i}}{\partial x_{j}}+\frac{\partial V_{j}}{\partial x_{i}}\right)-\frac{2}{3} \rho k \delta_{i j}
$$


where delta $_{i j}$ is the Kronecker delta $(=1$ when $i=j ;=0$ when $i \neq j$ ).

The eddy viscosity for the $\mathrm{k}-\epsilon$ model due to the effects of turbulence is given by :

$$
\mu_{t}=C \rho \theta l=\rho C_{\mu} \frac{k^{2}}{\epsilon}
$$

The following are the transport equations for $\mathrm{k}$ and $\epsilon$ respectively:

$$
\begin{gathered}
\frac{\delta(\rho k)}{\partial t}+\frac{\partial\left(\rho k V_{i}\right)}{\partial x_{i}}=\frac{\partial}{\partial x_{j}}\left[\frac{\mu_{t}}{\sigma_{k}} \frac{\partial k}{\partial x_{j}}\right]+2 \mu_{t} E_{i j} E_{i j}-\rho \epsilon \\
\frac{\partial(\rho \epsilon)}{\partial t}+\frac{\partial\left(\rho \epsilon V_{i}\right)}{\partial x_{i}}=\frac{\partial}{\partial x_{j}}\left[\frac{\mu_{t}}{\sigma_{\epsilon}} \frac{\partial \epsilon}{\partial x_{j}}\right]+C_{1 \epsilon} \frac{\epsilon}{k} 2 \mu_{t} E_{i j} E_{i j}-C_{2 \epsilon} \rho \frac{\epsilon^{2}}{k}
\end{gathered}
$$

where $E_{i j}$ is the mean rate of deformation tensor given by

$$
E_{i j}=\left[\begin{array}{lll}
E_{x x} & E_{x y} & E_{x z} \\
E_{y x} & E_{y y} & E_{y z} \\
E_{z x} & E_{z y} & E_{z z}
\end{array}\right]
$$

The various transport terms which have now begun getting analogous must be interpreted as follows:

(i) : Rate of change of TKE or $\epsilon$.

(ii) : Transport of TKE or $\epsilon$ due to advection

(iii) : Transport of TKE or $\epsilon$ through diffusion

(iv) : Rate of production of TKE or $\epsilon$

(v) : Rate of distruction of TKE or $\epsilon$

Where the constants assume the following values:

$C_{\mu}=0.09$

$\sigma_{k}=1.00$

$\sigma_{\epsilon}=1.30$

$C_{1 \epsilon}=1.44$

$C_{2 \epsilon}=1.92$

Large Eddy Simulation The primary drawback of the Reynolds Averaged Navier Stokes (RANS) equation approach, which has been discussed in the aforementioned section, remains the uniform treatment of all forms of fluctuations. It must be kept in mind that turbulence in its most general form occurs over a wider 
range of length scale of eddies. The small scales of turbulence tend to be isotropic in nature (exhibit similar behaviour independent of direction) and have a nearly universal behaviour. However, on the large scales which are primarily responsible for the energy to be drawn from the mean flow and transfer to the smaller scales, are NOT isotropic. These large scales have a directional preference that is generally determined by the geometry / boundary conditions of the flow. So the RANS approach, which is rather impartial and doesn't discriminate between the scales, subjects them all to a similar treatment. So, in the scheme of things the directional dependence of the large scales gets smudged out at the price of accuracy.

A general turbulence model, which could be applied to a various set of practical problems has thus far been elusive, but, the problem of using a single turbulence model across all scales of turbulence can be addressed. The Large Eddy Simulation (LES) does just that. It is a numerical approach in which the larger scales of turbulence are solved for directly in the solution process and the smaller scales, which have a universal behaviour, are addressed in the form of a turbulence model.

Another primary variation of LES with respect to RANS lies in the absence of the concept of time averaging. LES uses a spatial filter to separate the large scales from the small ones. The spatial filter length, is generally about the same order as the spatial discretization. Upon applying the spatial filtering process on the time dependent equations, the information about the scales smaller the the fliter is lost; and only the details of the length scales to be resolved (the large ones) are retained. The interaction between the resolved and the unresolved eddies gives rise to an additional set of stresses known as the Sub Grid Scale (SGS) stresses. Its these set of stresses that are modeled using different SGS models. Further, details of the LES method used in this effort are provided in Section.3.3.

Direct Numerical Simulation In addition Eq.2.23, certain other formulations through dimensional analysis help characterize the smallest scales of turbulence also known as the Kolmogrov scales, they are given by

$$
\begin{gathered}
\eta \sim\left(\nu^{3} / \epsilon\right)^{1 / 4} \\
u_{\eta} \sim(\epsilon \nu)^{1 / 4}
\end{gathered}
$$




$$
\tau_{\eta} \sim(\nu / \epsilon)^{1 / 2}
$$

where $\eta, u_{\eta}, \tau_{\eta}$ represent the Kolmogrov length, velocity and time scales.

The ratio of the Kolmogrov scales and the larger integral $(l)$ scales can be defined as a function of a Reynolds number Re defined through the RMS of velocity fluctuation $u^{\prime}$. One can now arrive at the ratio between the largest and the smallest scales:

$$
\begin{gathered}
\eta / l \sim R e^{-3 / 4} \\
u_{\eta} / u^{\prime} \sim R e^{-1 / 4} \\
\tau_{\eta} / \tau^{\prime} \sim R e^{-1 / 2}
\end{gathered}
$$

In the context of Direct Numerical Simulation (DNS) the Navier-Stokes equations are solved for all eddy scales, down to the smallest scales of turbulence. This means that, the smallest possible spatial and temporal scales must be resolved, and thus no 'modeling' of the turbulence terms is needed. Such an approach however, does not come cheap, and there is a considerable level of computational penalty associated with it, as shall be shown.

If $h$ is the uniform grid spacing, and $\mathrm{N}$ the number of grid points, then for resolving the Large scales $L_{\max }$

$$
N h>L_{\max }
$$

And by definition of DNS to be able to resolve even the Kolmogrov scales

$$
h \leq \eta
$$

Using the limiting cases of Eq.2.50\&2.51 one would have

$$
N^{3} \sim R e^{9 / 4}
$$

which implies for a DNS, the number of grid nodes, and by extension the computational resources required, would scale as the $2.25^{\text {th }}$ power of the Reynolds Number. Therefore, a channel flow problem with an $R e=10^{6}$ requires $15 \mathrm{e} 12$ grid nodes, and would take a computer running at several giga flops around a hundred years (Hu, 2005)! The largest DNS ever executed to date has been by Lee et al. (2013), where the Reynolds Number calculated based on friction velocity was $R e_{\tau}=5200$ requiring a simulation with 242 billion degrees of freedom. 


\section{Chapter 3}

\section{Formulation of Multiphase-Flow Models}

\subsection{Hydrodynamic Forces on a rising bubble}

The problem under consideration involves a bubble "particle"; treated as a particle in the case of E-L simulation, or just another continuous media if the E-E is chosen. Regardless, the interaction between the the bubbles and the primary fluid must be set in context. These interactions are defined in the form of forces that go into the equations as source terms. The level of detail and the extent to which such forces must be considered or accounted for, in the numerical simulations, is a matter of context. In other words a decision about which forces must be accounted for and which must be neglected depends on the nature of the physics that is of interest and is the prime motivation for the modeling to begin with. What is being presented here is a summary of the kinds of hydrodynamic forces that act on a rising bubble in a liquid media. It is worth noting, that in reality, multiphase materials processes could involve regimes where the fluid under consideration would exhibit Non-Newtonian behaviour; the results here limit themselves to cases where the primary fluid is Newtonian, the Non-Newtonian case remains out of the scope of the present study. 


\subsubsection{The non-dimensional groups}

A fluid particle, in general, is subjected to deformations due to the hydrodynamics. So in order to judge the nature or the shape of the bubble certain non-dimensional numbers help characterize the shape of the bubble. Some of such non-dimensional numbers are:

The Weber Number:

$$
W e=\frac{\rho U^{2} d_{k}}{\sigma}
$$

The Eötvös Number:

$$
E_{O}=\frac{\Delta \rho \mathbf{g} d_{k}^{2}}{\sigma}
$$

The Morton number:

$$
M o=\frac{\mathbf{g} \Delta \rho \mu^{4}}{\rho^{2} \sigma^{3}}
$$

where $d_{k}$ is the particle diameter, $\Delta \rho=\left|\rho-\rho_{k}\right|$ or the difference between the densities of the main phase and the particle phase, and $\sigma$ is surface tension. In other words Weber number gives the ratio between the inertial forces and the force induced by surface tension, Eötvös Number gives the ratio between the forces of buoyancy and surface tension force. It is also to be noted that the Morton number contains only fluid properties and the gravitational acceleration, becomes a characteristic of the set of fluids under consideration.

Another interesting inference that could be made from the definition of the Weber number is that for a bubble to be spherical :

$$
W e \ll 1
$$

which implies that a bubble tends to become a natural sphere when the surface tension force is much larger than the forces that tend to deform it.

It then becomes imperative to check these criteria for gas-fluid flows before an assumption of sphericity is made for the gas bubbles. Next, we consider other parameters: 
The Particle Reynolds Number:

$$
R e_{p}=\frac{\left|\mathbf{V}_{r e l}\right| d_{k}}{\nu}
$$

where $V_{\text {rel }}$ is the relative velocity between the dispersed and the continuous phase. The particle Reynolds number is the ratio of the particle inertia force and the fluid viscous force on particle. This Reynolds Number is based on the magnitude of the velocity difference between the two phases, and gives about the flow around the particle.

\section{The Stokes Number :}

$$
S t=\frac{\tau_{k}}{\tau}
$$

where, $\tau$ is the characteristic flow time and $\tau_{k}$ is the momentum response or the relaxation time of the particle, given by

$$
\tau_{k}=\frac{\rho_{k} d_{k}^{2}}{18 \mu}
$$

This gives the ratio between the particle response time and some time scale of the main flow. In other words this non dimensional number gives an estimate of the extant to which the particle is affected by the carrier phase. For example if $S t \ll 1$ then $\tau_{k} \ll \tau$ which implies that the particle has had ample time to reach its terminal velocity, before there was an appreciable change in the ambient carrier fluid velocity. In this case the particle then behaves as a mere passive trace of the carrier velocity itself; and subjects itself completely to the whims of the velocity field around it, and the local velocity of the two phases will be nearly equal. If we consider the other case when $S t \gg 1$ and $\tau_{k} \gg \tau$, here the particle is still accelerating and has not had enough time to attain its terminal velocity. So in this case the particle tends to not get disturbed by subtle changes in the velocity field around it, and is said to be governed by the mean convection and gravity alone $(\mathrm{Hu}, 2005)$. From another point of view, the particle time is too slow to respond to the rapid fluctuations of the flow turbulence. 
The Froude Number:

$$
F r=\frac{\left|\mathbf{V}_{r e l}\right|^{2}}{\mathbf{g} l}
$$

Where $l$ is the characteristic length scale of the carrier fluid.

This gives a measure of the ratio of the inertial forces and those of the convection. In a bubble column however, it could also represent the likelihood of a bubble to be entrapped in a vortex. This is done by relating the vortical entrapment velocity to the terminal rise velocity (Rightley, 1995).

\section{The Length Scale Ratio:}

$$
\gamma_{l}=\frac{d_{k}}{l}
$$

The length scale ration, $\gamma_{l}$ gives a measure of the particle size relative to the scales of the flow surrounding it. Now, if 1 is supposed to be the Kolmogrov Scale; i.e the smallest scale of turbulence that can manifest itself, then if $\gamma_{l} \ll 1$ implying $d_{k} \ll l$, translates to a scenario where the system is considered highly dispersed , and the particle could then be considered at the mercy of the flow around it; in other words the flow of the particle is governed by the flow field around it.

\subsubsection{The Forces}

For the sake of better understanding and simplicity, a point volume approach for the particle is considered in the discussion that follows. It must be noted here that, such a treatment could be extended to a resolved volume approach or the Eulerian-Eulerian approach with subtle changes and without loss of generality. The motivation for this section is to purely dwell on the physics of the forces rather than their translations to E-E frames of reference.

The point volume approach implies that the motion of a particle in the fluid media could be encompassed in the form of an Ordinary Differential Equation

$$
m_{p} \frac{d v}{d t}=\mathbf{F}_{p}
$$


The expression on the right hand side represents the vector sum of all the forces that act on the particle. The forces (both body and surface) playing a major role are discussed below.

\section{The Virtual Mass Force}

The forces on the particle, in general, are measured in a steady state; when there is no relative acceleration between the particle and the continuous media. However when the particles (bubbles or droplets) do accelerate in the medium with respect to the surrounding fluid, then there are certain inertial forces that the continuous media exerts on the particle that must be accounted for. As a result of such an inertial formulation, there could then be said to be an apparent increase in the mass of the particle compared to its original mass. This increase is termed as the Virtual Mass and the force that cause it is termed as Virtual Mass Force or Added Mass Force. (Drew \& Lahey, 1993) In other words when a particle accelerates through the system, then there is a corresponding acceleration in the surrounding fluid. This acceleration of the fluid, then tends to do some work on the particle itself, by virtue of the Newton's 3rd Law . This is in the form of a transient force acting in a direction opposite to that of the motion of the particle; resulting in an apparent increase in mass of the particle.

With further discussion it will be seen that this Virutual Mass Force could be neglected when the density of the particle is less, compared to the density of the fluid surrounding it. However, if that is not the case then it becomes of paramount importance to take this force into consideration. The Virtual Mass Force is given by:

$$
\mathbf{F}_{v m}=-\frac{1}{2} m_{f} \frac{d}{d t}\left(\mathbf{v}_{k}-\mathbf{v}\right)
$$

Where $m_{f}$ represents the mass of the fluid with a volume equal to that of the particle. Therefore $m_{f}=\alpha_{k} \times \rho$; it is also to be noted that the constant $\frac{1}{2}$ is termed as the Added Mass Coefficient $\left(C_{A}\right)$, and the value is obtained empirically for a creep flow over a rigid sphere. 


\section{The Forces due to Gravity}

The net force of gravity experienced by the particle, or the buoyant force is given by :

$$
\mathbf{F}_{G}=\left(\rho_{k}-\rho\right) \mathbf{g} \forall_{p}
$$

Where $\forall_{p}$ represents the volume of the particle.

\section{The Drag Force}

The steady state, drag force is defined by the equation given by:

$$
\mathbf{F}_{\text {drag }}=-\frac{1}{2} C_{D} \rho A_{f r}\left|\mathbf{V}_{k}-\mathbf{V}_{p}\right|\left(\mathbf{V}_{k}-\mathbf{V}_{p}\right)
$$

The $C_{D}$ mentioned above is the coefficient of drag and is obtained empirically. The value of $C_{D}$ manifests itself as a function of the particle Reynolds number $R e_{k}$, so a generic formulation for the coefficient of drag is given as :

$$
C_{D}=\frac{24}{R e_{p}} f\left(R e_{p}\right)
$$

where $\mathrm{f}$ is termed as a the drag factor; which is a function of the particle reynolds number, which is given by (3.5).

\section{History Force}

This force is known as the Basset Force. This was derived by (Basset, 1888) and earlier by (Boussinesq, 1885). This force is also an unsteady force like the Virtual Mass Force, and is defined as the force that accounts for the viscous effects of the temporal delay in boundary development, due to the change in the relative velocity with time.

A better understanding of the history force could be attained through the solution of the Stoke's First Problem (White, 2006), which is the case of an infinitely long flat plate below a viscous fluid subjected to sudden step velocity change from zero to $u_{0}$. For low reynolds case of such a flow, the Navier-Stokes equation get reduced to the form

$$
\frac{\partial u}{\partial t}=\nu \frac{\partial^{2} u}{\partial x}
$$


The analytical solution of Eq.3.15 is

$$
u=u_{0}-u_{0} \operatorname{erf}\left(\frac{y}{2 \sqrt{\nu t}}\right)
$$

where, $\operatorname{erf}(x)$ is the error function defined by

$$
\operatorname{erf}(x)=\frac{2}{\sqrt{\pi}} \int_{0}^{x} e^{-t^{2}} \mathrm{~d} t
$$

The shear stress at the plate given by this profile would be

$$
\tau=u_{0} \sqrt{\frac{\rho \mu}{\pi}}
$$

The cumulative effect of this stress is given by

\section{Fluid-stress Force}

The fluid stress force $\mathbf{F}_{S}$ is the force due to the undisturbed flow. In other words, it would be the force acting on the particle, if it were instead a fluid element. Since static pressure has already been considered in the buoyancy term $\mathbf{F}_{G}$, only the dynamic pressure would be considered. The expression for the fluid stress force is given by

$$
\mathbf{F}_{S}=m_{f} \frac{D \mathbf{u}}{D t}
$$

\section{Wall Effect}

The interaction between the particle and the wall is important for wall bounded flows. The effect of the wall is generally taken into account by modifying the lift and drag coefficients or through and introduction of an additional wall force $F_{W}$.

The presence of boundaries (Fig. 3.1), such as walls causes changes in the flow pattern of the bubbles and the overall nature of the plume. The bubbles, which could be considered as submerged particles tend to get repelled by the wall due to the wall shear induced lift, which is the lift induced to the near wall velocity gradients on either side of the particle. Moreover, the presence of a boundary introduces an asymmetry in an otherwise symmetric flow, which in turn could cause a torque setting the particle into rotation, thus enhancing the lateral component of the force. These effects are found to be present in lower and higher Reynolds number regimes. The presence of these particles also tends to effect the stability of the 


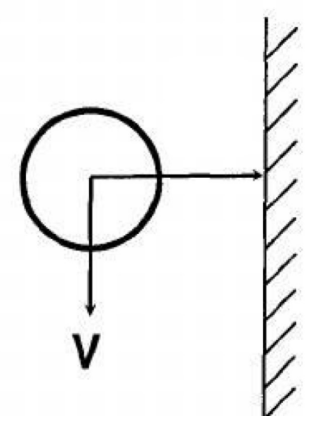

Figure 3.1: Particle near wall

boundary layer and the transport processed occurring in the region (Michaelides, 2006).

A second important near wall effect, is the Coanda Effect which becomes very significant in asymmetrically aerated columns. As shown in 3.2 when a jet nears

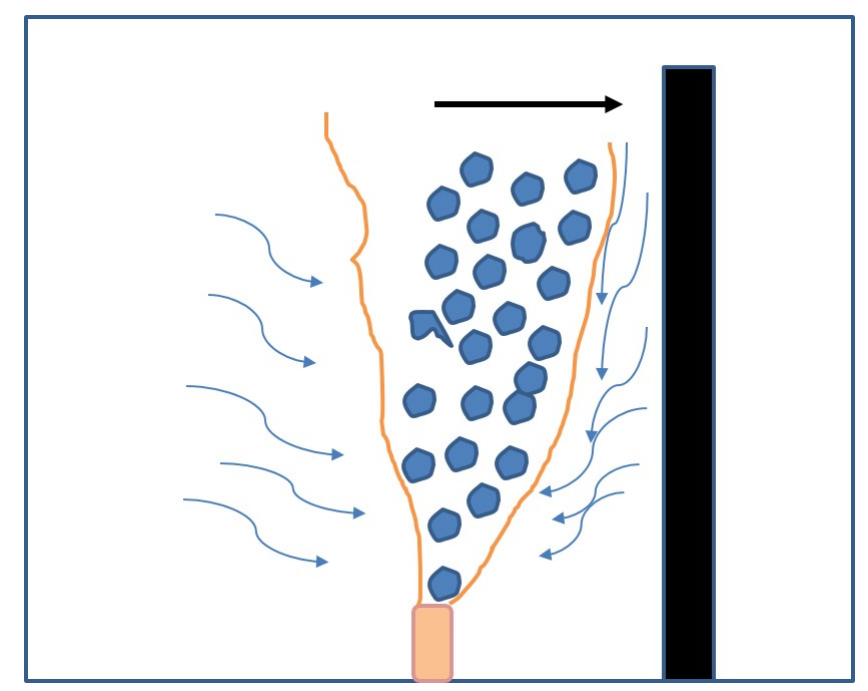

Figure 3.2: The Coanda effect

the surface (wall), it tends to restrict the entrainment in the region as opposed to the other side of the plume where the entrainment remains unrestricted. Therefore the flow in the restricted region must accelerate in order to try and balance the momentum transfer, this results in a drop in the pressure. This pressure difference then deflects the jets towards the wall, and the jet tends to eventually attach itself 
to it. Such an attachment remains even if the wall were to curve away from its initial direction. The reader is directed to $(\mathrm{Hu}, 2005)$ for a detailed discussion, on the formulation of the $F_{W}$ term.

\section{Lift Force}

The lift force acting on a spherical particle is of two kinds the Saffman Lift Force due to the velocity gradient and the Magnus Lift Force, which is due to the rotation of the particle. So, both the effects of shear and the rotation of the particle can cause an uneven pressure distribution around it, thus resulting in a net lift.

The Magnus lift force, along with an empirical lift coefficient $C_{L, \text { mag }}$, is given by (Crowe et al. , 1998)

$$
\mathbf{F}_{\text {mag }}=-\frac{1}{2} C_{L, M a g} \rho A_{f r}\left|\mathbf{V}_{\mathbf{k}}-\mathbf{V}_{\mathbf{p}}\right|\left(\mathbf{V}_{\mathbf{k}}-\mathbf{V}_{\mathbf{p}}\right) \times \frac{\omega_{\text {rel }}}{\left|\omega_{\text {rel }}\right|}
$$

where

$$
\omega_{\text {rel }}=\omega_{\mathbf{p}}-0.5 \omega
$$

and $\omega_{\mathbf{p}}$ is the particle angular velocity and $\omega$ is the vorticity of the flow given by

$$
\omega=\Delta \times \mathbf{V}_{\mathbf{k}}
$$

The presrciption for $C_{L, \text { mag }}$ is given by

$$
C_{L, \operatorname{mag}}=\min (R o / 4,0.5)
$$

Where Ro is the a rotation parameter termed as the Rosby Number

$$
R o=\frac{d_{p}\left|\omega_{\text {rel }}\right|}{\left|\mathbf{V}_{\mathbf{k}}-\mathbf{V}_{\mathbf{p}}\right|}
$$

In similar terms the Saffman lift force could be defined as

$$
\mathbf{F}_{\text {Saff }}=-\frac{1}{2} C_{L, \text { Saff }} \rho A_{f r}\left|\mathbf{V}_{\mathbf{k}}-\mathbf{V}_{\mathbf{p}}\right|^{2} \frac{\left(\mathbf{V}_{\mathbf{k}}-\mathbf{V}_{\mathbf{p}}\right) \times \omega}{\left|\left(\mathbf{V}_{\mathbf{k}}-\mathbf{V}_{\mathbf{p}}\right) \times \omega\right|}
$$

Where $C_{L, s a f f}$ is given empirically as

$$
C_{L, s a f f}=0.5 R o^{0.25}
$$




\subsection{Eulerian Multiphase-Flow Models}

The Eulerian-Eulerian model considers the gas and liquid phases both in an Eulerian reference frame. Both phases are treated as a continuous and an interpenetrating media. The main difference between this approach and the E-L approach lies in the treatment of the dispersed phase; the E-L approach treats the dispersed phase as discrete particles that must be tracked where as E-L approach treats it as continuous.

The nature of the physics involved requires an interaction between the phases in the form of forces. Such an interaction must then be provisioned for in the numerical models, regardless of the approach. There are, as shall be seen, differences in the methods to numerically accommodate such forces; but those will only be that of detail and not of essence. The E-E approach is obtained through the inclusion of certain inter-phase interaction terms in the individual solutions of the mass and momentum balance equations (Mudde \& Simonin, 1999). In the case of the E-L approach, a separate force balance equation is solved for each bubble while the continuous phase interacts with these bubbles through a source term in the momentum equation. It could be then said that the E-L model finds itself limited by the number of bubbles (particles) it can track at a given time and that makes it more expensive computationally. However, the E-L model makes the accounting for the break-up and collisions of the particles through the inclusion of some numerical models, relatively easy. The E-E approaches are applicable across a wider range of volume fractions when compared to the E-L approach (Diaz et al. , 2008). It is important to note that, in the current study the particle breakup and coalescence are not accounted for and is left for further study.

This Eulerian treatment for the dispersed phase can be done in two ways; the mixture model and the two-fluid model.

\subsubsection{The Mixture Model}

The mixture model could be considered a simplified multiphase model where a single N-S equation is solved for the entire fluid consisting of different phases. The 
phases could be having different velocities, but assume a local equilibrium. The mixture models can also be used to calculate Non-Newtonian viscosity. (ANSYSFLUENT, n.d.) . The mixture model can accommodate multiple phases (fluids or particles) by solving the momentum, continuity and energy equations for the entire mixture taken at once. This approach can be said to be at an advantage when the nature of interaction between the phases is not completely known or of redoubtable nature, and/or when there is a wide distribution of the particulate phase. It can be then, said to be a compromise from a full blown E-E simulation where the N-S equations are solved for both the phases. What then is the catch? The trade off? These are well highlighted by (Landau \& Lifschitz, 1971), in which they state the N-S equations could be applied to a two-phase flow if two conditions are met : (i) If the dispersed phase is smaller in size compared to the geometry of the main flow field. (ii) It does not change the overall fluid density by a notable amount. By subscribing to the above conditions one has agreed to the flow being a mixture of the two phases, the fluid density $\rho_{m}$ now representing the mixture density, the viscosity $\mu_{m}$ now representing the effective viscosity of the mixture. When the inertia of the dispersed phase is not to be neglected (the phases moving at different velocities), an additional formulation called the slip velocity is added to the set of equations, based largely on the Stokes number. These set of equations are then closed by a transport equation for the volume fractions of the secondary cases.

The set of governing equations that need to be addressed numerically for this model can be summarised as follows:

The continuity equation for the mixture:

$$
\frac{\partial}{\partial t}\left(\rho_{m}\right)+\nabla \cdot\left(\rho_{m} V_{m}\right)=0
$$

where $V_{m}$ is the mass-averaged velocity:

$$
V_{m}=\frac{\sum_{k=1}^{n} \alpha_{k} \rho_{k} V_{k}}{\rho_{m}}
$$


and $\rho_{m}$ is the mixture density

$$
\rho_{m}=\sum_{k=1}^{n} \alpha_{k} \rho_{k}
$$

and $\alpha_{k}$ is the volume fraction of phase $\mathrm{K}$.

The momentum equation for the mixture is obtained by the sum of the momentum equations for all phases.

$\frac{\partial}{\partial t}\left(\rho_{m}\left(\mathbf{V}_{m}\right)+\nabla \cdot\left(\rho_{m}\left(\mathbf{V}_{m}\right)\left(\mathbf{V}_{m}\right)\right)=\nabla p+\nabla \cdot\left[\mu_{m}\left(\nabla \mathbf{V}_{m}+\nabla \mathbf{V}_{m}^{T}\right)\right]+\rho_{m}+\mathbf{F}+\zeta_{\text {slip }}\right.$

where $n$ is the number of phases, $\mathbf{F}$ is a body force.

$\mu_{m}$ ss the viscosity of the mixture given by :

$$
\mu_{m}=\sum_{k=1}^{n} \alpha_{k} \mu_{k}
$$

$\zeta_{\text {slip }}$ could be termed as the sum of the inertial forces of the dispersed phases arising due to a slip velocity with respect to the main phase:

$$
\zeta_{\text {slip }}=\nabla \cdot\left(\sum_{k=1}^{n} \alpha_{k} \rho_{k} \mathbf{V}_{\text {slip }, k} \mathbf{V}_{\text {slip }, k}\right)
$$

where

$$
\mathbf{V}_{s l i p, k}=\mathbf{V}_{k}-\mathbf{V}_{m}
$$

As mentioned earlier, these set of equations need to be closed with an additional formulation that would account for the volume fraction term $\alpha_{k}$; such a formulation is found in a set of continuity equations for each secondary phase $k$ (ANSYS-FLUENT, n.d.)

$$
\frac{\partial}{\partial t}\left(\alpha_{k} \rho_{k} \mathbf{V}_{k}\right)=-\nabla \cdot\left(\alpha_{k} \rho_{k} \mathbf{V}_{s l i p, k}\right)+\sum_{q=1}^{n}\left(\grave{m}_{q k}-\grave{m}_{k q}\right)
$$

where the last term on the right hand side $\left(\grave{m}_{q p}-\grave{m}_{p q}\right)$ represents the interphase mass transfer which accounts for phase changes. 


\subsubsection{Multi Fluid Eulerian Model}

In this approach the two phases are assumed to be interpenetrating continua as well; but the kinetics of every phase is recognized in its own right. This could then be considered as a full blown approach where the volume averaged N-S equations are solved for each phase, and the interaction between the phases is attained through inclusion of interphase exchange terms. In the current effort the commercial software ANSYS FLUENT was used for this approach and the following are the equations addressed by the solver (ANSYS-FLUENT, n.d.)

The conservation of momentum for a phase $\mathrm{k}$ is given by

$$
\begin{gathered}
\frac{\partial}{\partial t}\left(\alpha_{k} \rho_{k}\left(\mathbf{V}_{k}\right)+\nabla \cdot\left(\alpha_{k} \rho_{k}\left(\mathbf{V}_{k}\right)\left(\mathbf{V}_{k}\right)\right)=-\alpha_{k} \nabla p+\nabla \cdot \overline{\bar{\tau}} \alpha_{k} \rho_{k} \mathbf{g}+\right. \\
\sum_{q=1}^{n}\left(K_{q k}\left(\mathbf{V}_{q}-\mathbf{V}_{k}\right)+\grave{m}_{q k} \mathbf{V}_{q k}-\grave{m}_{k q} \mathbf{V}_{k q}\right)+\left(\mathbf{F}_{k}+\mathbf{F}_{l i f t, k}+\mathbf{F}_{v m, k}\right)
\end{gathered}
$$

Where $\mathbf{g}$ is acceleration due to gravity, $\mathbf{F}_{k}$ is an external body force, $\mathbf{F}_{l i f t, k}$ is the lift force and $\mathbf{F}_{v m_{k}}$ is the virtual mass force.

\section{Virtual Mass Force}

This force has been explained in detail in the Section 3.1.2; but the treatment by (ANSYS-FLUENT, n.d.) is discussed here. When a secondary phase $\mathrm{k}$ accelerated relative to the primary phase $\mathrm{p}$; then the inertia of the primary-phase mass encountered by the accelerating bubbles exerts a "Virtual Mass Force" on the bubbles

$$
\mathbf{F}_{v m}=0.5 \alpha_{k} \rho_{q}\left(\frac{d_{p} \mathbf{V}_{q}}{d t}-\frac{d_{k} \mathbf{V}_{k}}{d t}\right)
$$

where the term $\frac{d_{q}}{d t}$ represents the material derivative of the form

$$
\frac{d_{q} \phi}{d t}=\frac{\partial(\phi)}{\partial t}+\left(\mathbf{V}_{p} . \nabla\right) \phi
$$

It is to be noted that as the virtual mass force is added to the right hand side of the momentum equation for both the phases (the primary as well as secondary) and that implies $\mathbf{F}_{v m, p}=-\mathbf{F}_{v m, k}$. This force becomes significant when the phase density of the secondary phase is much smaller than that of the primary phase. 


\section{Lift force}

The formulation for the lift forces has not been in included in the current effort. It is observed in literature by (Diaz et al. , 2008) that the inclusion of the lift forces doesn't have major impact on the the solution; especially since the influence of the lift force is small compared to that of the drag force (ANSYS-FLUENT, n.d.). Therefore it is recommended to not include this term if the particle (bubble) size is relatively small.

The formulation is as follows :

$$
\mathbf{F}_{l i f t}=-C_{l} \rho_{k} \alpha q\left(\mathbf{V}_{k}-\mathbf{V}_{q}\right) \times\left(\nabla \times \mathbf{V}_{k}\right)
$$

and since this term is added on the right hand side of the momentum equation for each phase $\left(\mathbf{F}_{l i f t, p}=\mathbf{F}_{l i f t, k}\right)$

\section{The interphase exchange / drag force}

The interaction between the primary and the secondary phase is modelled through the interphase exchange coefficient. A discussion on the drag force has been provided in detail in Section 3.1.2. The exchange coefficient is written in the general form

$$
K_{k q}=\frac{\alpha_{k} \alpha_{q} \rho_{k} f}{\tau_{k}}
$$

where $\mathrm{f}$ is the drag function that will be contextual depending on the model chosen, which shall be further discussed. $\tau_{k}$ represents the particle relaxation time.

$$
\tau_{k}=\frac{\rho_{k} d_{k}^{2}}{18 \mu_{k}}
$$

where $d_{k}$ is the diameter of the bubbles or droplets of phase $\mathrm{k}$.

The drag law :

The drag law plays a predominant role in getting in modelling the physics of the bubble column. It is worth noting that in the current effort the Schiller-Naumann drag law yielded results that were not concurring with the experimental values. Hence, the drag law propounded by (Grace et al. , 1976) was used; and with success. Hence it could then be stated that the numerical results were found to be very sensitive to the formulation of the drag forces between the bubbles and the liquid. Since the aforementioned drag law was not found in the ANSYS-FLUENT 
library, a user defined function was written which can be found the Appendix A. This user defined function helps customize the interphase exchange coefficient $K_{k} p$ and supply it to the solver. The coefficient according to this law is defined as:

$$
K_{k q}=\frac{3}{4} C_{D} \frac{\alpha_{k} \alpha_{q} \rho_{q}\left|\mathbf{V}_{k}-\mathbf{V}_{q}\right|}{d_{k}}
$$

where $\alpha_{k} \alpha_{p}$ are volume fractions of the gas and liquid phase respectively, $\rho_{q}$ is the density of the liquid phase. and $C_{D}$ is the coefficient of drag which is given by (Grace et al. , 1976) as

$$
C_{D}=\frac{4}{3}\left(\frac{\rho_{q}-\rho_{k}}{\rho_{q}}\right) \frac{\mathbf{g} d_{k}}{\mathbf{V}_{b}^{2}}
$$

where $V_{b}$ is defined as the terminal velocity of the bubble which is calculated as

$$
V_{b}=\frac{\mu_{p}}{\rho_{q} d_{k}} M^{-0.149}(J-0.857)
$$

where $\mathrm{M}$ is morton number and $\mathrm{J}$ is

$$
J= \begin{cases}.94 H^{.751}, & \text { if } 2<H \leq 59.3 \\ 3.42 H^{0.441}, & \text { if } H>59.3\end{cases}
$$

Where

$$
H=\frac{4}{3} E o M^{-0.149}\left(\frac{\mu_{l}}{\mu_{\text {ref }}}\right)^{-0.14}
$$

Eo being the Eötvös number and $\mu_{\text {ref }}=0.0009 \mathrm{kgm}^{-1} S^{-1}$

\subsection{The Eulerian Lagrangian Method}

The primary way that this method shall differ from the aforementioned one, is in its treatment of the dispersed phase (air). Contrary to the E-E approach the assumption of continuum is no longer made for the dispersed phase, and instead is subjected to a particular treatment; particles whose dynamics and trajectories are then governed by the Newton's Second law. The continuum treatment for the primary phase still holds good in this approach, and the Navier-Stokes equations for it are solved for. The two coupling between the continuous and the dispersed phase still needs to be addressed, and is done in a fashion similar to that of the E-E method; through the inclusion of additional source terms on the right hand 
side of the momentum equation, for the continuous phase, and the force balance equation, for the dispersed phase. Without the forward (continuous to dispersed) and the backward (dispersed to continuous), the system would not be set into motion.

The present effort assumes the validity of the single phase Large Eddy Simulation(LES) equations (Hu \& Celik, 2008), with the reverse coupling achieved through the PSI-ball method .(Hu, 2005) The premise of which lies in the assumption that the interphase force the dispersed particle shall redistribute to the Eulerian field of the primary phase, will only be limited to those nodes of the Eulerian field that lie in the predefined influence radius; hence the name PSI-ball. In the present effort the diameter of the sphere of influence, D, is formulated as thus :

$$
D=\max \left(2 d_{p}, 2 h_{\max }\right)
$$

where $d_{p}$ is the particle diameter and $h_{\max }$ is the max grid spacing. A truncated Gaussian distribution with a zero mean and a variance $\sigma=2 D$ is used to formulated the distribution function g given as :

$$
\int_{\Omega \text { ball }} g\left(\xi-\mathbf{y}_{\mathbf{n}}\right) d \Omega=1
$$

where $\Omega_{\text {ball }}$ represents the region inside the influence sphere $\mathbf{y}_{N}$ is the location of the the particle $n$. If $\mathbf{F}_{n}$ is the force exerted by a particle $n$, then the total interphase momentum transfer experienced by a cell $j$ from $\mathrm{N}$ such particles, $\mathbf{F}_{j}$, is given by

$$
\mathbf{F}_{j}=\frac{1}{\forall_{j}} \sum_{n=1}^{N} \mathbf{F}_{n} \int_{\Omega_{j}} g\left(\xi-\mathbf{y}_{\mathbf{n}}\right) d \Omega
$$

It must be noted that the expression for $\mathbf{F}_{j}$ is in per unit volume form; where $V_{j}$ is the volume of the cell $j$.

The filtered N-S equations for the LES, with backward coupling are given by:

$$
\begin{gathered}
\frac{\partial \bar{u}_{j}}{\partial x_{j}}=0 \\
\frac{\partial}{\partial t}\left(\rho \bar{u}_{i}\right)+\frac{\partial}{\partial x_{j}}\left(\rho \bar{u}_{j} \bar{u}_{i}\right)=-\frac{\partial \bar{p}}{\partial x_{i}}+\frac{\partial}{\partial x_{j}}\left(\mu \frac{\partial \bar{u}_{j}}{\partial x_{j}}\right)-\frac{\partial \tau_{i j}^{r}}{\partial x_{j}}+\mathbf{F}_{j}
\end{gathered}
$$


Here the overbar indicates the filtered quantity, it is of interest to note the $\tau_{i j}^{r}$ is the part of the residual stress which represents the Sub Grid Scale (SGS) effect, and has to be modeled. The SGS turbulence generated by the dispersed(particulate) phase is considered negligible, and hence the modeling of the subgrid viscosity is limited to primary phase alone. The standard Smagorinsky model is used (Smagorinsky, 1963) :

$$
\tau_{i j}^{r}=-2 \rho \nu_{t} \overline{S_{i j}}
$$

where

$$
\nu_{t}=l_{s}^{2} \bar{S}, l_{S}=C_{S} \Delta, \bar{S}_{i j}=\frac{1}{2}\left(\frac{\partial \bar{u}_{i}}{\partial x_{j}}+\frac{\partial \overline{u_{j}}}{\partial x_{i}}\right), \bar{S}=\left(2 \overline{S_{i j}} \overline{S_{i j}}\right)^{1 / 2}
$$

Where $C_{s}$ is the Smagorinsky constant and $\Delta$ the filter width. For a detailed investigation of the efficacy and veracity of this method the reader is directed to the work by $(\mathrm{Hu}, 2005)$

In contrast to the RANS(Reynolds Averaged Navier Stokes) approach used for the E-E part of the simulation, for E-L part LES has been used. LES only resolved large structures of the liquid through a filtering operation on the Navier-Stokes equations. The main difference then lies in the spectrum of turbulence that gets modeled, with the RANS approach going for the entire spectrum and LES modeling only the high wave number part (Hu \& Celik, 2008).

\section{Forces on the particle:}

The motion of the particle is governed by the following equation which is a respresentation of Newton's second law:

$$
m_{P} \frac{d \mathbf{V}}{d t}=\mathbf{F}_{p}
$$

Where $\mathbf{F}_{p}$ is the sum of forces acting on the particle from the primary phase, being considered in the computation are given by

$$
\mathbf{F}_{p}=\mathbf{F}_{G}+\mathbf{F}_{D}+\mathbf{F}_{L}+\mathbf{F}_{S}+\mathbf{F}_{A}
$$

Where $\mathbf{F}_{G}$ is the buouyancy force, $\mathbf{F}_{D}$ the steady state drag, $\mathbf{F}_{S}$ the fluid stress, $\mathbf{F}_{L}$ lift, $\mathbf{F}_{H}$ history force, and $\mathbf{F}_{A}$ the added mass force. An indepth review of 
these analysis of these forces has been provided by (Hu, 2005).

The drag force formulation, is taken from (Clift et al. , 1988)

$$
C_{D}= \begin{cases}\frac{24}{R e_{p}}\left(1+0.15 R e_{p}^{.687}\right), & \text { if } R e_{p} \leq 800 \\ 0.44, & \text { if } R e_{p}>800\end{cases}
$$

The value of coefficient of lift $C_{L}=0.53$. 


\section{Chapter 4}

\section{Results and Discussion}

The plume oscillation period, in its most general sense, could be considered to be a good parameter to characterize the overall nature and regimes of centrally aerated bubble columns. This claim is further reinforced by noting that the plume oscillation period (POP) represents the most dominant frequency existing in the system. In other words, it could be seen as a measure of the frequency at which the system repeats itself at large, within a window of certain nominal departure. This then, by extension could be said to also characterize mixing obtained in the system. It is with this inference in mind, that the efficacy of the aforementioned numerical formulations shall now be determined. The objective of the effort is not to prescribe a clear winner and the right way forward, but to understand and analyze the merits and limitations that affiliate themselves to their respective numerical approaches.

\subsection{Eulerian-Eulerian Simulations}

The numerical simulations were based on the experimental setup of (Diaz et al. , 2008), which included a $0.20 \mathrm{~m}$ wide $0.04 \mathrm{~m}$ deep and $0.45 \mathrm{~m}$ high liquid column Fig. 4.1. The inlet included an aluminum distributor with 8 centered holes of 1 $\mathrm{mm}$ diameter and $6 \mathrm{~mm}$ pitch. The superficial gas velocity $U_{g}$ was then varied to study the different regimes of flow (Díaz et al. , 2008).

The spatial resolution for the E-E simulations was picked in accord with the coarse 
grid used in (Diaz et al. , 2008), 17(w) x 7(d) x 25(h). Time steps of $0.01 \mathrm{~s}$ and $0.005 \mathrm{~s}$ were used to study the effect of temporal resolution. The inlet region has been modeled as a velocity inlet in the form of a rectangle with an area equal to the total area enclosed by the holes. Only bubbles with a diameter of $5.5 \mathrm{~mm}$ are considered to be entering the system. The computational domain itself is actually larger than the one used by (Diaz et al. , 2008), in this case the height of the system is assumed to be larger than the bed height of water. So the actual computational domain is $17(\mathrm{w}) \times 7(\mathrm{~d}) \times 30(\mathrm{~h})$, with water filled up till $0.45 \mathrm{~m}$. Hence, no degassing boundary condition is used at the top, instead a pressure outlet is used.

The bubble coalescence and breakup have not been considered in this effort, hence the results seen in this case would be that of a 'mono dispersed' case. Only bubbles of diameter of $5.5 \mathrm{~mm}$ are considered in this simulation, and the idea is to see if the experimental results could be replicated. In existing literature extensive work has been done by (Becker et al. , 1994) and (Pfleger et al. , 1999), which conclude that 3D simulations of the well known $\kappa-\epsilon$ turbulence model for the continuous phase, are essential to reproduce the periodic behaviour of the bubble column in the numerical domain. It was also reported by (Sokolichin \& Eigenberger, 1999) that the use of a 2D model for modeling a flat bubble column reactor, results in the overestimation of the turbulent viscosity. This overestimation could be a result of ignoring the damping effect that the front and back walls have on the turbulent kinetic energy profile. Even in the present effort, simulations in a 2D model were attempted; and the results showed a stark absence of the desired/expected unsteady behaviour. In other words the plume was seen to remain steady and not oscillate. The need for a 3D model and the $k-\epsilon$ turbulence model then, is well established.

It has been reported by (Oey et al. , 2003) that usage of improper discretization schemes for the advective terms results in a numerical diffusion, that also results in a failure to capture the inherent transience of the physics by the calculations.

It is also worth noting here, that the information concerning the bubble diameter (in this case $5.5 \mathrm{~mm}$ ) is a part of the problem definition and not part of the solu- 
tion. What this means then, is that one has to "guess" wisely the bubble diameter for a given flow rate, as opposed to the diameter of the bubble being determined through the flow rate that is being imposed at the inlet. Such an estimate, is only feasible through some apriori knowledge of the system and its behaviour, that working hypothesis shall remain consistent throughout this effort.

The variation of the plume oscillation period (POP) with an increase in the superficial gas velocity, is studied here. The superficial gas velocity $\left(U_{G S}\right)$, is defined as the mass flow rate of the throughput of air divided by the cross-sectional area. The set of $U_{G S}$ values used here are same as that of (Diaz et al. , 2008). Table 4.1 provides the values for the actual inlet velocities:

Table 4.1: Inlet velocity values for a given superficial gas velocity.

\begin{tabular}{|c|c|}
\hline$\left.U_{G S} \mathbf{( m / s e c}\right)$ & Inlet Velocity $(\mathbf{m} / \mathbf{s e c})$ \\
\hline 0.0024 & 0.1778 \\
\hline 0.0071 & 0.5259 \\
\hline 0.0120 & 0.8889 \\
\hline 0.0166 & 1.2296 \\
\hline 0.0213 & 1.5778 \\
\hline
\end{tabular}

The discretization schemes used for the momentum equation and the equation for the volume fraction equation is the Quadratic Upwind Scheme for Convection Kinetics (QUICK). First Order Upwind has been used for the turbulence parameters whereas the pressure velocity coupling is resolved using the Semi-Implicit Method for Pressure Linked Equations (SIMPLE) (Patankar \& Spalding, 1972). A summary of the numerical schemes used for obtaining the solution is provided in Table.4.2, and details concerning the turblulence models is provided in Table. 4.3 .

The quantification of the results and the system is through horizontal liquid velocities measured at a point (monitor point) slightly offset from the vertical centre. The calculation of the plume oscillation period, was based on the velocity signal received at the aforementioned monitor point; from which for lower values of $U_{G S}$ 
Table 4.2: Solutions methods, spatial and temporal discretizations

\begin{tabular}{|c|c|}
\hline PROPERTY & SCHEME \\
\hline Pressure-Velocity Coupling & Phase Coupled-SIMPLE \\
\hline Momentum & QUICK \\
\hline Volume Fraction & QUICK \\
\hline Turbulent Kinetic Energy & First Order Upwind \\
\hline Turbulent Dissipation Rate & First Order Upwind \\
\hline Transience & First Order Implicit \\
\hline
\end{tabular}

Table 4.3: Turbulence Model Parameters

\begin{tabular}{|c|c|}
\hline Model & Standard k- $\epsilon$ \\
\hline Wall Function & Standard Wall Function \\
\hline Multiphase Turbuence Model & Dispersed \\
\hline$C_{\mu}$ & 0.09 \\
\hline$C_{1 \epsilon}$ & 1.44 \\
\hline$C_{2 \epsilon}$ & 1.92 \\
\hline$C_{3 \epsilon}$ & 1.3 \\
\hline
\end{tabular}

the periodicity is discernible. However, at higher $U_{G S}$ values distinguishing periodicity becomes difficult because of the superimposition of many harmonics, thus there occurs a need to transform the data from the time domain to the frequency domain (Darmana et al. , 2007). This transformation is performed through fast Fourier transform, and the resulting plot for the power spectral density (PSD) showcases the dominant frequency of the system. The comparison of the PSD plots with (Diaz et al. , 2008) is provided in Fig. 4.2, and a reasonable agreement is noted. It then follows that the reciprocal of the dominant frequency gives the plume oscillation period. Furthermore, as expected the characteristic frequency moves rightwards (increases) with an increase in superficial velocity. 


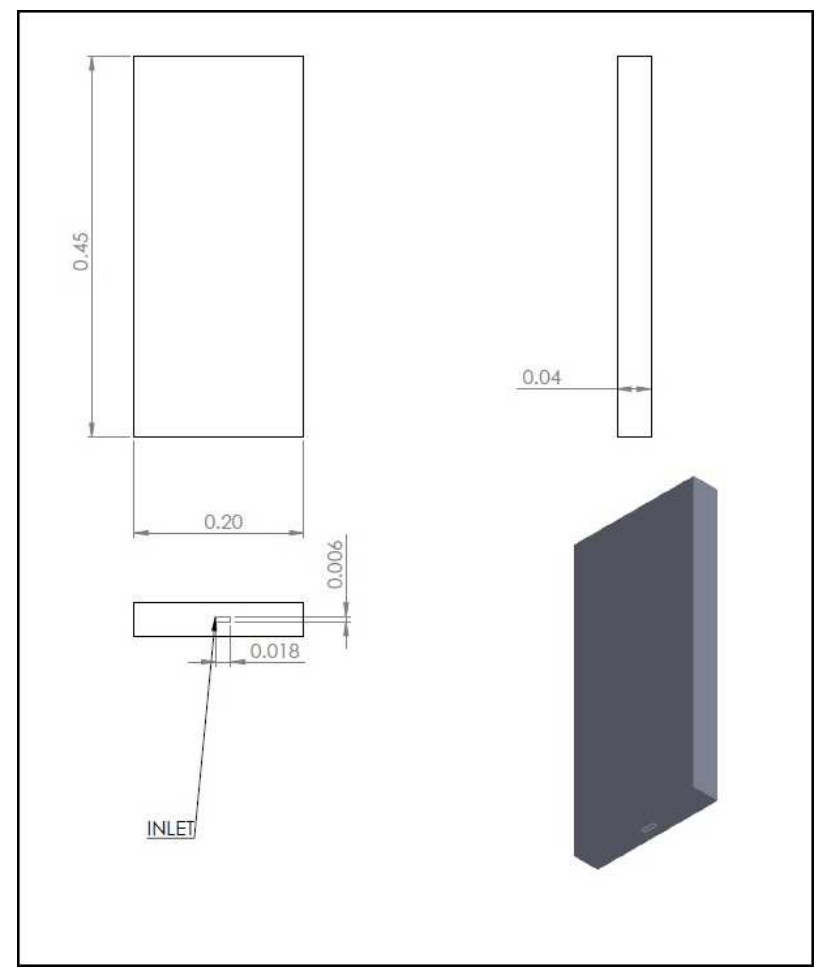

(a)

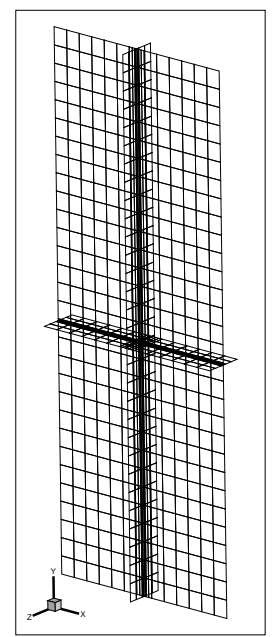

(b) $30 \times 17 \times 7$

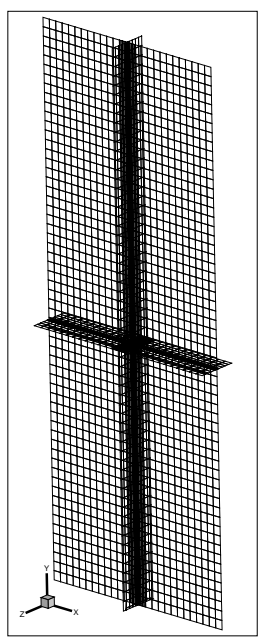

(c) $60 \times 34 \times 14$

Figure 4.1: (a)Dimensions of the setup used by (Diaz et al. , 2008) (b) the coarse grid (c) the refined mesh 
Table 4.4: Comparision of calculated frequencies with (Diaz et al. , 2008)

\begin{tabular}{|c|c|c|}
\hline$U_{G S}(\mathbf{m} / \mathbf{s e c})$ & Calculated Frequency (Diaz et al. , 2008) & Calculated Frequency \\
\hline 0.0024 & 0.0956 & 0.1099 \\
\hline 0.0071 & 0.1373 & 0.1709 \\
\hline 0.0120 & 0.2343 & 0.1892 \\
\hline 0.0166 & 0.3136 & 0.2200 \\
\hline 0.0213 & 0.3525 & 0.2497 \\
\hline
\end{tabular}




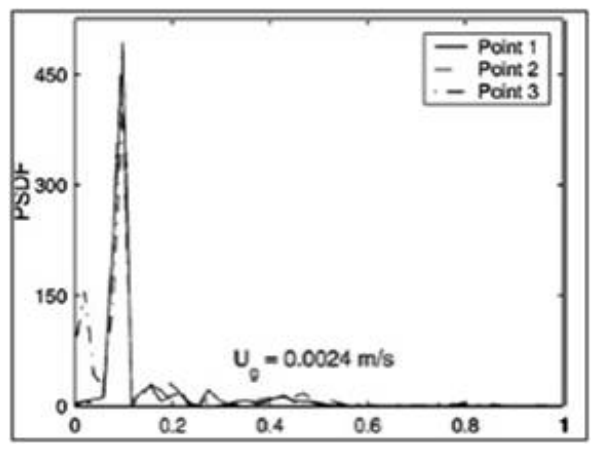

(a) $U_{g}=0.0024 m / s($ Diaz et al. , 2008)

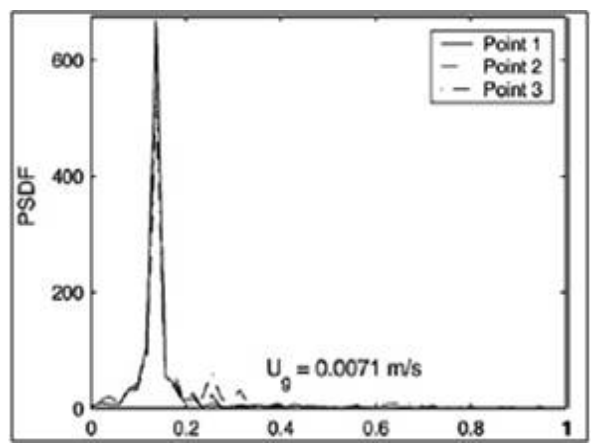

(c) $U_{g}=0.0071 \mathrm{~m} / \mathrm{s}($ Diaz et al. , 2008)

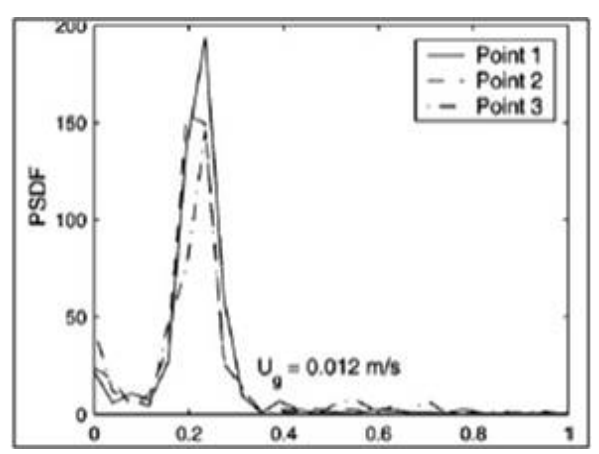

(e) $U_{g}=0.012 \mathrm{~m} / \mathrm{s}$ (Diaz et al. , 2008)

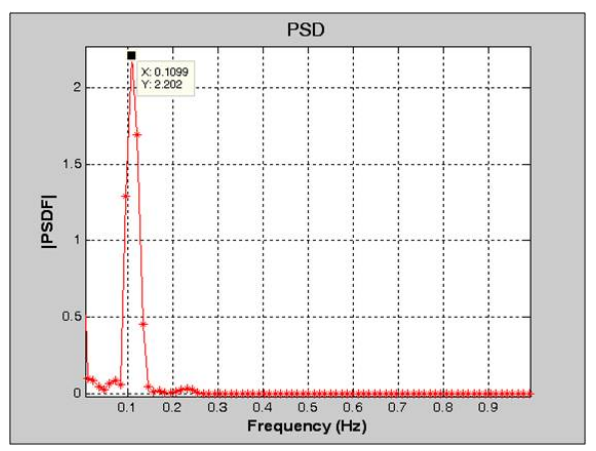

(b) $U_{g}=0.0024 \mathrm{~m} / \mathrm{s}$ Ansys-Fluent

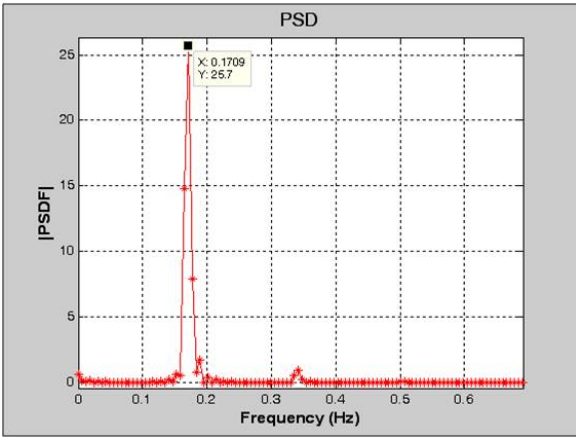

(d) $U_{g}=0.0071 \mathrm{~m} / \mathrm{s}$ Ansys-Fluent

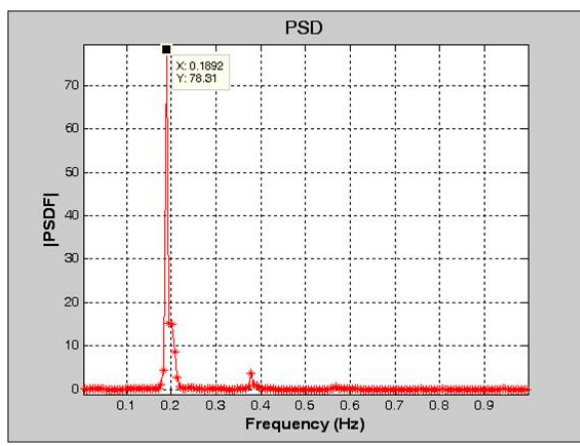

(f) $U_{g}=0.012 \mathrm{~m} / \mathrm{s}$ Ansys-Fluent

Figure 4.2: Calculated liquid velocity and the corresponding power spectral density function at different flow rates compared with results of (Diaz et al. , 2008), reproduced with permission. 


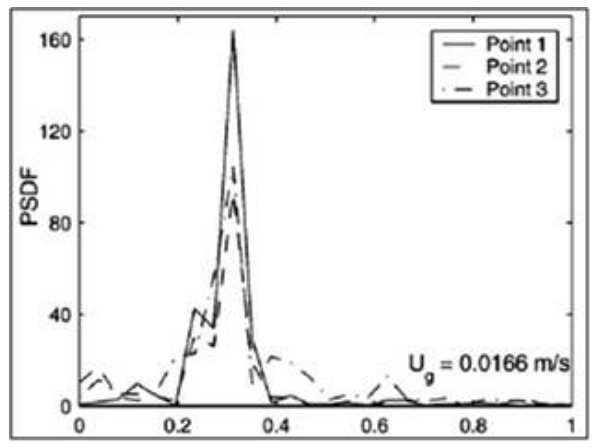

(g) $U_{g}=0.0166 \mathrm{~m} / \mathrm{s}$ (Diaz et al. , 2008)

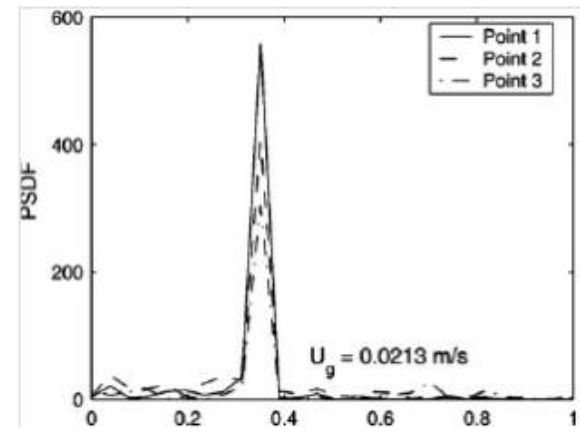

(i) $U_{g}=0.0213 \mathrm{~m} / \mathrm{s}$ (Diaz et al. , 2008)

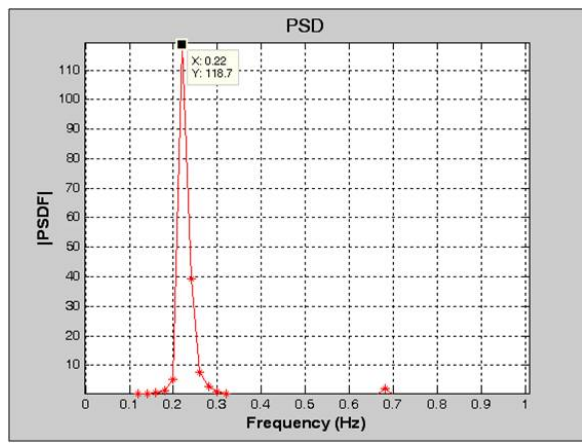

(h) $U_{g}=0.0166 \mathrm{~m} / \mathrm{s}$ Ansys-Fluent

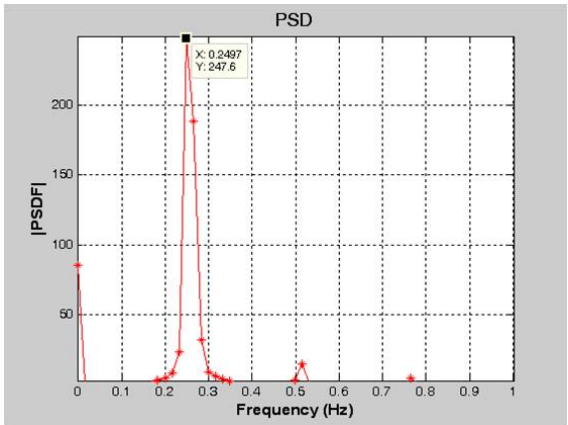

(j) $U_{g}=0.0213 \mathrm{~m} / \mathrm{sAnsys-Fluent}$

Figure 4.2: (Concluded) Calculated liquid velocity and the corresponding power spectral density function at different flow rates compared with results of (Diaz et al. , 2008), reproduced with permission. 


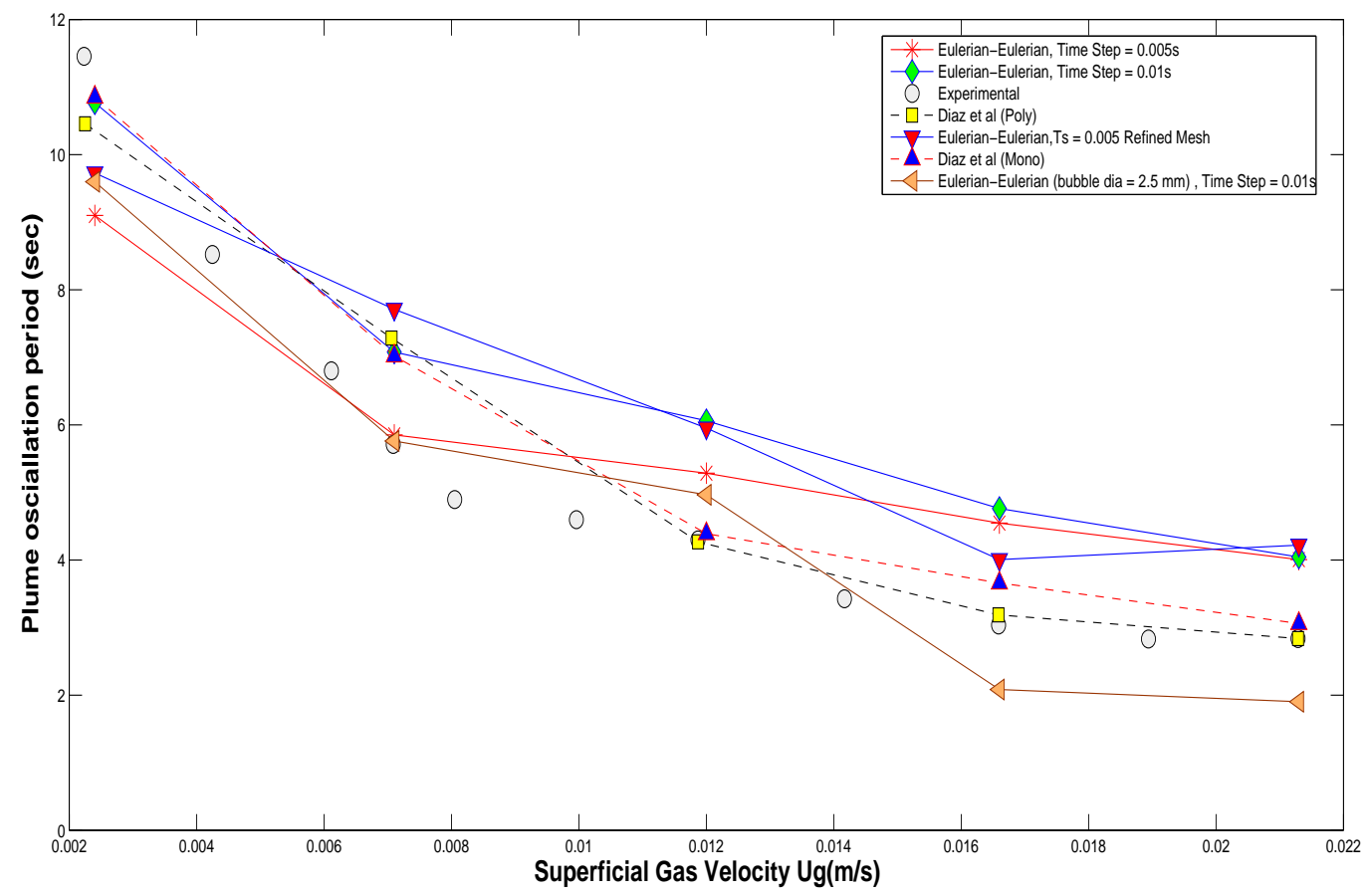

Figure 4.3: Comparison between calculated and experimental plume oscillation period, for E-E simulations and experiments by (Diaz et al. , 2008) 
The Fig. 4.3, consolidates all the results. To begin with, it is instructive to note that, all the attempts made in this present study display a reasonable agreement with the experimental values, barring some slight deviations. It is seen that for the coarse discretization level, the results get more agreeable with a drop in time step from $.01 \mathrm{sec}$ to $.005 \mathrm{sec}$, and the deviation between the two results wanes off at higher values of $U_{G S}$. The realization of another thought experiment, where the diameter of the bubble was dropped to $2.5 \mathrm{~mm}$ from $5 \mathrm{~mm}$, at a time step of $0.01 \mathrm{sec}$, showed a drop in plume oscillation period across the spectrum of inlet velocities studied. it also meant that this drop, showed a favourable change in the high velocity regions; whereas in the lower velocities the change in oscillation period is not favourable. Since, the bubble population is largely dominated by the smaller bubbles at high velocities the reduction of bubble diameter shows a favourible shift in the plume oscillation period at higher velocities.

It is also noted that, with refinement of grid the results remain about the same. The basic deviations from the experiments, could be attributed to the absence of a bubble coalescence and/or breakup model. The Fig. 4.4 shows a qualitative comparison between the experimental and numerical results obtained by (Diaz et al. , 2008), through the contour plots of the volume fractions of air. The prediction of the entrainment on either side of the plume, differs from (Diaz et al. , 2008), this is most probably due to the mono dispersed nature of the present work. Inclusion of a poly dispersed flow, accommodates the presence of bubbles smaller than what is used here $(5.5 \mathrm{~mm})$, thus encouraging the better prediction of the entrainment.

All the attempts tend to show a better agreement with the experiment at lower gas velocities, and are shown to be deviating at higher velocities. This observation is of particular significance, as the population of the smaller bubbles play a major role at higher velocities. Imposing a diameter of $5.5 \mathrm{~mm}$ is a considerable departure from reality, and is reflected in the results. Certain higher velocities, were revisited with smaller bubble diameter and they showed a values of POP lower and hence closer to the experiments. This phenomena is of particular interest in this study, and is shown to recurr in the following sections where the same problem is dealt with the E-L approach. 
Finally, the usage of the drag law as prescribed by (Grace et al. , 1976) proved crucial in attaining these results. Other attempts that were made included the drag correlation developed by (Schiller \& Naumann, 1935), which resulted in large deviations in calculated periodicity or sometimes the complete lack thereof. 


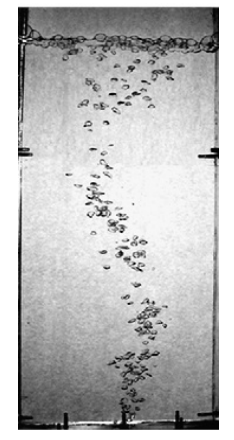

(a)

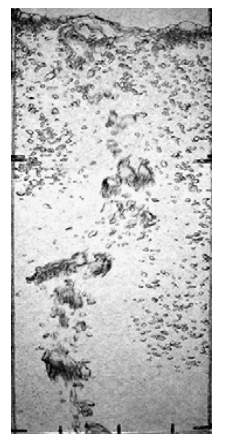

(d)

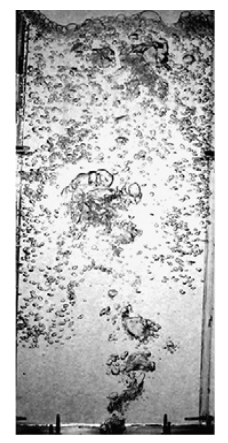

(g)

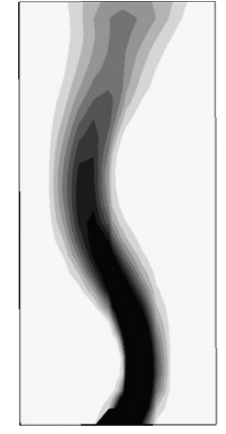

(b)

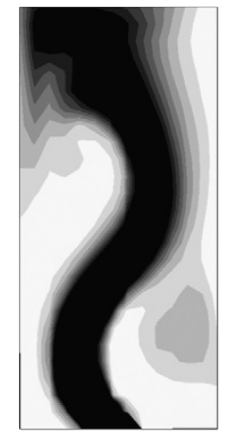

(e)

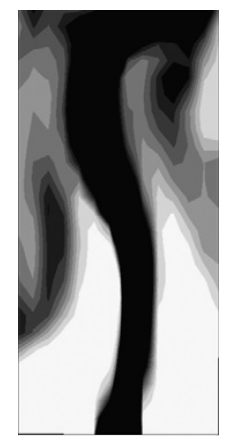

(h)

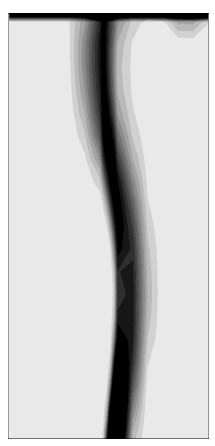

(c)

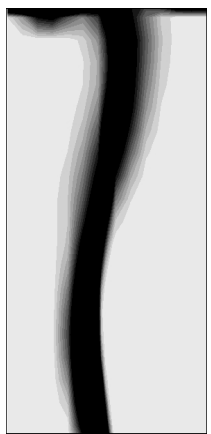

(f)

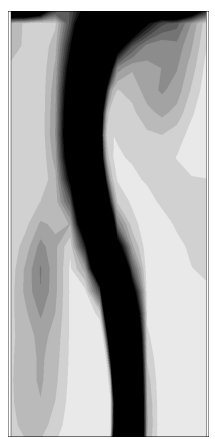

(i)

Figure 4.4: Contours of volume fraction of air in water at different $U_{g}$. (a,d,g) experimental (Diaz et al. , 2008);(b,e,h) computational (Diaz et al. , 2008); (c,f,i) computational present work 


\subsection{Eulerian-Lagrangian Simulations}

The set of simulations were performed using an inhouse LES based Navier Stokes solver with Lagrangian particle tracking (Hu \& Celik, 2008). The spatial resolutions for the E-L simulations were $36(\mathrm{w}) \times 16(\mathrm{~d}) \times \mathrm{x}$ (h) [Fig. ??], with an expansion ratio of 1.12 in the span-wise direction. The time step used was .005 and .001, with bubble diameters of $2.5 \mathrm{~mm}$ and $2 \mathrm{~mm}$. The construction of the Eulerian grid is independent of the particle size, as long as the particle size does not exceed an upper limit ( $\mathrm{Hu} \&$ Celik, 2008). This dependency of the grid size on the particle size needs further study. ( $\mathrm{Hu}, 2005)$.

The calculation of the plume oscillation in this case is similar to the one seen in

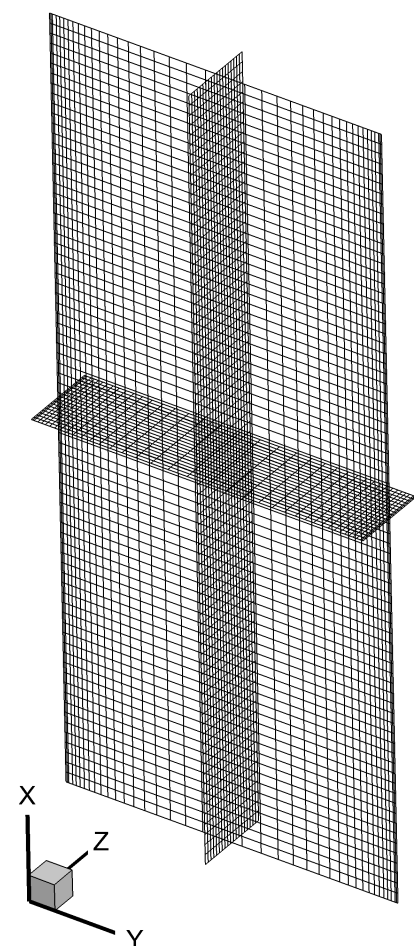

Figure 4.5: Grid used in the Eulerian-Lagrangian simulations

the E-E simulations. The velocities were again measured at a point offset from the centre, and then a Fourier transform performed to determine the dominant frequency. 
As mentioned earlier the primary difference with this approach is the treatment of the discrete bubble phase, in this case the air bubbles are treated as particles and not as a continuous media. Hence, the flow rate is controlled not just by injection velocity but also through the number of particles injected in a unit time. Thus there is a distinct difference in the definition of the inlet boundary condition, when compared with the E-E approach. It is also, essential that the air-bubbles that are assumed to be particles in their own right still do not coalesce or breakup, and more interestingly have a point volume. In other words, the particles themselves have no physical volume; and the volume fractions computed are through considerations for the particle diameter. The other far reaching consequence of this approach is that, because of the point volume nature of the particle, it does not result in the displacement of the liquid itself; hence no rise in liquid level is noticed with an increase in aeration.

As seen in Fig. 4.6, the POP values calculated through this approach, continue to be reasonably agreeable with the experimental values. The reduction of bubble diameter, shows a better agreement with the experiments for the mid value of the $U_{G S}$ spectrum, accompanied by a relative increase in the POP value for the lower values of $U_{G S}$ (away from experiments). For the higher values of $U_{G S}$ the results with the reduced $(2 \mathrm{~mm})$ diameter, show a favourable shift (towards the experiments) in the plume oscillation period. This "favorable" phenomena is very similar to what was observed in the E-E simulations as well. It could be considered to be indicative of some kind of a regime change, because of which at higher superficial velocities of gas, smaller bubbles tend to increase in the population. This is discussed at length in Section.4.3.1. However, to test the hypothesis in the numerical realm the data point at $U_{G S}=0.12 \mathrm{~m} / \mathrm{s}$ for the $2 \mathrm{~mm}$ diameter case has been taken as a reference, and a set of scaled diameters are arrived at through.

$$
d_{U_{G S}}=d_{r e f} *\left[\frac{U_{r e f}}{U_{G S}}\right]^{\frac{1}{2}}
$$

The exponential factor of $1 / 2$ is just perfunctory, for the time being. Such scaling gives a new set of diameters for a given velocity, as shown in Table. 4.5. The only exception is made in the case of the lowest velocity, where the prescribed diameter was $4.47 \mathrm{~mm}$, but because of the inability of the existing grid to accommodate larger particles, a diameter of $3.00 \mathrm{~mm}$ was chosen, the primary motivation being to keep the grid constant; which would ensure that the results are seen as 
solely effected by the change in diameter alone, and not due to the change in the discretization level. The results thus obtained shown in Fig.4.6 are closer to the

Table 4.5: Scaled Diameter for E-L Simulation

\begin{tabular}{|c|c|c|}
\hline$U_{G S}$ & Scaled dia $(\mathrm{mm})$ & Dia used $(\mathrm{mm})$ \\
\hline 0.0024 & 4.47 & 3.00 \\
0.0071 & 2.60 & 2.60 \\
0.0166 & 1.70 & 1.70 \\
0.0213 & 1.50 & 1.50 \\
\hline
\end{tabular}

experimental values, when compared to the base case of $2 \mathrm{~mm}$ diameter. This in a way, suggests that larger diameters play a role of prominence in the lower velocities, and as velocity increases the bubbles of smaller diameter rise to prominence.

Another factor that could be causing the variation is that the flow rate of air in these simulations is controlled through the number particles entering through the inlet in a unit time, as opposed to the inlet velocity itself. Therefore for a given velocity, a larger diameter would imply lesser number of bubbles entering the system.

An important difference when compared to the E-E method, apart from the treatment of the discrete phase, is that E-L case is LES based, and since the resolution of fluctuating quantities is more in the case of LES, it could effect the characteristic period of the system. 


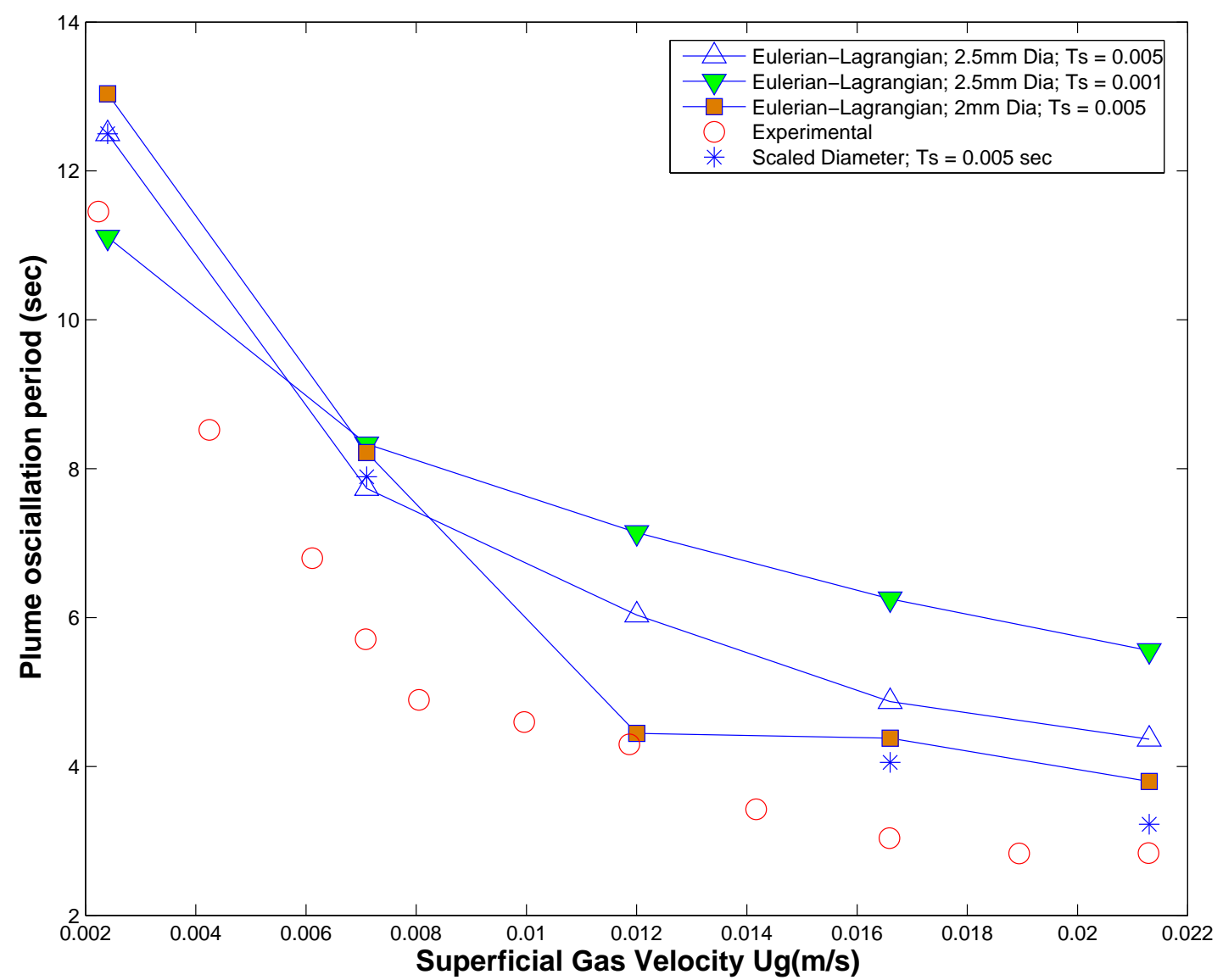

Figure 4.6: Comparison between calculated and experimental plume oscillation period, for E-L simulations and experiments by (Diaz et al. , 2008) 


\section{Simulation of a Viscous Case}

With the objective of better understanding the applicability of the EulerianLagrangian method in the case of higher viscosities, a case with the viscosity increased to 50 times that of water was pursued. In this attempt only the viscosity of the liquid was changed, and the diameter of the bubbles entering was fixed at $2 \mathrm{~mm}$. The grid used was 74x36x16 as shown in Fig.4.7 The increase in viscosity of the liquid meant higher number of particles got entrained, this could be largely due to the increase in viscous forces. Which translates to higher amount work needed to be done by the bubbles against the viscous forces due to the primary fluid, to break to the surface. The analysis of the velocity signals showed no considerable change in the dominant frequency with the velocity. These results are to be considered preliminary only, and the main motivation here is to investigate how the current E-L approach would handle an increase in viscosity. The main deviation that needs to be addressed yet, is the accommodation of the evolution of bubbles of larger diameter that come with liquids of higher viscosity, in a Lagrangian grid that is not too coarse for the Eulerian field and is LES worthy. Such an investigation at this stage, is left for further study.

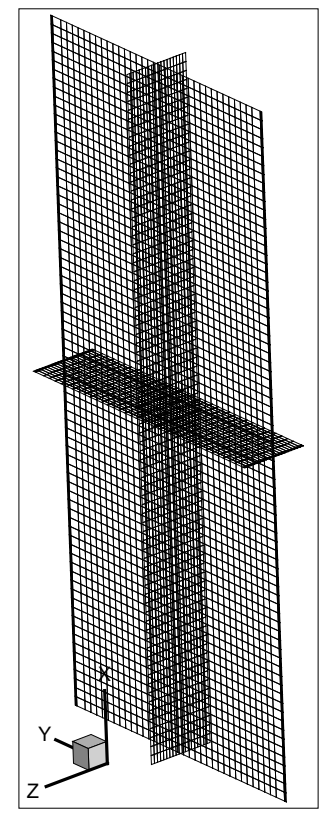

Figure 4.7: The Uniform Grid used for the simulation of a viscous case. $74 \times 36 \times 16$ 


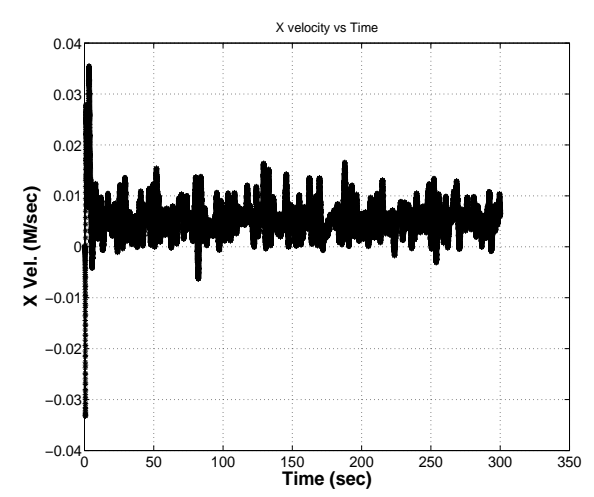

(a) $U_{G S}=0.0024$

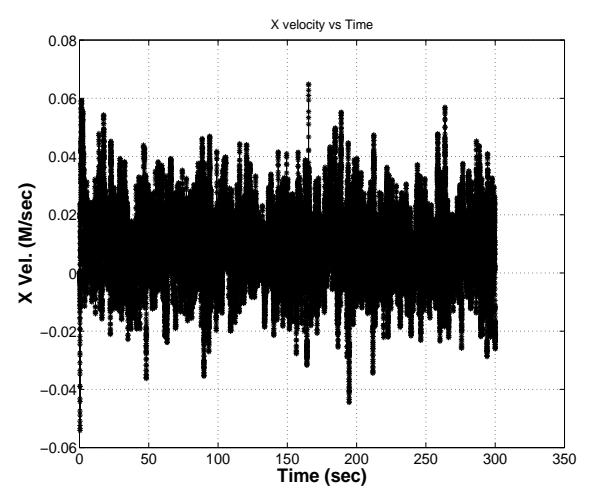

(c) $U_{G S}=0.0071$

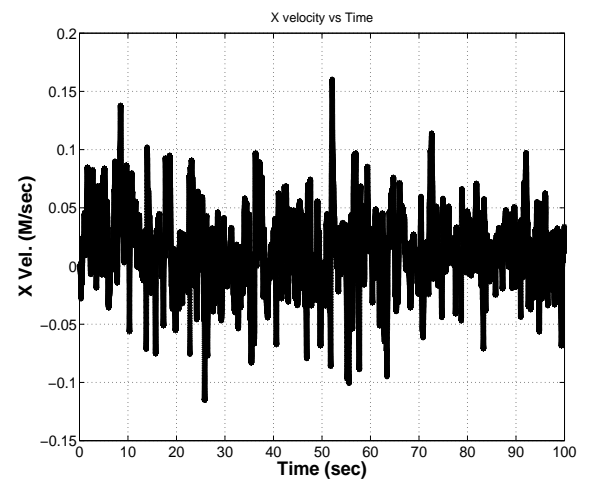

(e) $U_{G S}=0.012$

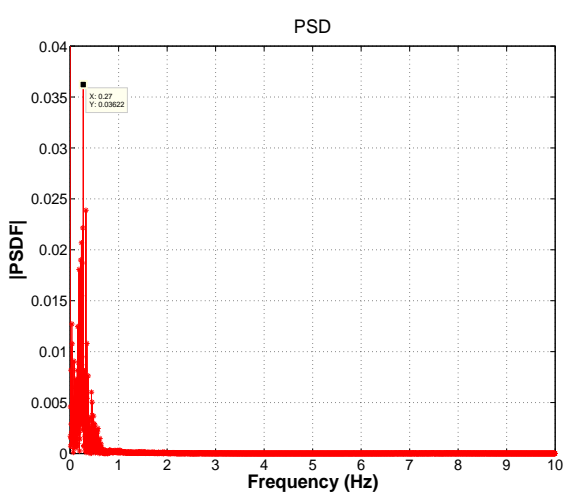

(b) $U_{G S}=0.0024$

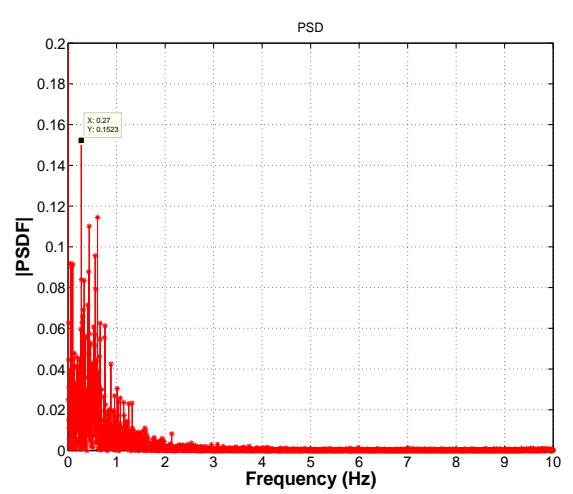

(d) $U_{G S}=0.0071$

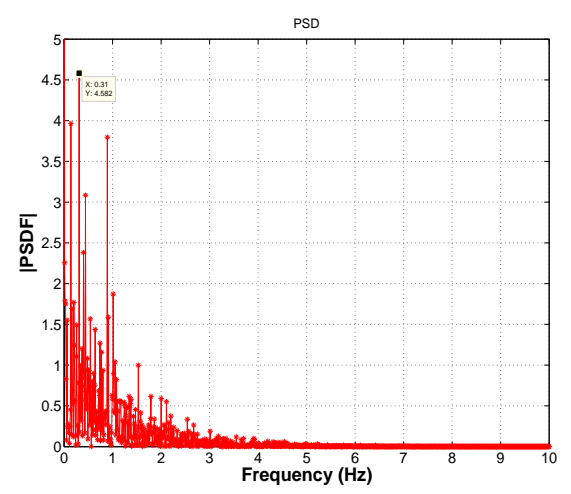

(f) $U_{G S}=0.012$

Figure 4.8: Calculation of liquid velocity and the corresponding power spectral density 
Shown in Fig. 4.8 is the set of velocity signals and their Fourier transforms. It is seen here that the peak power in power spectral density plot keeps rising with the velocity, although the dominant period is about the same. It is also seen that, many new set of semi-dominant frequencies are born at higher velocities. This 'mushrooming' of frequencies with increasing velocity, is most probably due to the higher entrainment of gas bubbles. With higher entrainment, the bubbles take longer to leave the system and hence at any given time the number of 'particle trajectories' was also higher than the plain water case. Therefore, with longer residence time the bubbles cause the overall gas hold up in the system to increase with velocity. This phenomena is well reflected in the Fig. 4.9; it also showcases that the code is sustainable; and that the number of particle tracks were infact attaining a steady state.

This study, uses an LES based solver, with a much finer mesh when compared with the attempts with the E-E method. It essentially also means that, since the effort with the E-E approach was through a RANS based turbulence model, the results thus obtained always concern themselves with the mean quantity rather than a true instantaneous value. In that respect, this LES based approach could be considered better at capturing the inherent turbulence charactersics. It can be recalled that LES while being the hope before DNS, resolves flow to a larger extent than RANS; therefore this fact could also have contributed to the sprouting up of the semi-dominant frequencies.

Gas hold up is a measure of the percentage (by volume) of gas present in the system. It is also essential to note, that with increase in velocity it was required to drop the time step for the numerical solution to be stable. For example for the lowest velocity a time step of $0.005 \mathrm{sec}$ was used and for $U_{G S}=0.0166 \mathrm{~m} / \mathrm{sec}$ a time step of $.001 \mathrm{sec}$. On some preliminary trials for $U_{G S}=.0213 \mathrm{~m} / \mathrm{sec}$ case a time step of $0.0005 \mathrm{sec}$ was required.

It is therefore seen that the E-L code does respond to an increase in viscosity; through prediction of higher gas holdups with increase in velocity. The emergence of a clearly dominant frequency does not happen, and instead a set of semi dominant frequencies are observed. The study of the true behaviour of a viscous fluid could be instructive at this stage to better understand the dynamics; therefore this 
forms the basis of the following section.

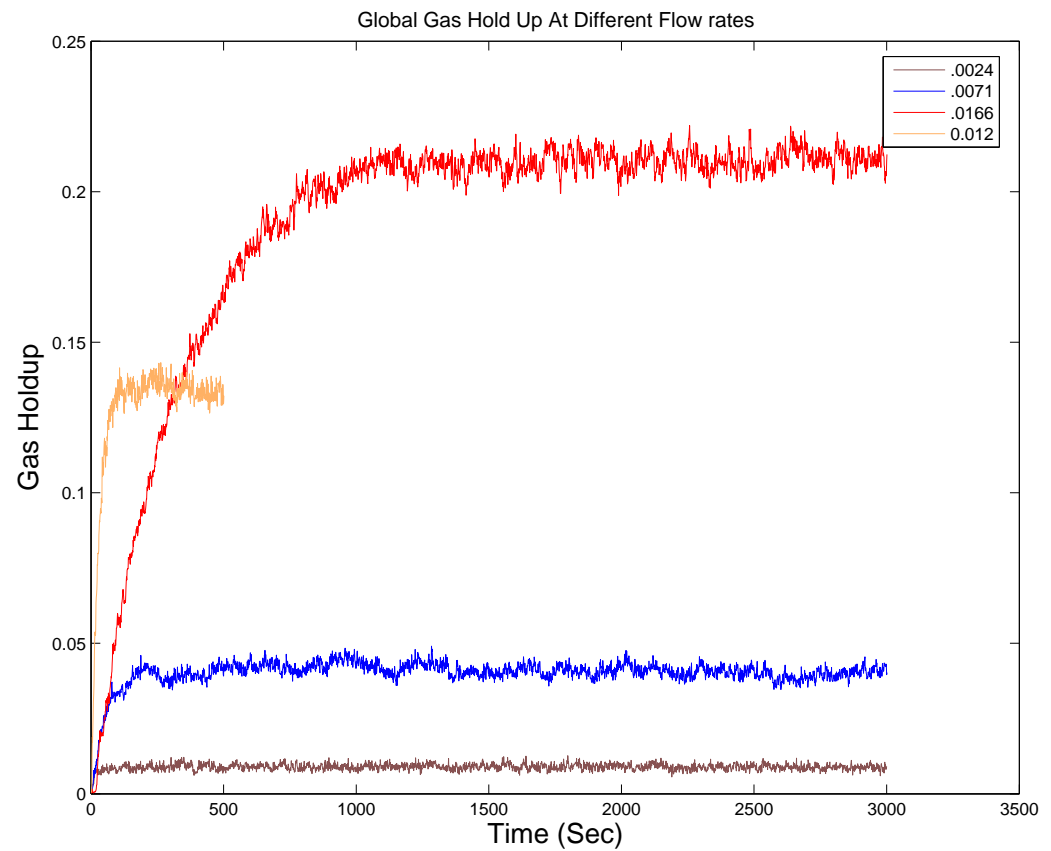

Figure 4.9: Gas Holdup vs Time 


\subsection{Experiments}

\subsubsection{Very Viscous Case}

For the hope of a better understanding the phenomena of two phase flow in very viscous media, certain experiments have been conducted in flat bubble columns as initial steps to quantify and to qualitatively depict the transition in bubbling regimes and thus by extension- mixing. These experiments are considered an extension to the work shown in Ravinuthala \& Celik (2013).

\section{The Setup}

The experimental setup is very similar to the one used by (Díaz et al. , 2008), in terms of the aspect ratio of the bubble column. For quantification two particular approaches have been resorted to; one is the measurement of pressure across the height of the column using a differential pressure transducer and the other is to make inferences about the bubble size distribution through image processing using high quality pictures of the bubble growth and coalescence in the column. The pressure transducer used is the OMEGA PX409-015DDUUSBH. The OMEGA USB Software, supplied by OMEGA has been used for the data acquisition.

The setup, consists of transparent glass for the front and back walls [Fig.??]. The stock air pump that the device came with, was dismounted, and replaced with a hose that was connected to a flow meter. That ensured control of the air flow rate through the column, which was critical in the study. The flow has been varied from $1 \mathrm{SCFH}$ (Standard Cubic Feet per Hour) to $20 \mathrm{SCFH}$. For each flow case the differential pressure fluctuations are sampled at a frequency of 320 Hz. In order to detect the presence of specific periodicity in the signal power spectral density analysis is performed, the results of which, as will be seen, could be very instructive in determining and quantifying to an extent the mixing present in the system. Also high resolution (12 MP) photographs have been taken of the resultant bubble plume, and then subjected to further image processing from the open source image software ImageJ (Rasband, 1997). Bubble size distribution thus obtained has a length scale of pixels. Histograms plotted from these data give an idea about the variation of the bubble size distribution with the flow rates. 


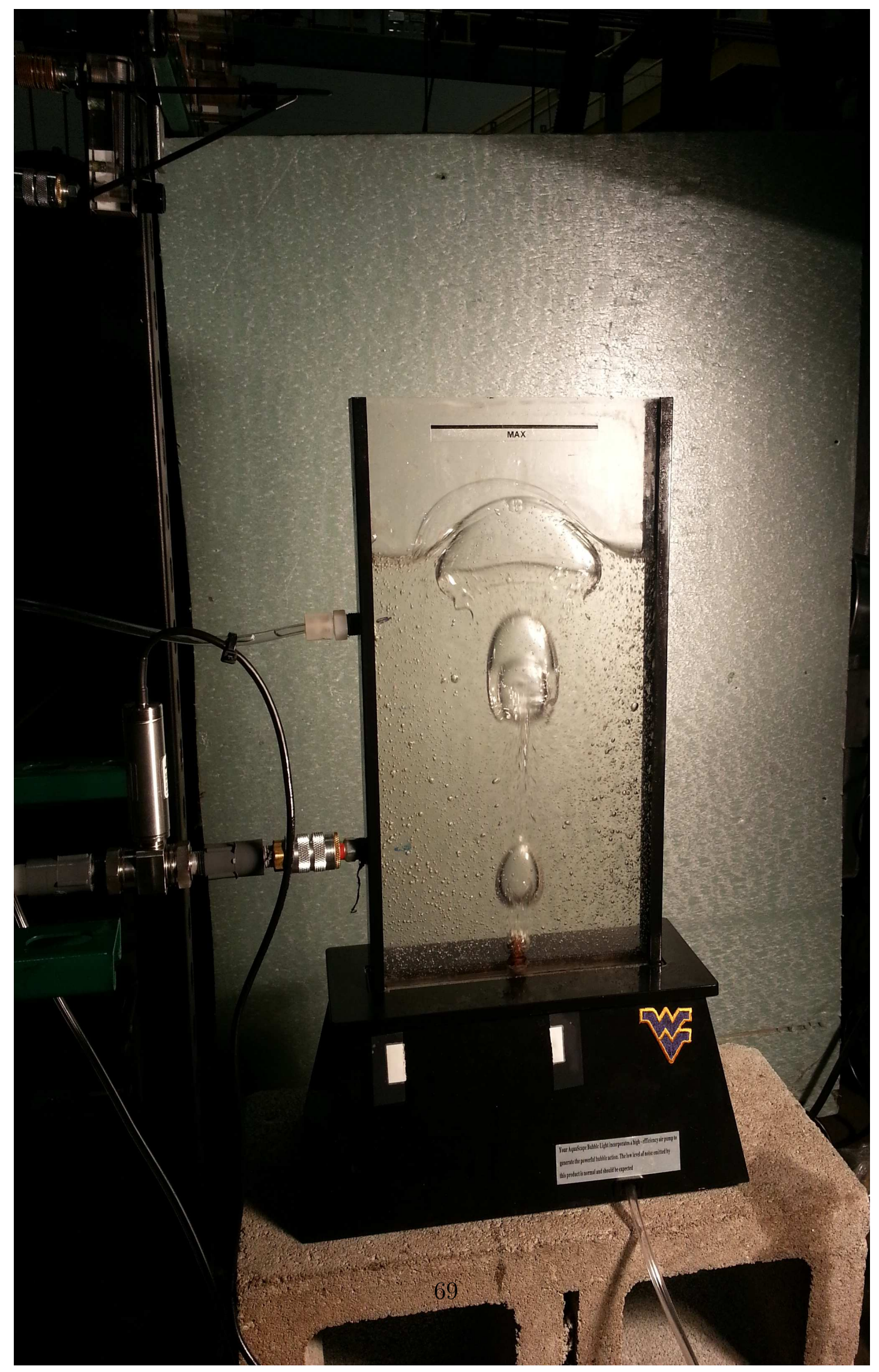

Figure 4.10: The experimental setup 


\section{The Pure Silicone Fluid}

Pure silicone fluid used for these experiments, has a viscosity of 350 CST. At this viscosity it is about 350 times as viscous as water. The physical properties of the fluids are given in Table. 4.6

Table 4.6: Pure Silicone Fluid (Polydimethylsiloxane) Data

\begin{tabular}{|c|c|c|}
\hline Property & Value & Units \\
\hline Specific Gravity & 0.970 & - \\
Refractive Index & 1.4032 & - \\
Pour Point & -50 & ${ }^{\circ} \mathrm{C}$ \\
Flash Point & 315 & ${ }^{\circ} \mathrm{C}$ \\
Surface Tension & 21.1 & dynes $/ \mathrm{cm}$ \\
Viscosity Temperature Coefficient & 0.60 & - \\
Specific Heat & 0.36 & BTU $/ \mathrm{lb.F}$ \\
\hline
\end{tabular}

\section{Bubble coalescence and break-up}

To understand the effects of bubble coalescence and breakup a few mean parameters need to be introduced.

The general mean diameter is expressed as:

$$
D_{p q}=\left[\frac{\sum_{i}^{t} n_{i} D_{i}^{p}}{\sum_{i}^{t} n_{i} D_{i}^{q}}\right]^{\left(\frac{1}{p-q}\right)}
$$

Under such a generic consideration of mean $D_{01}$ could be said to give an arithmetic mean. $D_{02}$ the area weighted mean and $D_{03}$ the volume weighted mean.

In general an arithmetic mean could be used to characterize a distribution, but due to the power law relationship between the diameter of the bubble and its volume and surface, a more complex formulation is desired.

The Global Sauter Mean Diameter which is defined as the diameter of a sphere that would have the same volume to surface area ratio as that of all the particles 
put together. It could then be expressed as

$$
D_{23}=\frac{\sum_{i}^{t} n_{i} D_{i}^{3}}{\sum_{i}^{t} n_{i} D_{i}^{2}}
$$

calculated on the basis in (Olmos et al. , 2001).

$$
d_{23}=\frac{1}{\sum_{i=1}^{t}\left(\frac{F_{i}}{d_{i}}\right)}
$$

where $F_{i}$ and $d_{i}$ are global mean size fraction and diameter of group $i$, respectively. (Díaz et al. , 2008) observed that in case of bubbly flows with water as the media; at values of $U_{G S}$ less than $2.4 \mathrm{~mm} / \mathrm{sec}$ the plume showed signs of developing vortical structures on either side, albeit without entraining the bubbles yet. The bubbles seemed clustered together near the inlet and spread out near the surface. This regime is termed as the dispersed bubble vortical flow

With further increase in $U_{G S}$ bubble clusters started to form, with the plume oscillating more often. Small bubbles now trapped and entrained by the vortices. This regime being termed as the transition vortical flow.

Finally at a higher values of $U_{G S}$ the phenomena of bubble coalescence and breakup starts becoming prominent, and as a result bubbles of different sizes begin to coexist. Large bubbles are observed in the centre while the smaller bubbles entrained in the vortices on the side descend along the wall. The downward motion of the liquid near the wall draws in the smaller bubbles nearer to the inlet, thus in a way ensuring complete aeration. The plume oscillation period was observed to be even quicker and the regime termed as fully developed vortical flow.

The study of the variation of the Sauter diameter with $U_{G S}$ is of significance. (Olmos et al. , 2001) , (Camarasa et al. , 1999) state that it enables in visualizing the onset of the aforementioned regimes. The increase of Sauter mean diameter could be seen as a measure of the coalescence phenomena taking precedence. Once the Sauter mean diameter reaches a peak values it then begins to decrease with increase in flow rate; this could be said to be the regime where breakup becomes prominent. Then with further increase in flow rate the Sauter diameter is not seen to change anymore indicating the equilibrium of the coalescence and breakup 
phenomena. Thus it is well established that, the variation of the mean diameter (in this case SMD) could be seen as a good representation of the influence of coalescence and breakup phenomena.

The question then arises about our specific interest in this. This keen interest is well backed by the proposition that, the vortical structures caused due to the interaction of these bubbles and the inherent unsteadiness of the column plays a favorable role in enhancing the mixing, which is a desired entity in the design requirements of any submerged combustion melter. In other words, the above mentioned phenomena that causes mixing should speed up all forms of transport phenomena(Buwa \& Ranade, 2004), which is desired in industry scale applications. What effect then increasing viscosity would have on the dynamics as observed by (Díaz et al. , 2008). That is the essence of the discussion presented in this section.

\section{Global Gas Holdup}

The global gas hold up is obtained through manometric methods as discussed in (Ruthiya et al. , 2005)

$$
\alpha_{g}=1-\frac{\left(\overline{\Delta P_{\text {aerated }}}\right)}{\left(\overline{\Delta P_{\text {non-aerated }}}\right)}
$$

where $\overline{\Delta P}$ represents the time averaged pressure drop sampled at a frequency of $320 \mathrm{~Hz}$ in the present work. If the probes of the differential pressure transducer are a distance $h$ apart, then

$$
\begin{gathered}
\Delta P_{\text {non-aerated }}=\rho_{\text {liquid }} \cdot g \cdot h \\
\Delta P_{\text {aerated }}=\rho_{\text {eff }} \cdot g \cdot h
\end{gathered}
$$

where

$$
\rho_{\text {eff }}=\alpha \rho_{\text {air }}+(1-\alpha) \rho_{\text {liquid }}
$$

where $\alpha$ is the volume fraction of air in the liquid. Eq. 4.5 now effectively becomes

$$
\alpha_{g}=1-\frac{\rho_{\text {eff }}}{\rho_{\text {liquid }}}
$$




\subsubsection{Results and Analysis}

\section{Analysis from Digital Imagery}

Fig. 4.11 shows a series of photographs taken, of the rising bubbles in the pure silicone fluid. The objective is to understand qualitatively, the hydrodynamics of the bubbles rising, and in a way to assess and hopefully quantify the amount of mixing obtained. It is essential to be reminded that any transport phenomena would be accelerated if a better mixing is obtained. Especially in the case of submerged combustion melting, its is desired that the hot gas blobs DO NOT escape as slugs, for even a single blob of hot gas breaking up at the surface and escaping into ambiance would definitely be looked upon as a wasteful event. As opposed to having the blob breakup and be entrained in the fluid in the form of a set of smaller bubbles thus ensuring the heat is not lost, and that maximum possible surface area is ensured for any desired chemical reaction to be facilitated/ accelerated.

The processed images ensure the study of just that, it is clearly seen through mere visuals that at higher velocities the number of smaller bubbles have increased significantly. The bubble size distribution is provided in the form of histograms Fig. 4.15. The units for the diameter are maintained in pixels, for the distribution obtained by centimeter as the unit is provided in Appendix B. It is essential to note that all the diameters were calculated based on the frontal projection area, hence could be considered as area equivalent diameters. An effort could be made in future, to obtain lateral images of the column which would provide the additional information needed to compute the volume equivalent diameter, thus having a better representation of the physics.

Another approach to better understand the phenomena is akin to the one used by (Olmos et al. , 2001) and (Camarasa et al. , 1999). It is to see the evolution of the mean diameter with increasing flow rate, which shall eventually throw light on the coalescence and breakup phenomena and which one of those is more predominant. (Olmos et al. , 2001) had used the Sauter mean diameter to conduct such a study. It is is also recalled that the fluid under consideration by them was water, much less viscous than the silicone fluid and with considerably different surface tension. What this eventually means is that, the number of bubbles entrained is 
higher for the silicone fluid when compared to water, accompanied by the fact that the singular big mushroom like bubbles are absent in the water. So the size difference between the larger and smallest bubble could be said to be higher in the case of the silicone fluid, with the number of the tiny bubbles also being higher. Under such a scenario where the population is dominated by the smaller bubbles, and the larger bubbles being the outliers, the Harmonic Mean $(\mathrm{H})$ could be considered a fairer approximation of the entire population. Based on the Eq. 4.2 the harmonic mean could be arrived through the formulation $D_{-10}$

$$
D_{-10}=\left[\frac{\sum_{i}^{t} n_{i} D_{i}^{-1}}{\sum_{i}^{t} n_{i} D_{i}^{0}}\right]^{-1}
$$

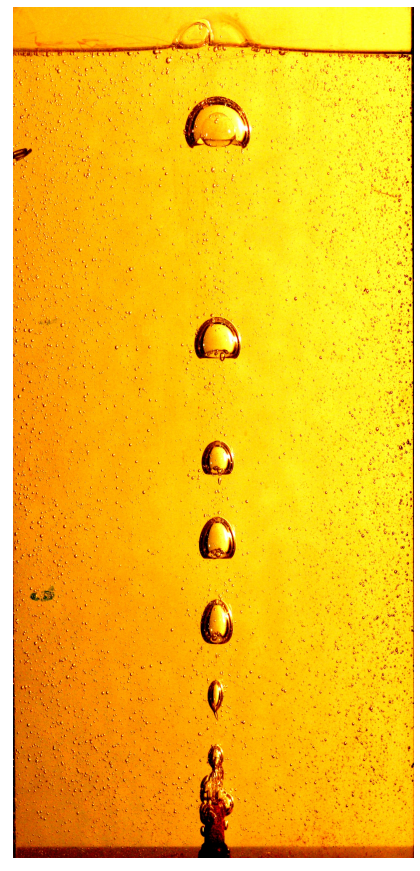

(a) $1 \mathrm{SCFH}$

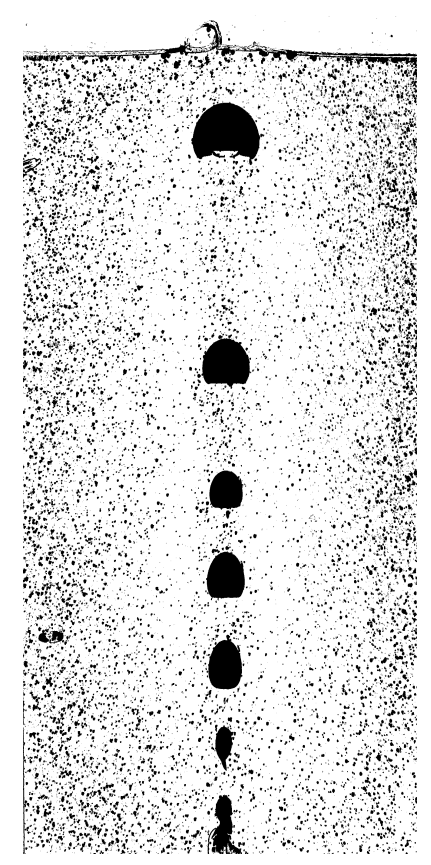

(b) 1SCFH Processed

Figure 4.11: Digital photographs of gas pockets in the viscous liquid media, The binary images obtained through edge detection and image processing from imageJ $[1 \mathrm{SCFH}]$

The Fig. 4.13 is derived from visual observation the histograms available in Fig. 4.15. The $\mathrm{x}$ axis in the histograms indicate the bubble size (diameter) in 


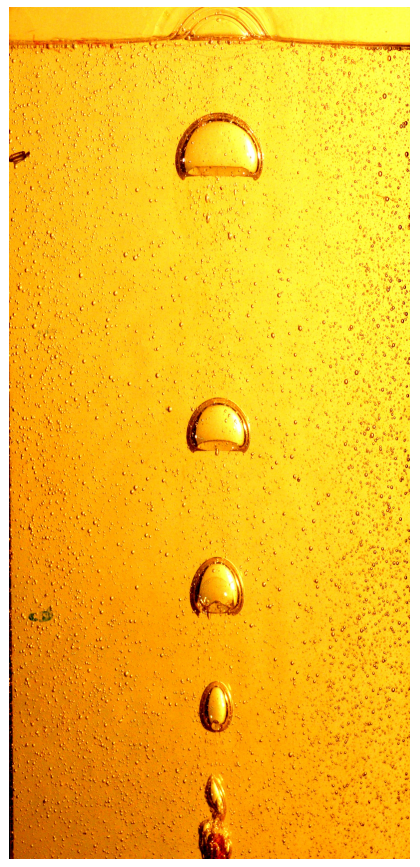

(c) $2 \mathrm{SCFH}$

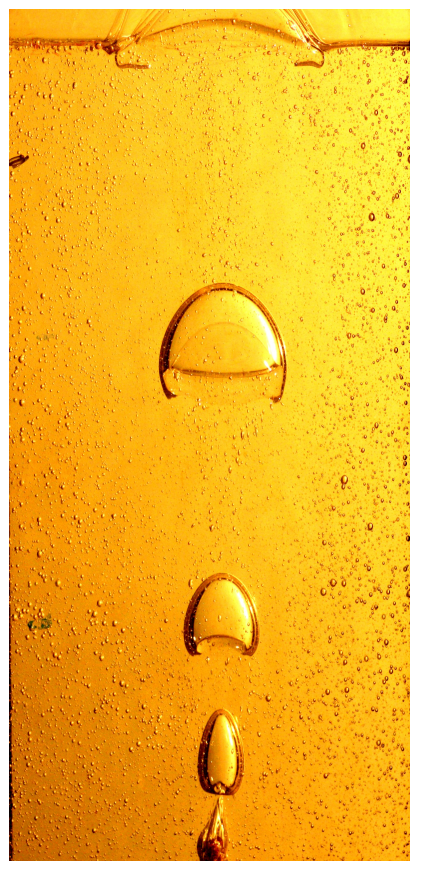

(e) $6 \mathrm{SCFH}$

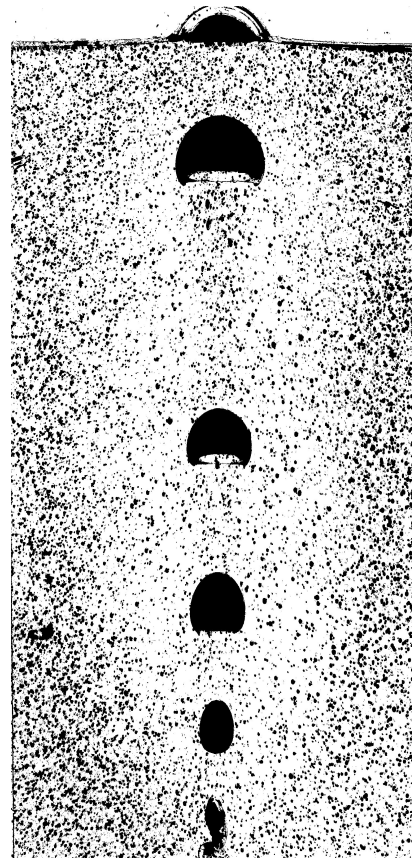

(d) $2 \mathrm{SCFH}$

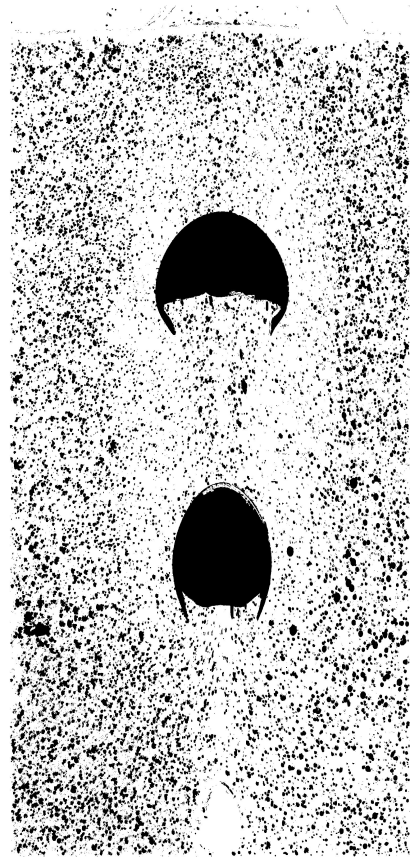

(f) $6 \mathrm{SCFH}$

Figure 4.11: (Continued) Digital photographs of gas pockets in the viscous liquid media, The binary images obtained through edge detection and image processing from imageJ $[2-6 \mathrm{SCFH}]$ 


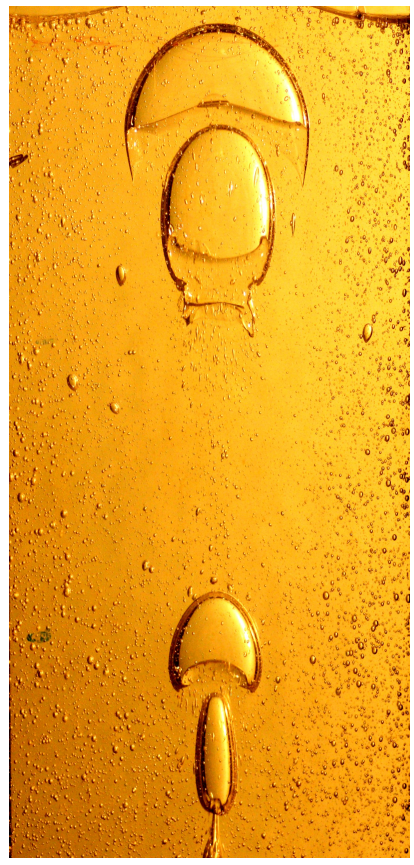

(g) $8 \mathrm{SCFH}$

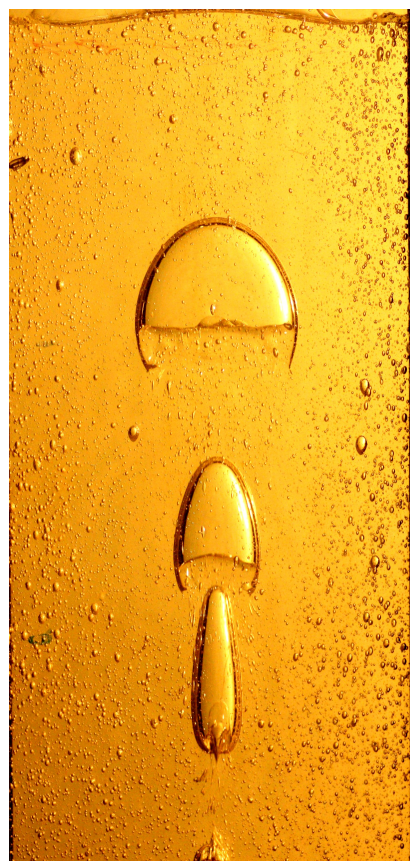

(i) $10 \mathrm{SCFH}$

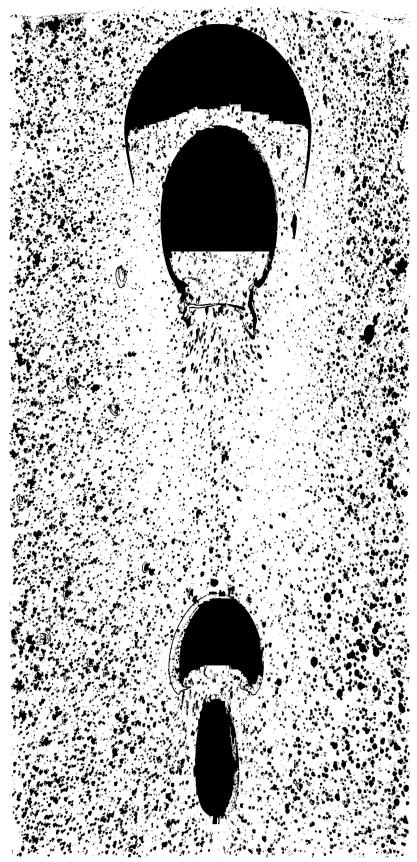

(h) $8 \mathrm{SCFH}$

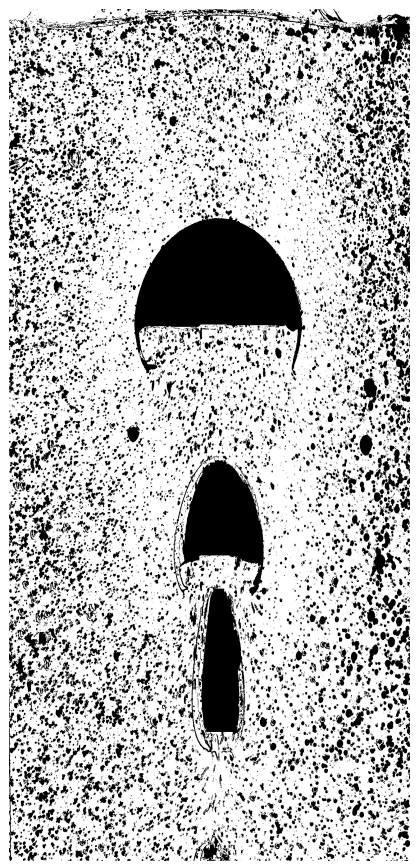

(j) $10 \mathrm{SCFH}$

Figure 4.11: (Continued) Digital photographs of gas pockets in the viscous liquid media, The binary images obtained through edge detection and image processing from imageJ [8-10 SCFH] 


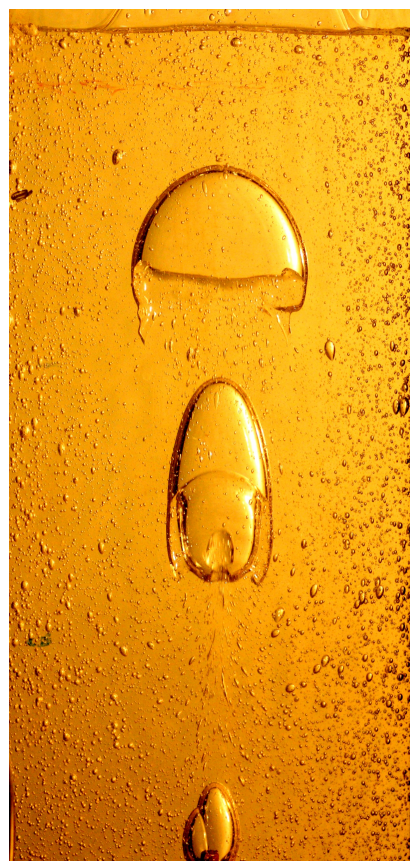

(k) $11 \mathrm{SCFH}$

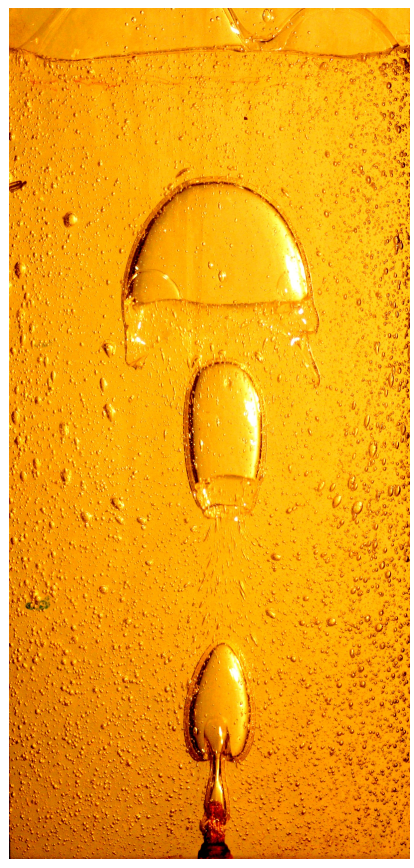

(m) $14 \mathrm{SCFH}$

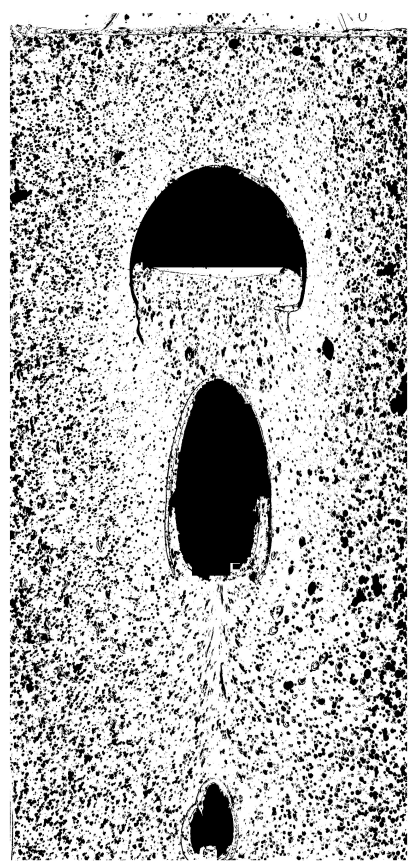

(l) $11 \mathrm{SCFH}$

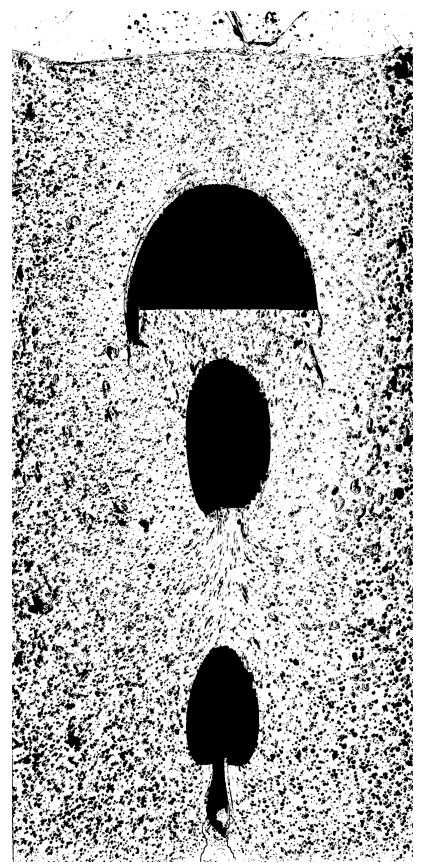

(n) $14 \mathrm{SCFH}$

Figure 4.11: (Continued) Digital photographs of gas pockets in the viscous liquid media, The binary images obtained through edge detection and image processing from imageJ [11-14 SCFH] 


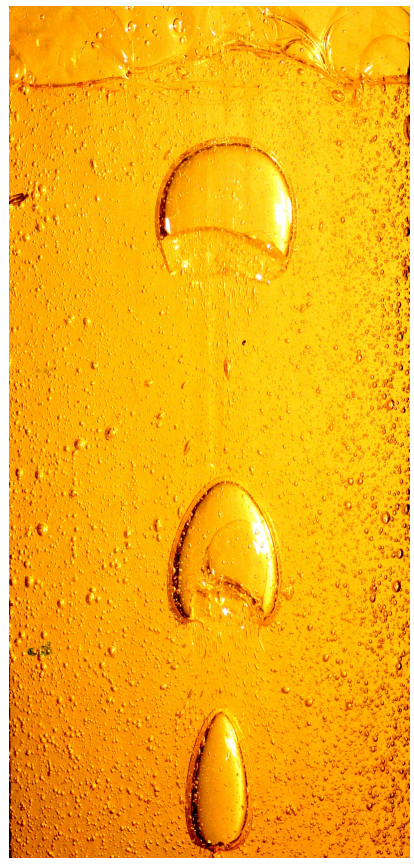

(o) $16 \mathrm{SCFH}$

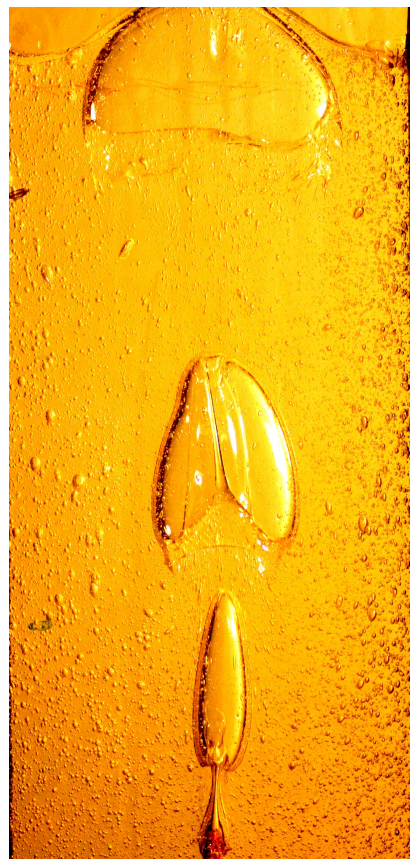

(q) $18 \mathrm{SCFH}$

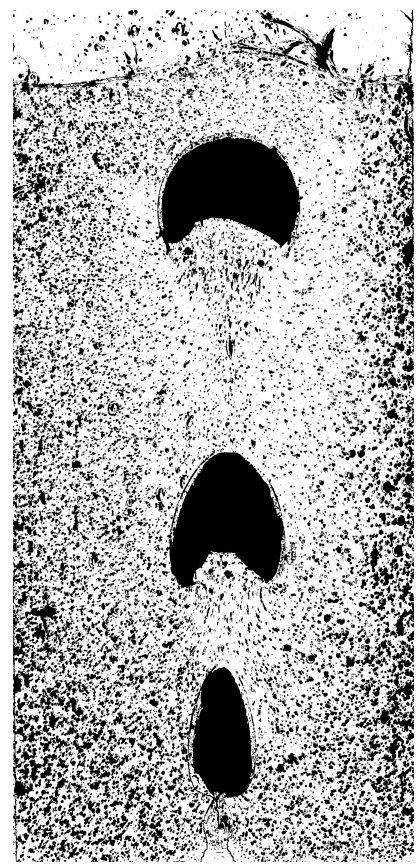

(p) $16 \mathrm{SCFH}$

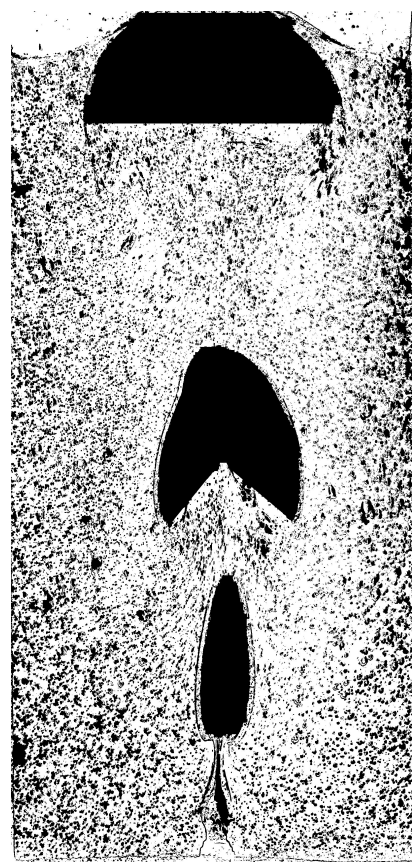

(r) $18 \mathrm{SCFH}$

Figure 4.11: (Concluded) Digital photographs of gas pockets in the viscous liquid media, The binary images obtained through edge detection and image processing from imageJ [16-18 SCFH] 


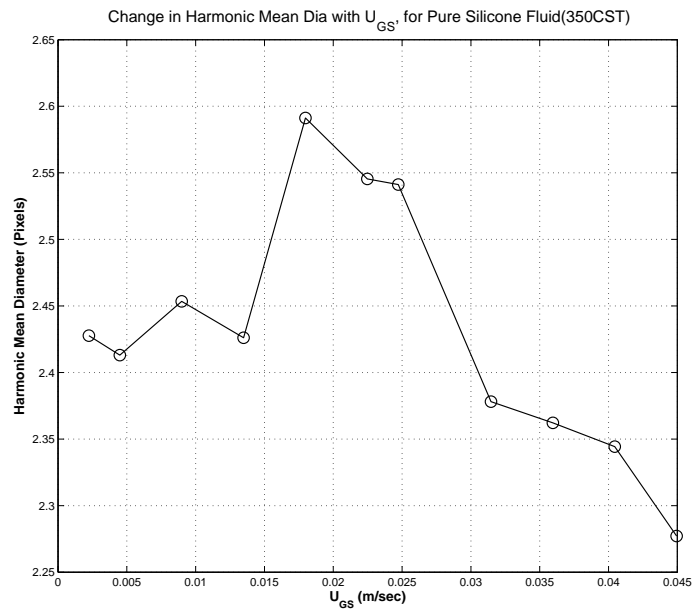

Figure 4.12: Variation of the Harmonic Mean Diameter

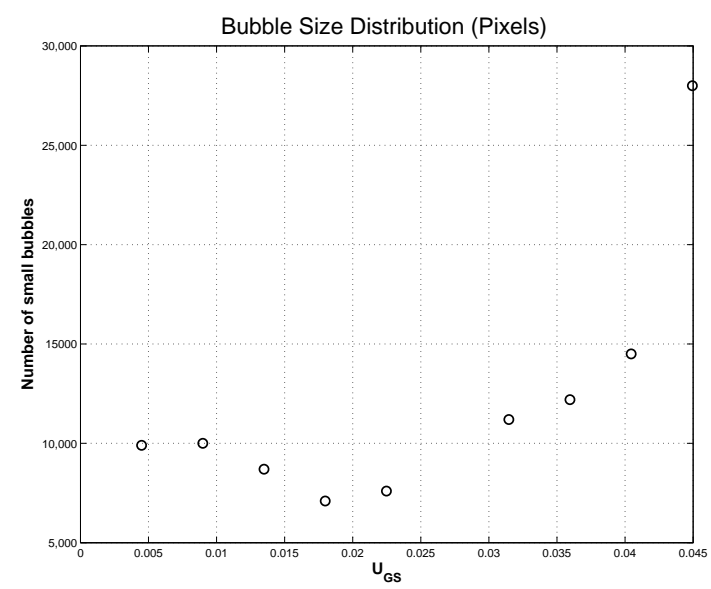

Figure 4.13: Variation of small bubble distribution 
pixels (for the disribution in cm pleaser refer Appendix B), and the peak value of the histogram reflects the population of the small bubbles. This is used as the basis for the Fig. 4.13; where, with an increase in the velocity, initially a drop in the population of the small bubbles is noticed. With further increase in the velocity, the population of the small bubbles embarks on a steep climb, showcasing that at higher values of superficial gas velocity the entrainment of smaller bubbles is significant and climbs with increase in velocity.

At this stage the proposition being advanced is that, the peak attained in Fig. 4.12 at a $U_{G S}$ value of $0.017 \mathrm{~m} / \mathrm{sec}$ is not circumstantial but is justified through the variation of the small bubble population [Fig. 4.13] which also sees a low at the same value of superficial gas velocity. It is also essential to take note of the fact that the variation of the Harmonic Mean Diameter, as seen in Fig. 4.12 appears exaggerated; i.e the variation observed is not by a significant amount as compared to what is seen in the case of the Sauter Mean Diameter Fig. 4.14.

It would then be a fair working hypothesis to state that the coalescence phenomena is dominant until the value of $0.017 \mathrm{~m} / \mathrm{sec}$, where as the breakup of bubbles / entrainment of the smaller bubbles increases for an increase in the superficial velocity after that. That is of course, accompanied by an increase in the population of the smaller bubbles. However, the variation of the Sauter Mean diameter [Fig. 4.14] does not correlate to the trend seen in the variation of the harmonic mean diameter, and that could be attributed to the presence of extreme outliers (large size gradient between the large bubbles and the smaller ones); making the harmonic mean a fairer approximation of the mean.

Table 4.7: Conversion of flow rate from $\mathrm{SCFH}$ to $U_{G S}$

\begin{tabular}{|c|c|c|c|c|c|c|c|c|c|c|}
\hline $\mathrm{SCFH}$ & 2 & 4 & 6 & 8 & 10 & 12 & 14 & 16 & 18 & 20 \\
\hline$U_{\text {GS }}(\mathrm{m} / \mathrm{sec})$ & 0.004 & 0.009 & 0.013 & 0.018 & 0.023 & 0.027 & 0.032 & 0.036 & 0.040 & 0.045 \\
\hline
\end{tabular}




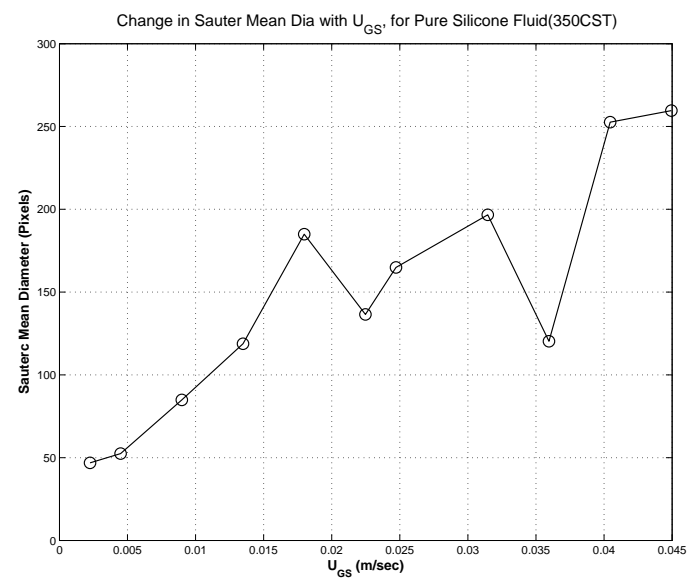

Figure 4.14: Variation of the Sauter Mean Diameter 


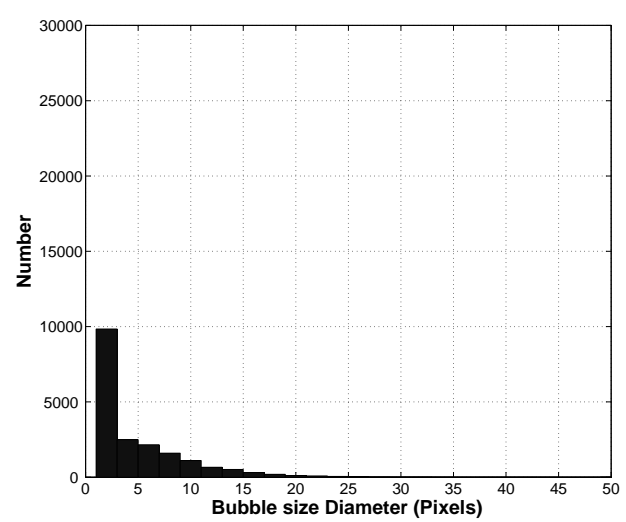

(a) $2 \mathrm{SCFH}$

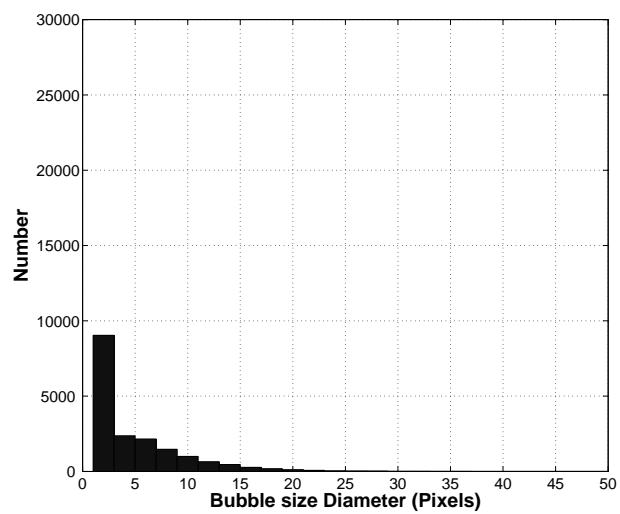

(c) $6 \mathrm{SCFH}$

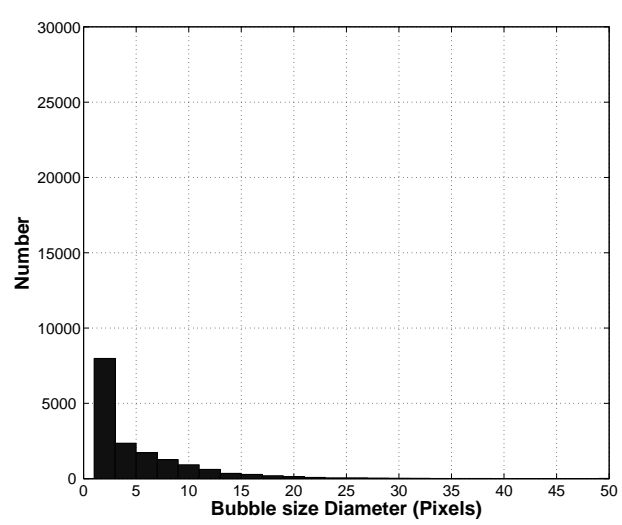

(e) $10 \mathrm{SCFH}$

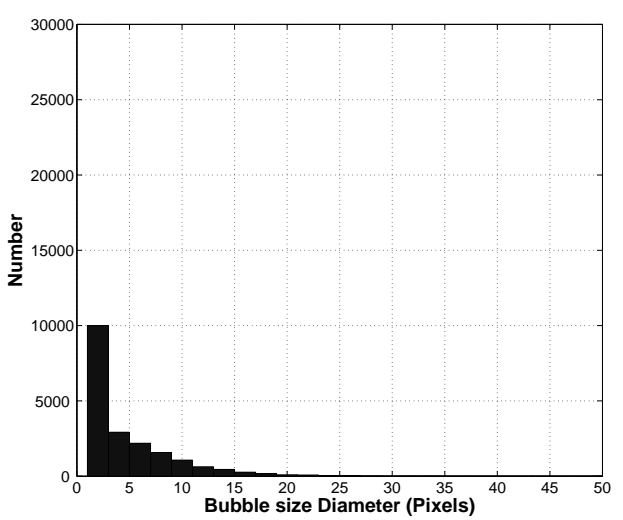

(b) $4 \mathrm{SCFH}$

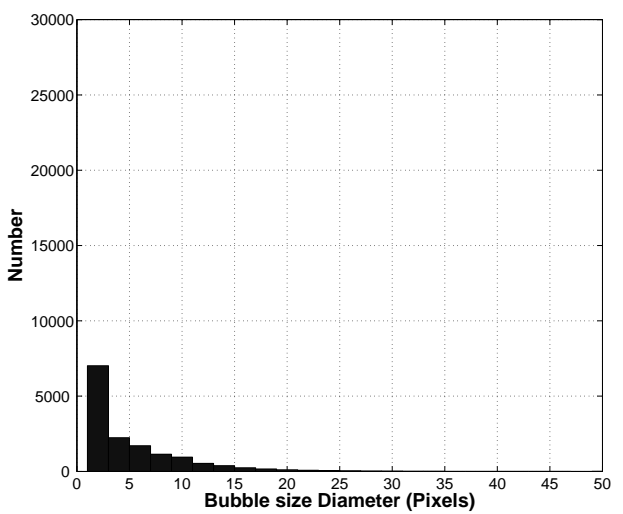

(d) $8 \mathrm{SCFH}$

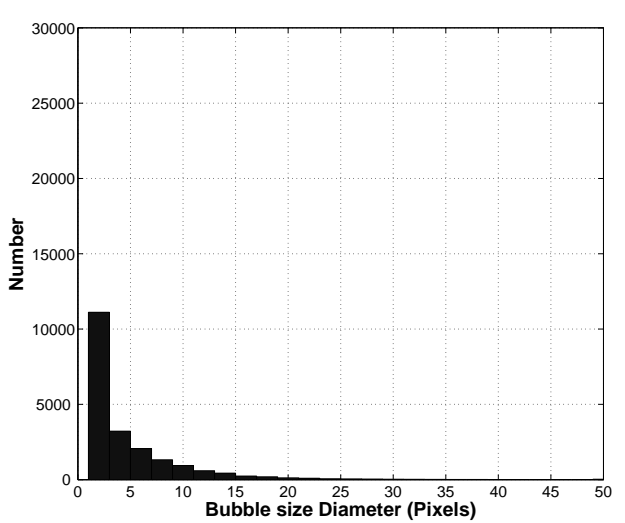

(f) $14 \mathrm{SCFH}$

Figure 4.15: Particle size (in pixels) distribution for[2-14 SCFH] from the image analysis using ImageJ. 


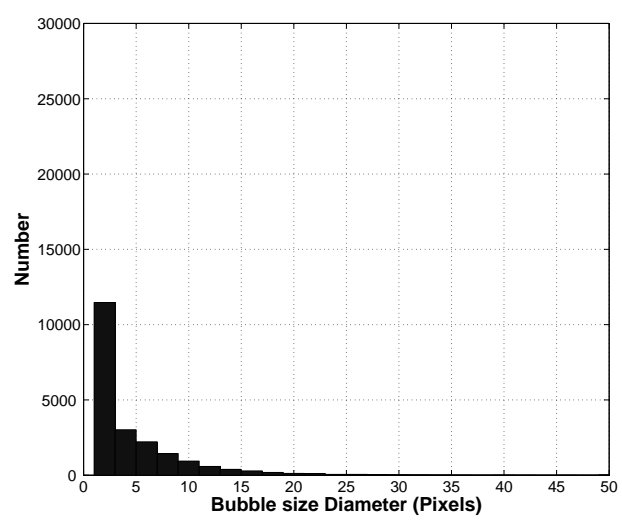

(g) $16 \mathrm{SCFH}$

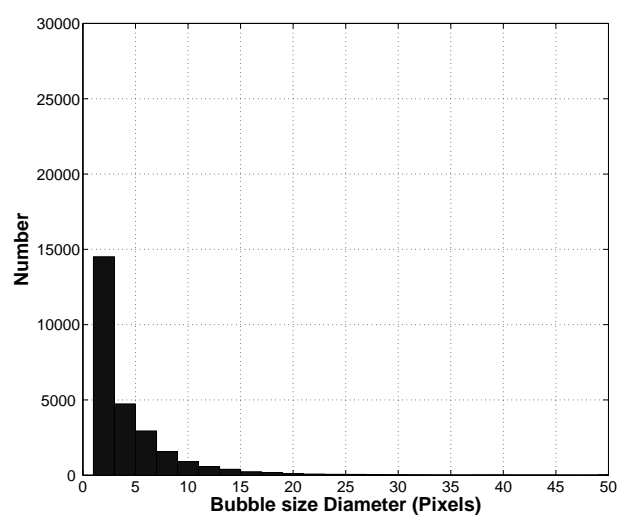

(h) $18 \mathrm{SCFH}$

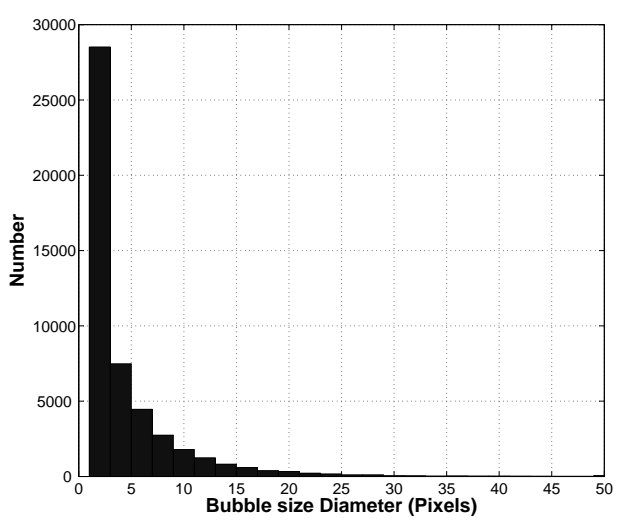

(i) $20 \mathrm{SCFH}$

Figure 4.15: Particle size (in pixels) distribution for[18-20 SCFH] from the image analysis using Image J. 
The Fig. 4.16 gives details of the pressure time series signal and its corresponding power spectral density. It is seen that with increase in velocity, the dominant frequency and power associated with it does not change considerably. But, it is interesting to notice a sudden spike in power from 0.01 at $6 \mathrm{SCFH}$ to 0.09 at 8 $\mathrm{SCFH}$; it was at $8 \mathrm{SCFH}$ that the peak in the harmonic mean diameter was noticed Fig. 4.12. Another observation, is the increase in the 'mushrooming' of the higher frequencies with velocity. This behaviour, is analogous to increased mixing that is prevalent at higher velocities. This inference is consistent with the observations made from the Fig. 4.11. Therefore, mixing could be characterized through the higher frequencies of low power, and their growth becomes distinctly prominent at higher velocities where the breakup phenomena takes over and results in an increase in the number of small bubbles.

Fig. 4.17 shows that the global gas holdup varies almost linearly with the superficial gas velocity. Which means more bubbles are entrained at higher velocities. This being a preliminary investigation, flow rates above 20 SCFH couldn't be explored because of design constraints. The time averaging of photographic data is also essential, for capturing the unsteadiness and to get a better estimate of bubble growth and therefore a better assessment of mixing. 


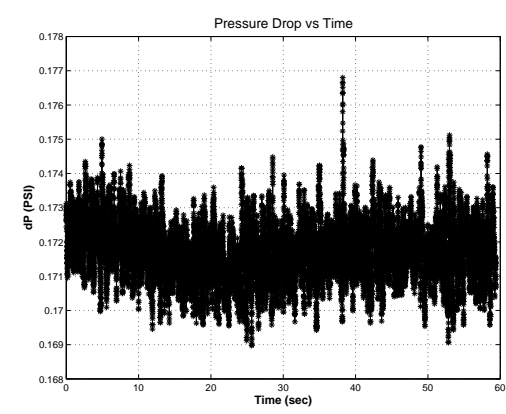

(a) $2 \mathrm{SCFH}$

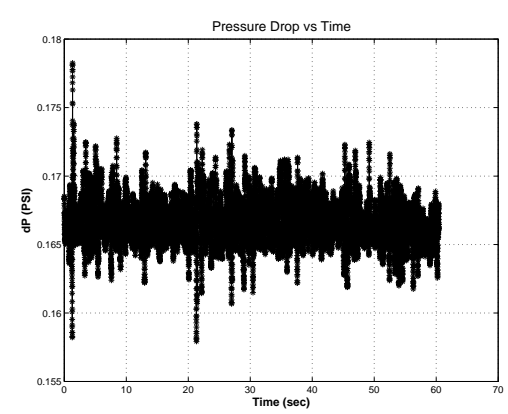

(c) $4 \mathrm{SCFH}$

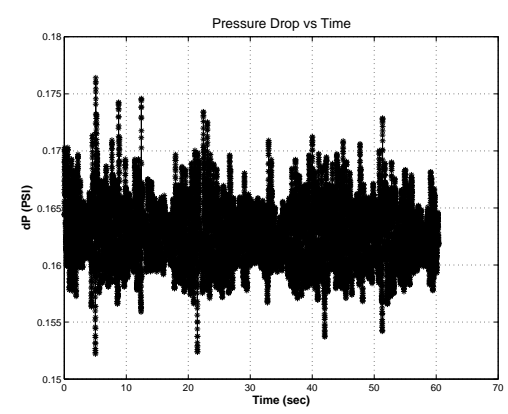

(e) $6 \mathrm{SCFH}$

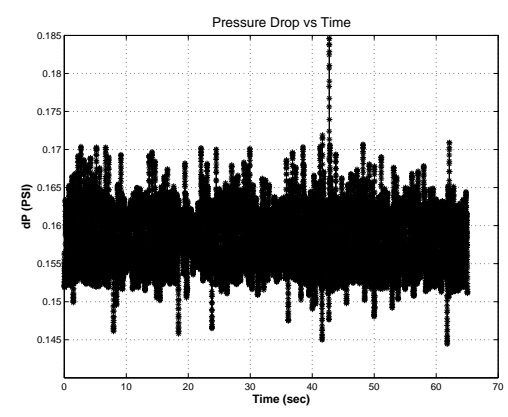

(g) $8 \mathrm{SCFH}$

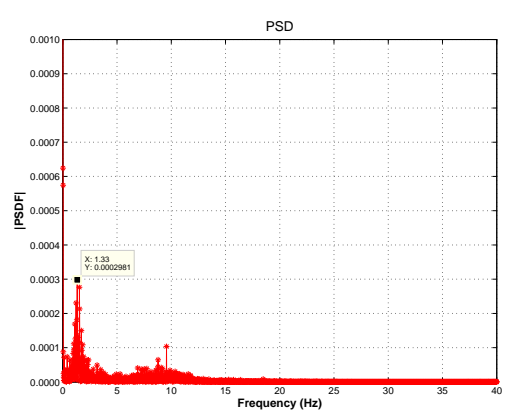

(b) $2 \mathrm{SCFH}$

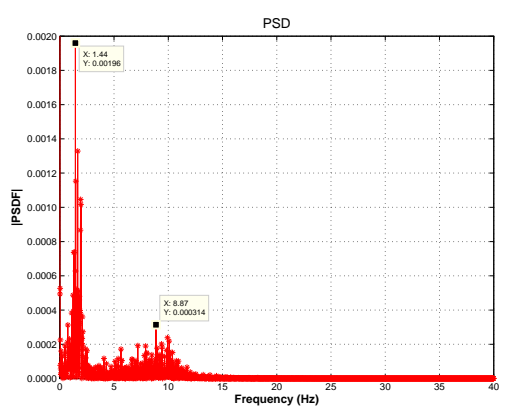

(d) $4 \mathrm{SCFH}$

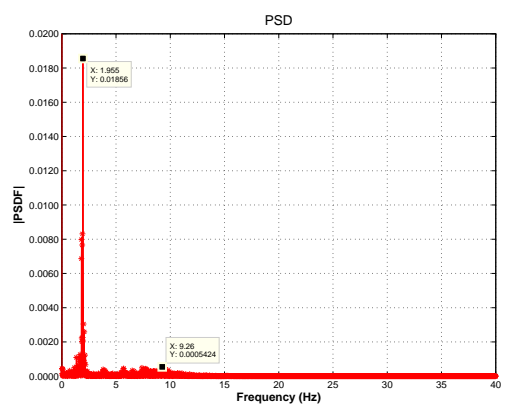

(f) $6 \mathrm{SCFH}$

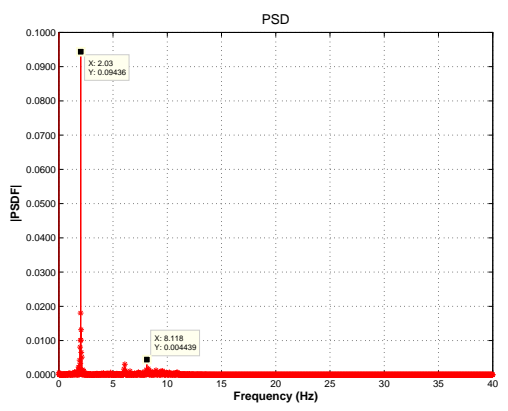

(h) $8 \mathrm{SCFH}$

Figure 4.16: Analysis of the differential pressure signal and its corresponding power spectral density [2-8 SCFH] 


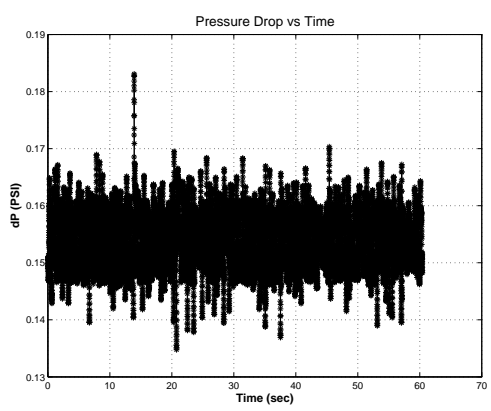

(i) $10 \mathrm{SCFH}$

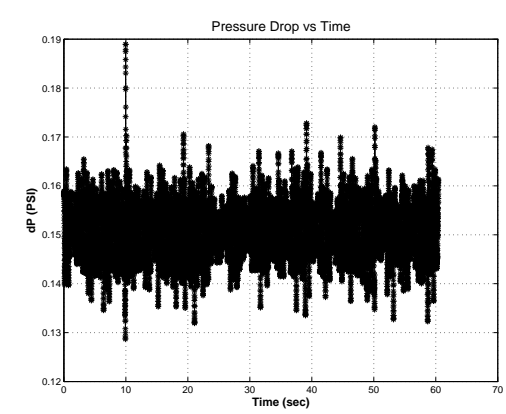

(k) $12 \mathrm{SCFH}$

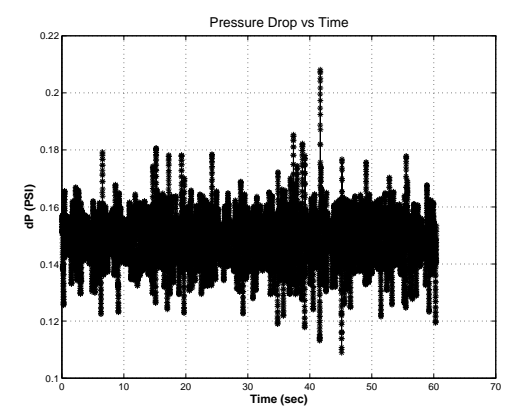

(m) $14 \mathrm{SCFH}$

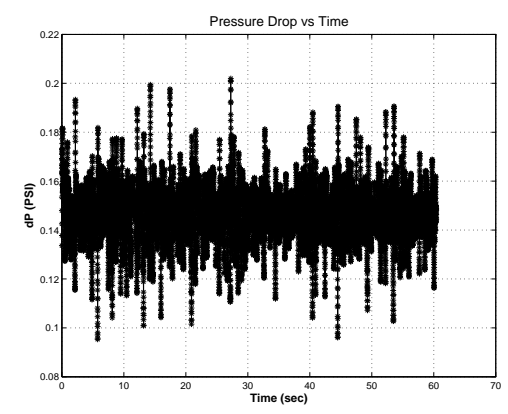

(o) $16 \mathrm{SCFH}$

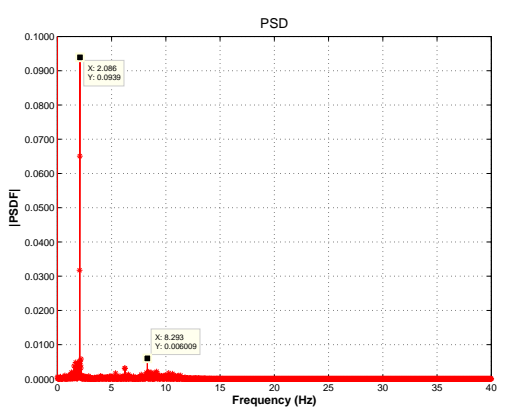

(j) $10 \mathrm{SCFH}$

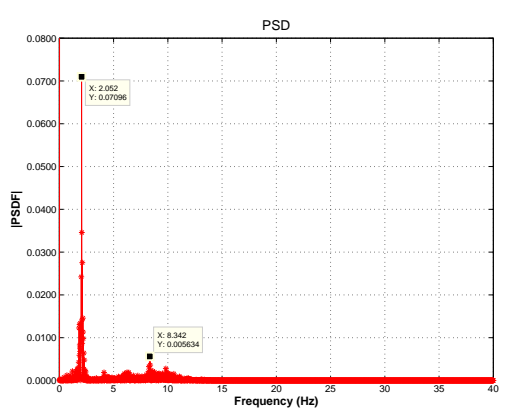

(1) $12 \mathrm{SCFH}$

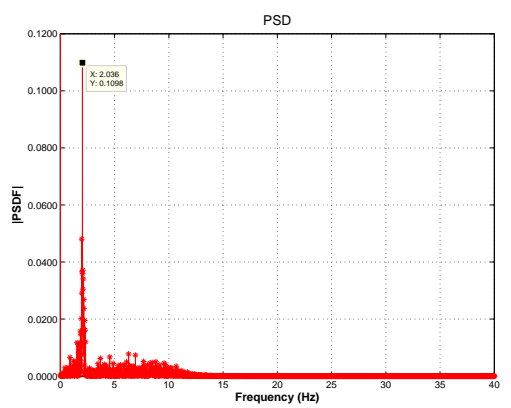

(n) $14 \mathrm{SCFH}$

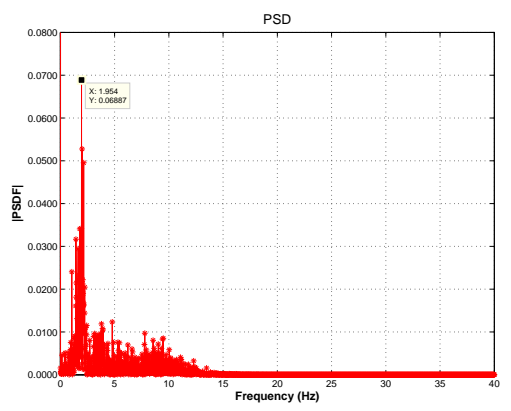

(p) $16 \mathrm{SCFH}$

Figure 4.16: (Continued) Analysis of the differential pressure signal and the power spectral density[10-16 SCFH] 86 


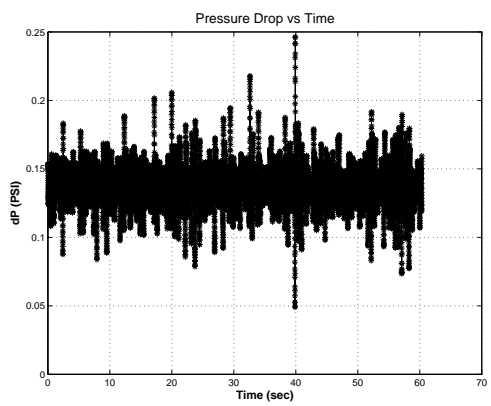

(q) $20 \mathrm{SCFH}$

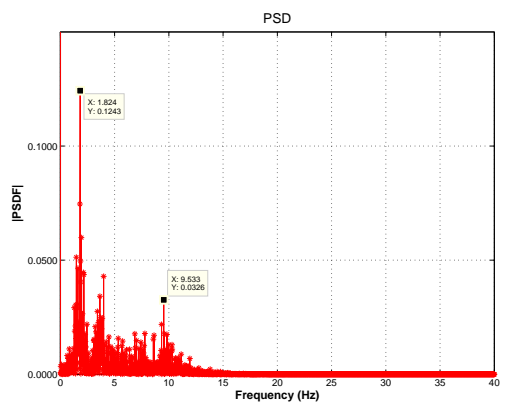

(r) $20 \mathrm{SCFH}$

Figure 4.16: (Concluded) Analysis of the differential pressure signal and its corresponding power spectral density[20 SCFH]

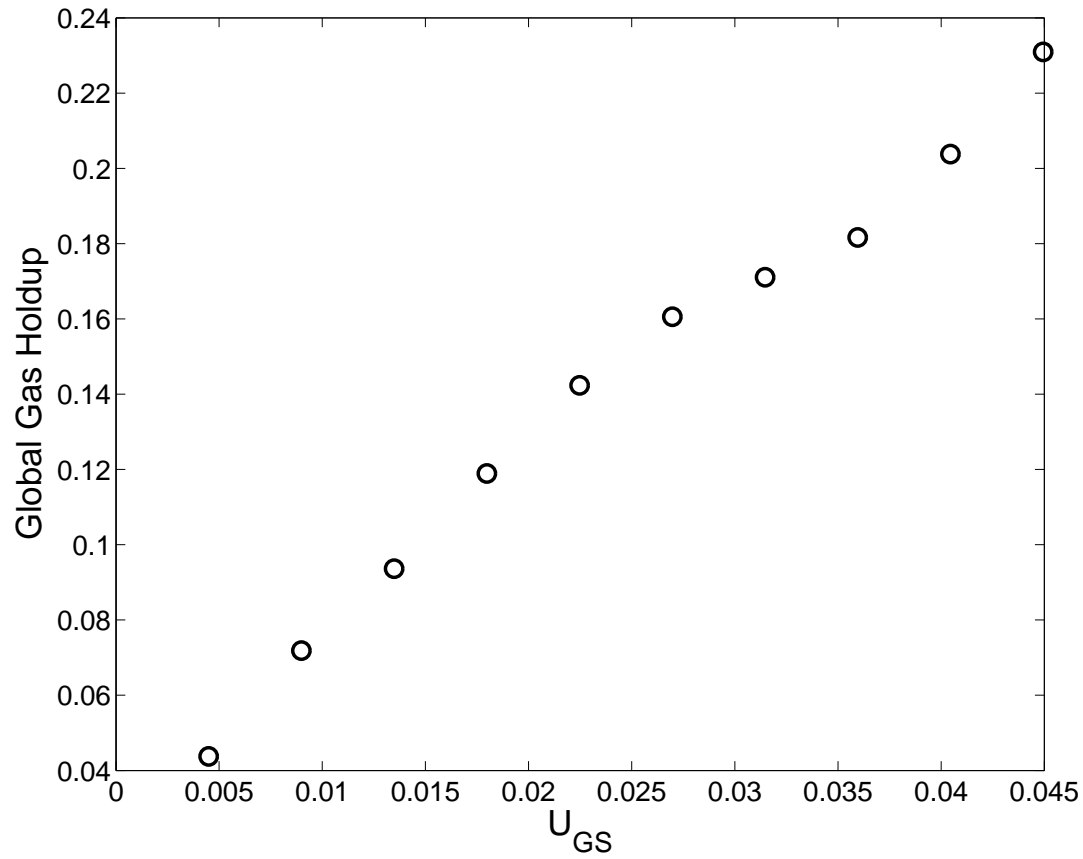

Figure 4.17: Variation of Global Gas Holdup with $U_{G S}(\mathrm{~m} / \mathrm{sec})$ 


\section{Regime Mapping}
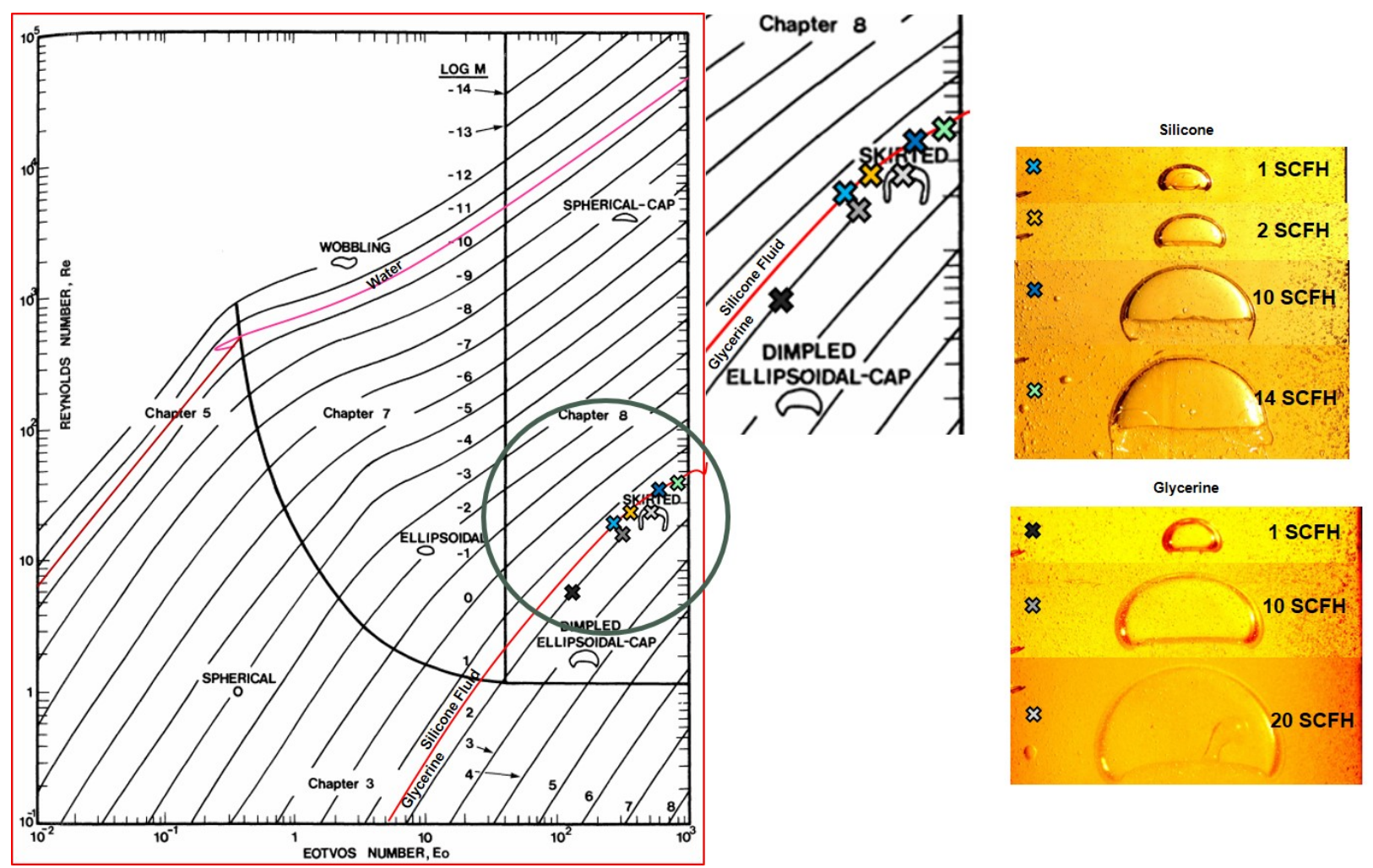

Figure 4.18: Regime mapping on the chart by (Clift et al. , 1988) based on equivalent diameter (de) for Silicone Fluid and Glycerin.

Shown in the Fig. 4.18 is an attempt to arrive at some non-dimensional groups to better characterize the flow, and by extension the shape regimes for the bubbles. The length scale used in the calculation of the Eotvos number is the volume equivalent diameter (de). This is arrived via image processing using imageJ, which assists in the calculation of the projected area. An assumption is then made about the volume of the bubble; as the depth of the bubble column is relatively small $(2 \mathrm{~cm})$ compared to the other dimensions, the bubbles is considered an extruded object (a cylinder) depth wise. It must be asserted, that such as assumption holds itself true only in the case large bubbles which were seen to take up the entire depth of the column available to them; for smaller bubbles the assumption of a rotated object (sphere) could be a fairer approximation. An additional fluid, Glycerin is also studied to draw a comparison with the Silicone Fluid. For the Silicone 
fluid, bubbles arising out of flow rates of 1,2,10 and 14 SCFH have been studied. Visuals show that the 1,2 SCFH cases exhibit a "Dimpled Ellipsoidal-Cap" shape, in the chart the mapped region seems to be lying just outside the skirted region. Going further, the 10,14 SCFH show a skirted structure and their mapping seems to fall inside the skirted regime region on the chart. For Glycerin, the $1 \mathrm{SCFH}$ bubble seems to be well inside the dimpled regime, and is well placed on the chart. The 10, 20 SCFH cases seem on the verge of the skirting are shown to be on the border of the skirted regime on the chart. This exercise, apart from being instructive in arriving at non-dimensional groups to characterize a bubble regime can also be used to arrive at a characteristic Reynolds number, which is defined in terms of the terminal velocity and equivalent diameter. For similar flow rates the Reynolds number for Glycerin is found to be lower than that of the Silicone fluid, and an experiment done with water would have reported higher Reynolds number as mapping would have been along the line of $\log M=-10.5$ as shown in the figure. It must be noted that the Eo number has a significant dependence on the bubble diameter, which in turn could be said to be a function of the mass flow rate of the secondary phase for a given fluid. Therefore for a given fluid, with an increase in inlet velocity, would result in an increase in Eo number whilst climbing along the constant $\log M$ line ; and eventually showcasing an increase in Re due to the change in the length scale (volume equivalent bubble diameter). 


\section{Chapter 5}

\section{Conclusions and Future Work}

\subsubsection{Conclusions}

In this study, the efficacy of two multi-phase numerical models is studied, the Eulerian-Eulerian approach using the commercial software Ansys-Fluent, and EulerianLagrangian approach using an in house LES based Navier-Stokes solver (DREAMII). Both of these approaches use the finite volume method. The assessment is based on the simulation of laboratory scale circulating bubble column.

The plume oscillation period, is found to be fairly well predicted by both the methods for air in water flows. In the absence of a poly dispersed approach, it is found that using smaller bubbles at high velocities gave better agreement with experiments for the E-L approach.

The DREAM code has been further validated across a spectrum of velocities, and the oscillation period thus predicted showed a drop with increase in velocities, as was expected from the published experimental data.

The E-L approach has been found to be able to handle liquids of higher viscosity, with a positive response to increases in velocity showing an increase in the gas hold up of the system. Albeit, with an increase in the computation time, as the number of particle tracks increased with viscoisty

In the case of the E-E modeling approach, the drag law used was found to be very crucial in the accurate prediction of the unsteadiness associated with the bubble plume. 
It has been found that both the E-E and the E-L approach handle the dispersed cases reasonably well. A flow could be termed dispersed if, $l>>D$ where ' $l$ ' is the length scale of the inter grid spacing and 'd' is the interfacial length scale i.e the diameter of the bubble. Although under such consideration the actual bubbles remain unresolved, the overall behaviour of the gas in the liquid flow is well preserved. This essentially springs from the continuum assumption of E-E method, stays relatively true for such flows as the bubbles are closely packed and sometimes interpenetrating with the fluid. For the E-L approach, the discrete and small size of the bubbles is an inherent advantage, as the bubbles could then be treated as particles with point volume without a considerable departure from the true physics.

For higher viscosities, a preliminary investigation shows that the E-L approach (The Dream-II code) does respond to the change in viscosity; but these results are not conclusive yet. Primarily, this is due to inability to accommodate bubbles of 'higher' diameter, that come with fluids of higher viscosity and surface tensions.

The continuum assumption for the E-E method, and the unexplored upper limit for the bubble size in the case of the E-L approach, are the two primary handicaps of the two approaches in handling very viscous media.

Experiments are conducted to better understand the hydrodynamics of the bubbles in very viscous fluids. The big bubbles are found to be caught in each other's wake and accelerate to meet their coalescing partner, especially as the upper bubble approaches the free surface.

The distribution of the smaller bubbles is seen to increase with the increase in the flow rate, and that is reflected in variance of the harmonic mean diameter. A design consideration, that would operate in this regime where the break up phenomena is dominant would be beneficial in obtained better mixing.

Mixing in the viscous experiments is also found to be well characterizing in the sprouting up of new set of higher frequencies in the, power spectral analysis plots of the pressure time series signal. 


\subsubsection{Future Work}

- The inclusion of surface tension as a parameter is conspicuously absent in the models tried thus far; its inclusion is thus essential, for the free surface to play an active role. Inclusion of surface tension, would also avoid the enforcement of the bubble diameter, and thus making it a part of the solution than a part of the problem definition. However, this will require tracking of large bubbles and a bubble breakup and coalescence model.

- One such approach is the Eulerian Volume of Fluid (VOF) approach. In this approach the tracking of the interface happens through the solution of the volume fraction equation, with the fluids not being interpenetrate. Which means the volume fraction of a phase in a cell could only be one or zero, if it lies between zero and 1 then it must contain the interphase with the primary fluid.Such a formulation would be ideal to, solve for the parameters such as the nature and size of the bubbles, a step forward from enforcing them; and thus allowing surface tension forces to play an active role.

- VOF uses interface sharpening schemes, and hence tracking interfaces of the order of the small bubbles could come with a high computational expense. As the VOF method is expected to take care of the large bubbles, the smaller bubbles could be still tracked through a Lagrangian approach. Therefore, it could be a good idea to develop such a hybrid approach and implement it in the DREAM-II solver.

- Some preliminary trials have shown that VOF approach along with adaptive meshing, is very demanding in terms of computational time; therefore the above approach could have more applicability.

- The experiments that were performed with the silicone fluid, should be studied for higher flow rates while measuring velocity changes alongside the pressure reading. It would also be instructive to perform similar experiments 
with lower viscosity fluids, like water; to study the variation of the Harmonic and Sauter diameter, and then a comparative study done. 


\section{Bibliography}

1978. Bubbles, drops and particles. New York, USA: Academic Press.

ANSYS-FLUENT. Fluent Theory Guide.

Basset, A B. 1888. A treatise on hydrodynamics: with numerous examples. Vol. 2. Deighton, Bell and Company.

Becker, S., Sokolichin, A, \& Eigenberger, G. 1994. Gas-liquid flow in bubble columns and loop reactors: Part II. Comparison of detailed experiments and flow simulations. Chemical Engineering Science, 49, 5747-5762.

Becker, S., Bie, H. De, \& Sweeney, J. 1999. Dynamic flow behaviour in bubble columns. Chemical Engineering Science, 54(21), 4929 - 4935.

Beerkens, R. Modular Melting: Industrial glass melting process analysis Part 2. American Ceramic Scoiety Bulletin, 83(7), 35-38.

Beerkens, R. 2004. Modular Melting: Industrial glass melting process analysis Part 1. American Ceramic Scoiety Bulletin, 83(4), 28-32.

Boussinesq, J. 1885. Application des potentiels à l'étude de l'équilibre et du mouvement des solides élastiques: principalement au calcul des déformations et des pressions que produisent, dans ces solides, des efforts quelconques exercés sur une petite partie de leur surface ou de leur intérieur: mémoire suivi de notes étendues sur divers points de physique, mathematique et d'analyse. GauthierVillars.

Buwa, V V, \& Ranade, V V. 2004. Characterization of dynamics of gas-liquid flows in rectangular bubble columns. AIChE journal, 50(10), 2394-2407. 
Camarasa, E, Vial, C, Poncin, S, Wild, G, Midoux, N, \& Bouillard, J. 1999. Influence of coalescence behaviour of the liquid and of gas sparging on hydrodynamics and bubble characteristics in a bubble column. Chemical Engineering and Processing: Process Intensification, 38(46), 329 - 344.

Clift, R, Grace, JR, \& Weber, ME. 1988. Bubbles, Drops and Particles, Academic Press, New York, 1978. Vol. 5. Nos. 1-4, 1988 Modelling of Three Phase Sparged Catalytic Reactors.

Crowe, CT, Sommerfeld, M, \& Tsuji, Y. 1998. Multiphase flows with droplets and particles. CRC Press (Boca Raton, Fla.).

Darmana, D, Henket, RLB, Deen, NG, \& Kuipers, JAM. 2007. Detailed modelling of hydrodynamics, mass transfer and chemical reactions in a bubble column using a discrete bubble model: Chemisorption of $\mathrm{CO} 2$ into $\mathrm{NaOH}$ solution, numerical and experimental study. Chemical engineering science, 62(9), 25562575 .

Delnoij, E., Lammers, F.A., Kuipers, J.A.M., \& van Swaaij, W.P.M. 1997a. Dynamic simulation of dispersed gas-liquid two-phase flow using a discrete bubble model. Chemical Engineering Science, 52, 1429-1458.

Delnoij, E., Kuipers, J.A.M., \& van Swaaij, W.P.M. 1997b. Dynamic simulation of gas-liquid two-phase flow: effect of column aspect ratio on the flow structure. Chemical Engineering Science, 52(21?22), 3759 - 3772.

Díaz, M E, Montes, F J, \& Galán, M A. 2008. Experimental study of the transition between unsteady flow regimes in a partially aerated two-dimensional bubble column. Chemical Engineering and Processing: Process Intensification, 47(9), 1867-1876.

Diaz, M. E, Iranzo, A, Cuadra, D, Barbero, R, J.Montes, F, \& Galan, M A. 2008. Numerical simulation of the gas-liquid flow in a laboratory scale bubble column Influence of bubble size distribution and non-drag forces. Chemical Engineering Journal, 139, 263-379.

Drew, DA, \& Lahey, RT. 1993. Analytical modeling of multiphase flow. Particulate two-phase flow, 509-566. 
Grace, J R, Wairegi, T, \& Nguyen, T.H. 1976. Shapes and velocties of single drops and bubbles moving freely through immiscible liquids. 54a, 167-173.

Hu, G. 2005. Towards large eddy simulation of dispersed gas-liquid two-phase turbulent flows. Ph.D. thesis, West Virginia University.

Hu, G, \& Celik, I. 2008. Eulerian-Lagrangian based large-eddy simulation of a partially aerated flat bubble column. Chemical Engineering Science, 63(1), 253-271.

Kim, J, \& Moin, P. 1985. Application of a fractional-step method to incompressible Navier-Stokes equations. Journal of computational physics, 59(2), 308-323.

Landau, L.D, \& Lifschitz, E.M. 1971. Lehrbuch der Theoretischen Physik. Vol. VI.

Launder, B E, \& Spalding, D B. 1972. Lectures in mathematical models of turbulence.

Lee, M, Malaya, N, \& Moser, R D. 2013. Petascale direct numerical simulation of turbulent channel flow on up to 786K cores. Page 61 of: Proceedings of SC13: International Conference for High Performance Computing, Networking, Storage and Analysis. ACM.

Mathieu, J, \& Scott, J. 2000. An introduction to turbulent flow. Cambridge University Press.

Michaelides, Efstathios E. 2006. Particles, bubbles $\&$ drops: their motion, heat and mass transfer. World Scientific.

Moin, P, \& Kim, J. 1982. Numerical investigation of turbulent channel flow. Journal of fluid mechanics, 118, 341-377.

Mudde, R F, \& Simonin, O. 1999. Two-and three-dimensional simulations of a bubble plume using a two-fluid model. Chemical Engineering Science, 54(21), 5061-5069.

Oey, RS, Mudde, RF, \& Van den Akker, HEA. 2003. Sensitivity study on interfacial closure laws in two-fluid bubbly flow simulations. AIChE journal, 49(7), 16211636 . 
Olmos, E, Gentric, C, Vial, Ch, Wild, G, \& Midoux, N. 2001. Numerical simulation of multiphase flow in bubble column reactors. Influence of bubble coalescence and break-up. Chemical Engineering Science, 56(2122), 6359 - 6365.

Patankar, S V, \& Spalding, D B. 1972. A calculation procedure for heat, mass and momentum transfer in three-dimensional parabolic flows. International Journal of Heat and Mass Transfer, 15(10), 1787-1806.

Pfleger, D, Gomes, S, Gilbert, N, \& Wagner, H-G. 1999. Hydrodynamic simulations of laboratory scale bubble columns fundamental studies of the Eulerian?Eulerian modelling approach. Chemical Engineering Science, 54(21), 5091 -5099 .

Rasband, W S. 1997. ImageJ, US National Institutes of Health, Bethesda, Maryland, USA.

Ravinuthala, S C, \& Celik, I. 2013. Hydrodynamic causes and effects of air bubbles rising in very viscous media. arXiv preprint arXiv:1310.2998.

Rightley, Paul M. 1995. Bubble dispersion and interphase coupling in a free shear flow. University of California, San Diego.

Ross, C. Philip, \& Tincher, Gabe L. 2004. Glass Melting Technology: A technical Assesment. U.S Department of Energy.

Rue, D, \& Brown, J T. 2011. Submerged Combustion Melting of Glass. International Journal of Applied Glass Science, 2(4), 262-274.

Ruthiya, K C, Chilekar, V P, Warnier, Maurice JF, van der Schaaf, J, Kuster, Ben FM, Schouten, J C, \& van Ommen, J R. 2005. Detecting regime transitions in slurry bubble columns using pressure time series. AIChE journal, 51(7), 1951-1965.

Schiller, L, \& Naumann, A. 1935. A drag coefficient correlation. Vdi Zeitung, 77, 318-320.

Schlichting, H, \& Gersten, K. 2000. Boundary-layer theory. Springer. 
Shah, Y. K, Godbole, S., \& Deckwer, W. 1982. Design parameters estimations for bubble column reactors. AICHE journal, 28(3), 353-379.

Smagorinsky, J. 1963. GENERAL CIRCULATION EXPERIMENTS WITH THE PRIMITIVE EQUATIONS: I. THE BASIC EXPERIMENT*. Monthly weather review, 91(3), 99-164.

Sokolichin, A, \& Eigenberger, G. 1999. Applicability of the standard k-epsilon turbulence model to the dynamic simulation of bubble columns: Part 1. Detailed numerical simulations. Chemical Engineering Science, 54, 2273-2284.

Studley, Allison F. 2010. Numerical Modeling of Air-Water Flows in Bubble Columns and Airlift Reactors. Ph.D. thesis, Virginia Polytechnical Institute and State University.

Tzeng, J-W, Chen, RC, \& Fan, L-S. 1993. Visualization of flow characteristics in a 2-D bubble column and three-phase fluidized bed. AIChE journal, 39(5), $733-744$.

V.Buwa, V, \& V.Ranade, V. 2002. Dynamics of gas-liquid flow in a rectangular bubble column: experiments and single/multi-group CFD simulations. Chemical Engineering Science, 57, 4715-4736.

White, FM. 2006. Viscous fluid flow. McGraw-Hill series in mechanical engineering. McGraw-Hill Higher Education New York. 


\section{Appendix A User Defined Function for the Clift - Grace Drag Law}

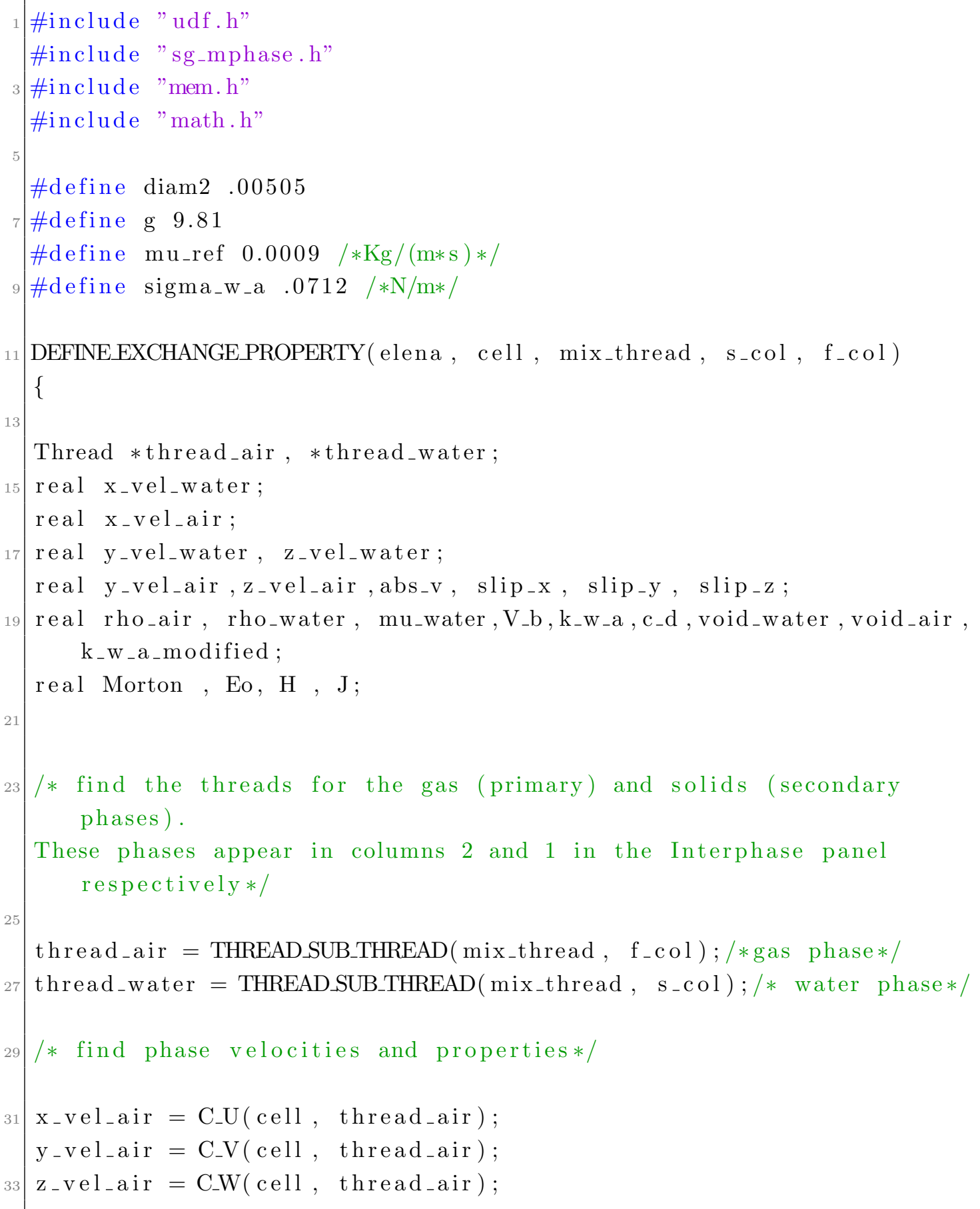




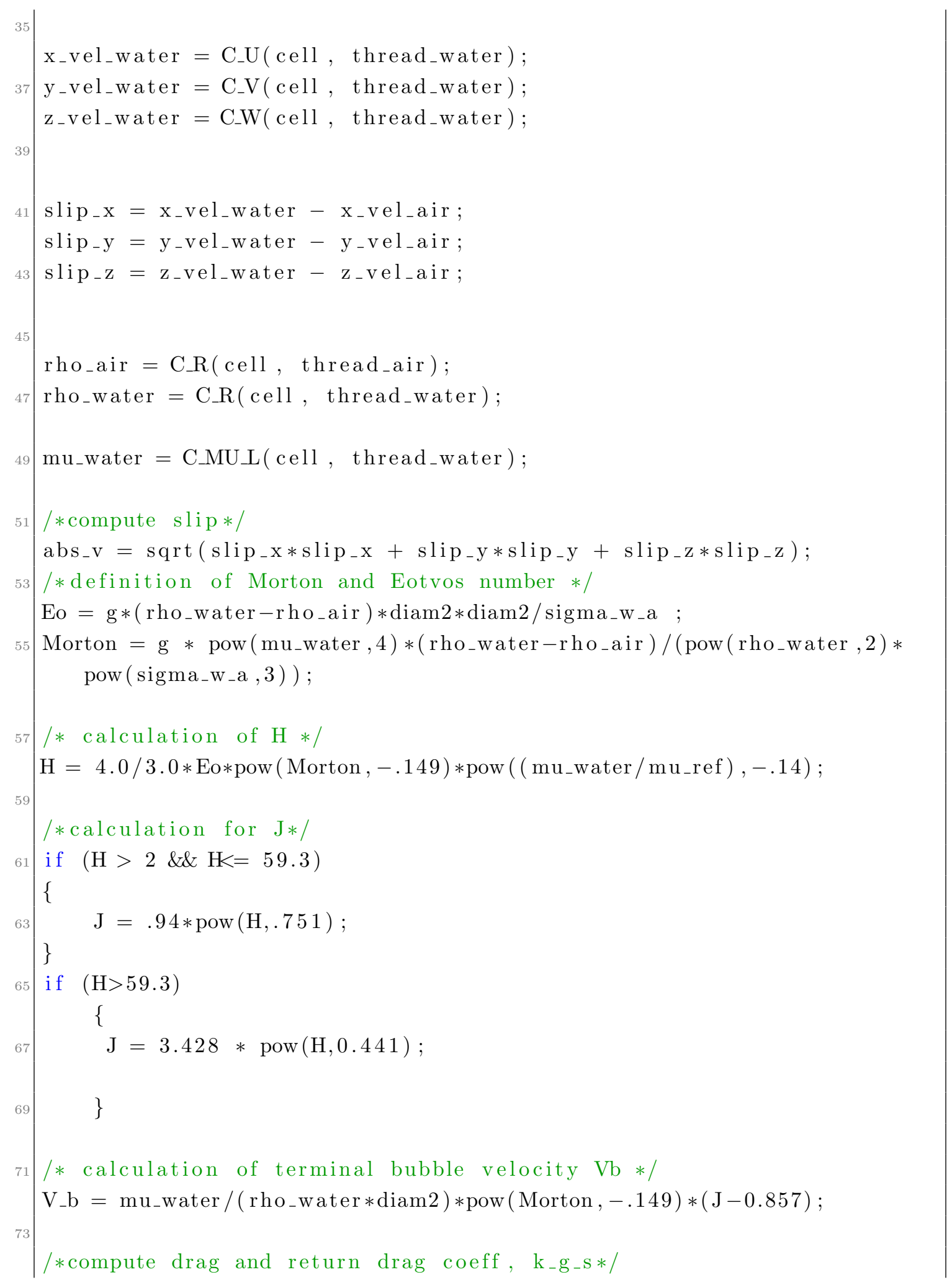




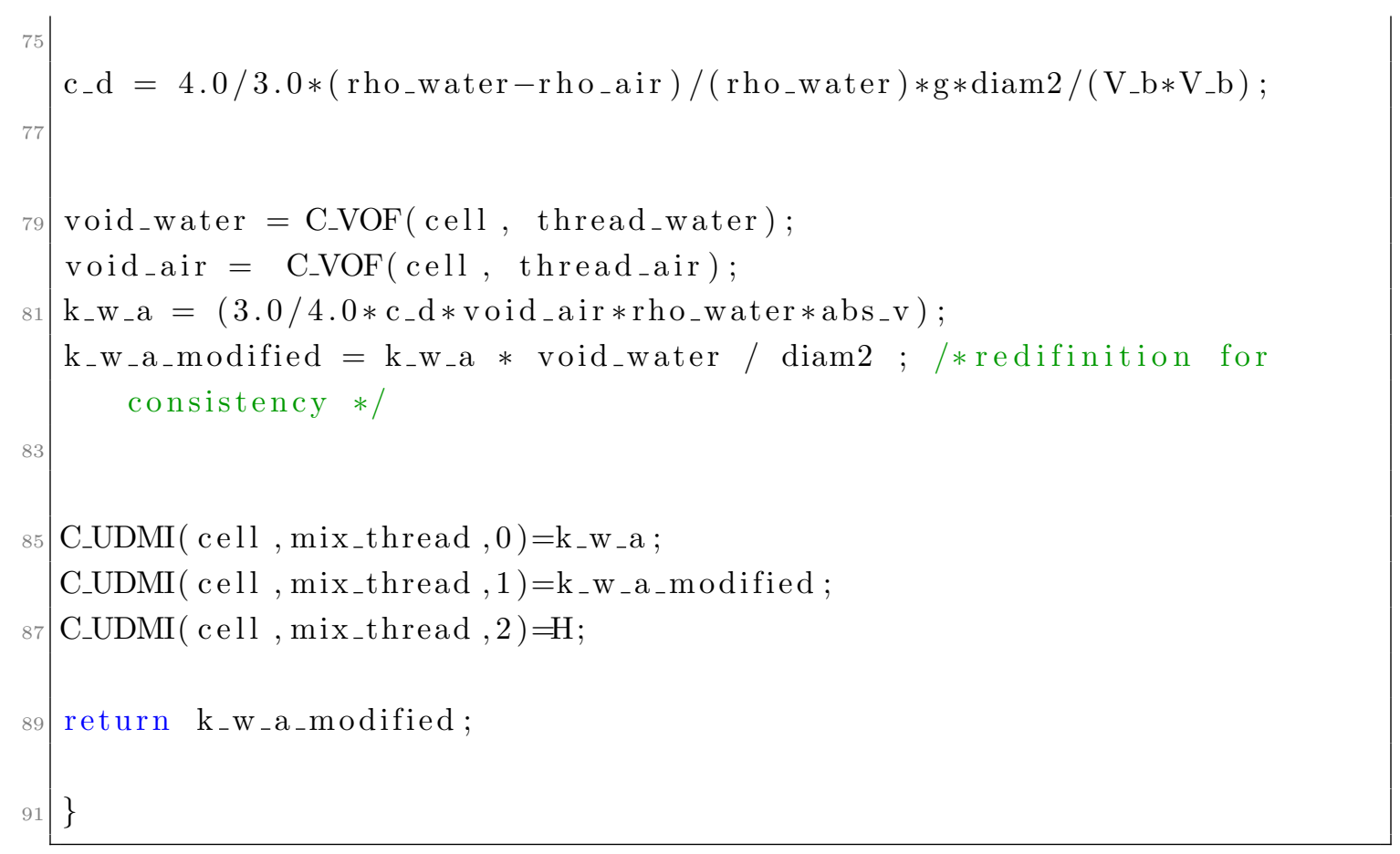




\section{Appendix B Bubble Size Distribution in Physical Units (cm)}

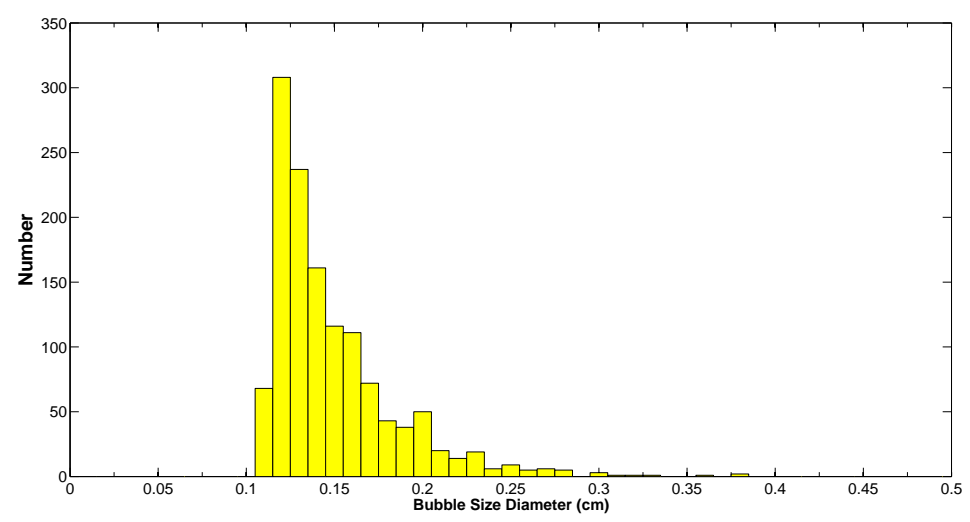

(a) $1 \mathrm{SCFH}$

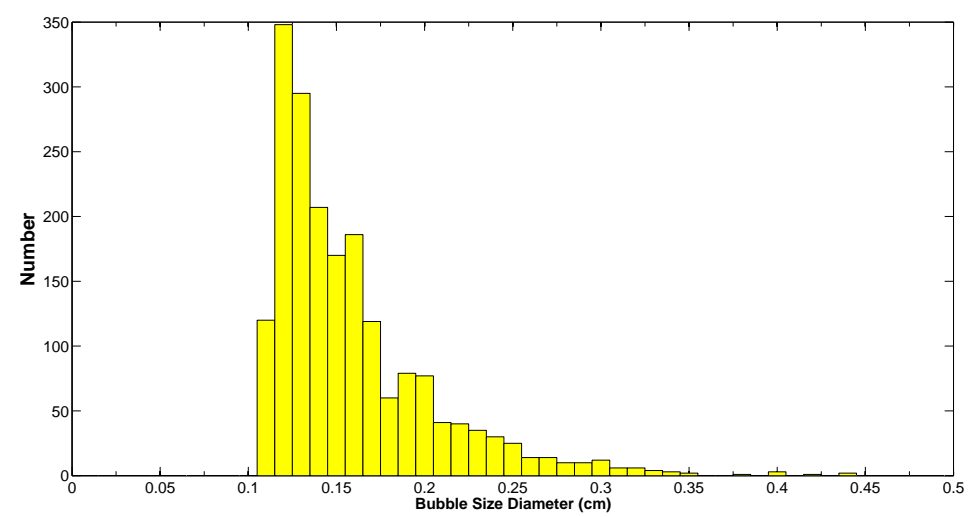

(b) $2 \mathrm{SCFH}$

Figure 1: Particle size (in $\mathrm{cm}$ ) distribution for[1-2 SCFH] from the image analysis using ImageJ. 


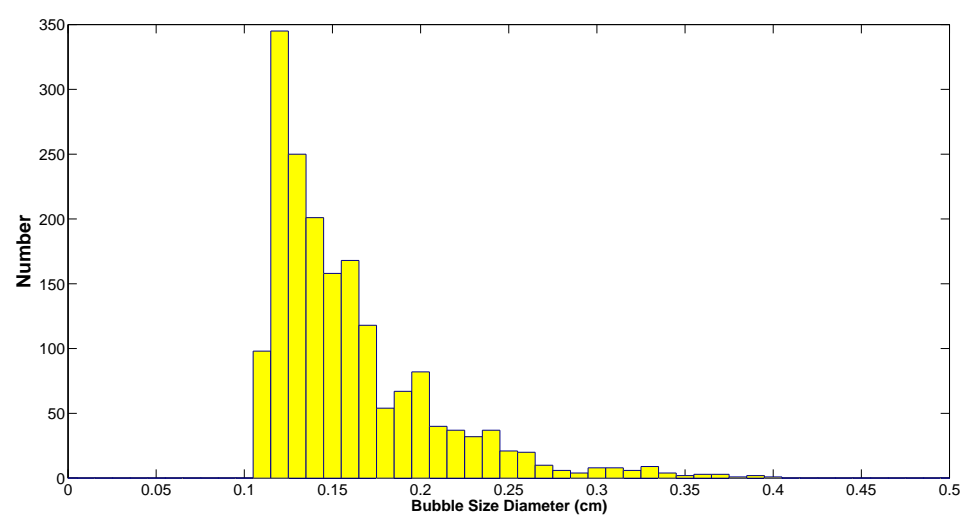

(c) $6 \mathrm{SCFH}$

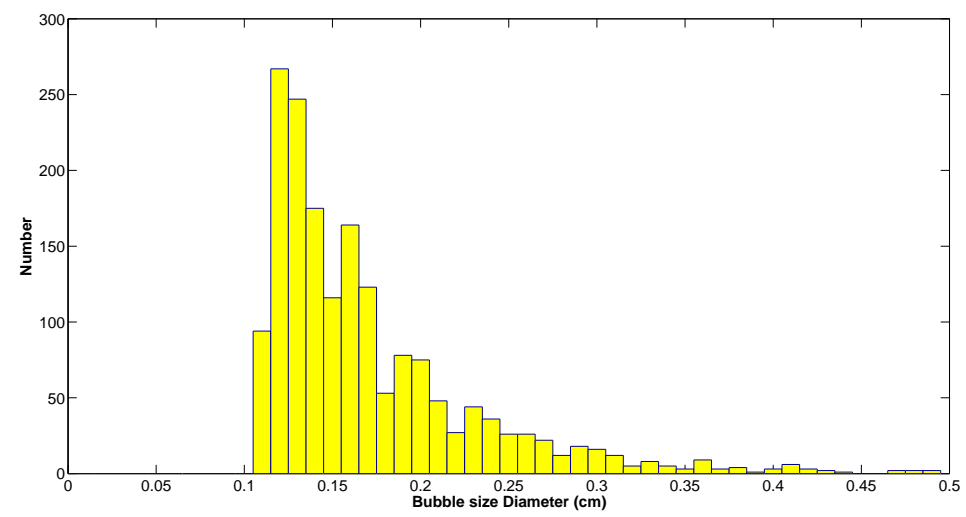

(d) $8 \mathrm{SCFH}$

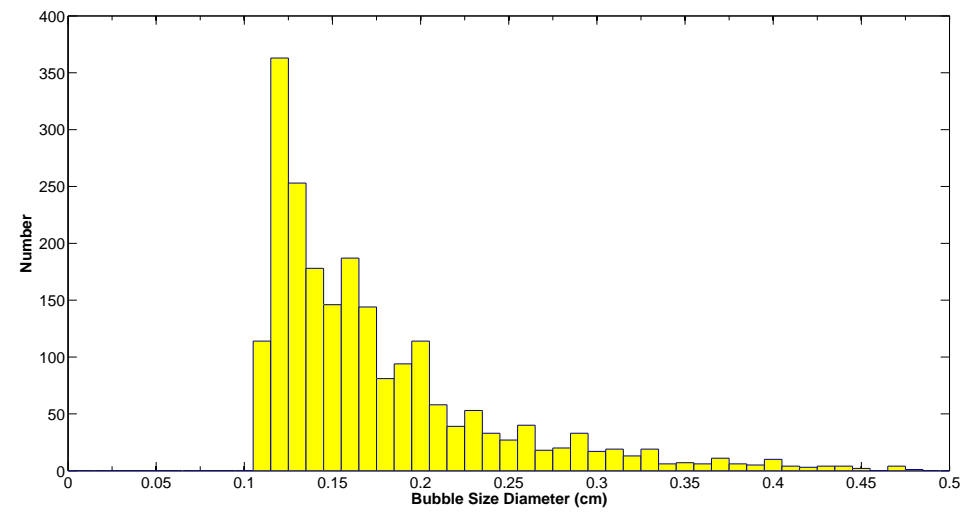

(e) $10 \mathrm{SCFH}$

Figure 1: Particle size (in $\mathrm{cm}$ ) distribution for [6-10 SCFH] from the image analysis using ImageJ. 


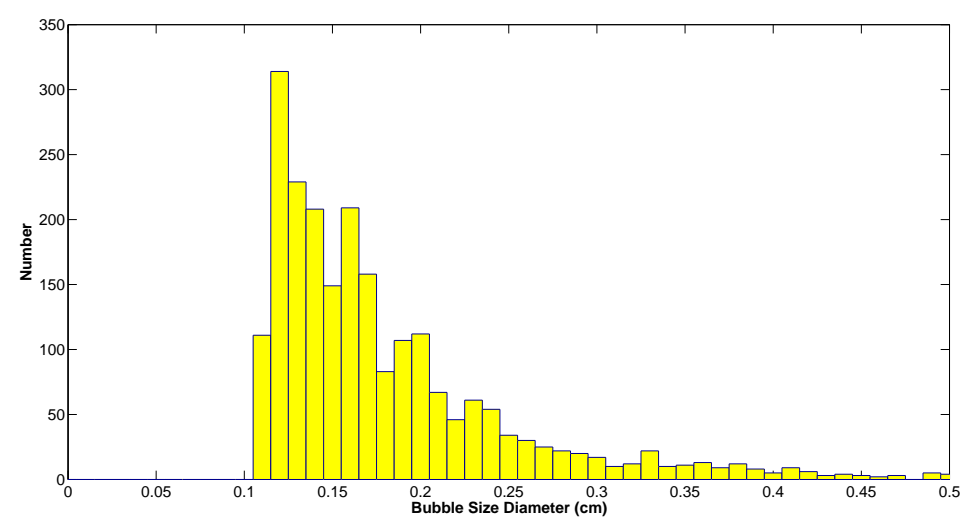

(f) $11 \mathrm{SCFH}$

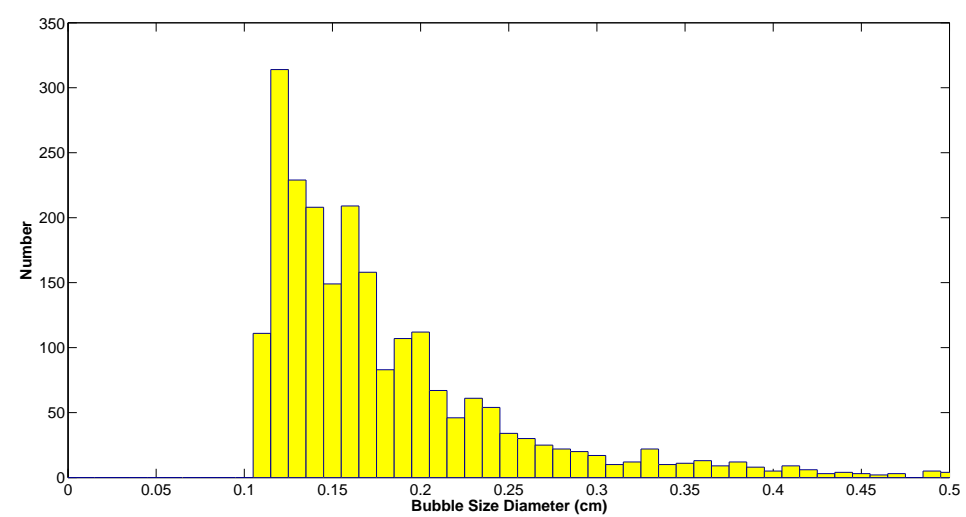

(g) $16 \mathrm{SCFH}$

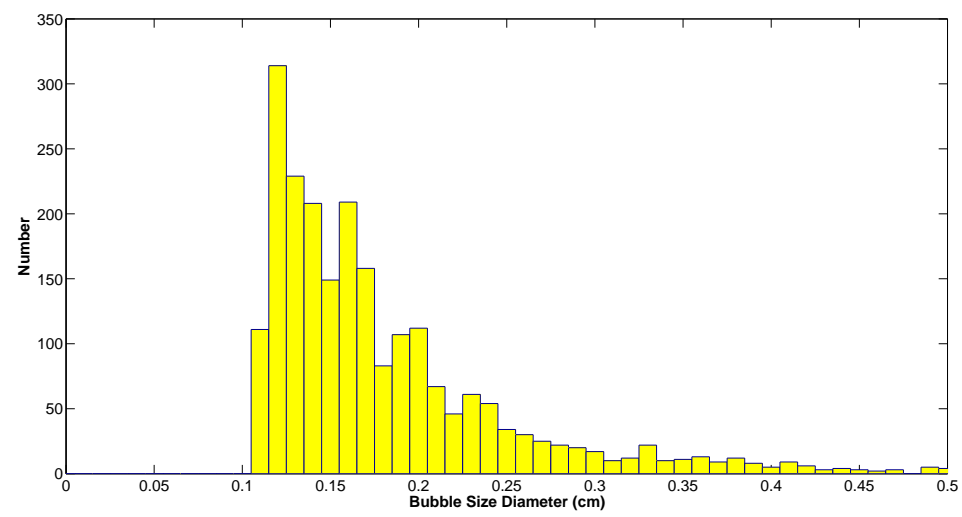

(h) $18 \mathrm{SCFH}$

Figure 1: (Concluded)Particle size (in $\mathrm{cm}$ ) distribution for[11-18 $\mathrm{SCFH}]$ from the image analysis using ImageJ. 